\title{
Laboratory Directed Research and Development Program FY 2004
}

March 2005 


\section{DISCLAIMER}

This document was prepared as an account of work sponsored by the United States Government. While this document is believed to contain correct information, neither the United States Government nor any agency thereof, nor The Regents of the University of California, nor any of their employees, makes any warranty, express or implied, or assumes any legal responsibility for the accuracy, completeness, or usefulness of any information, apparatus, product, or process disclosed, or represents that its use would not infringe privately owned rights. Reference herein to any specific commercial product, process, or service by its trade name, trademark, manufacturer, or otherwise, does not necessarily constitute or imply its endorsement, recommendation, or favoring by the United States Government or any agency thereof, or The Regents of the University of California. The views and opinions of authors expressed herein do not necessarily state or reflect those of the United States Government or any agency thereof or The Regents of the University of California.

Lawrence Berkeley Laboratory is an equal opportunity employer. 


\section{Report on \\ Ernest Orlando Lawrence Berkeley National Laboratory}

\section{Laboratory Directed Research and Development Program}

FY 2004

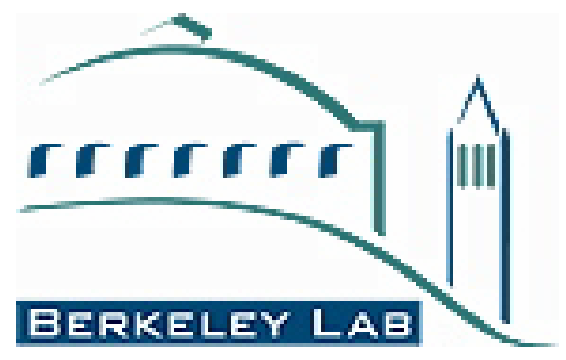

Ernest Orlando Lawrence Berkeley National Laboratory Berkeley, CA 94720
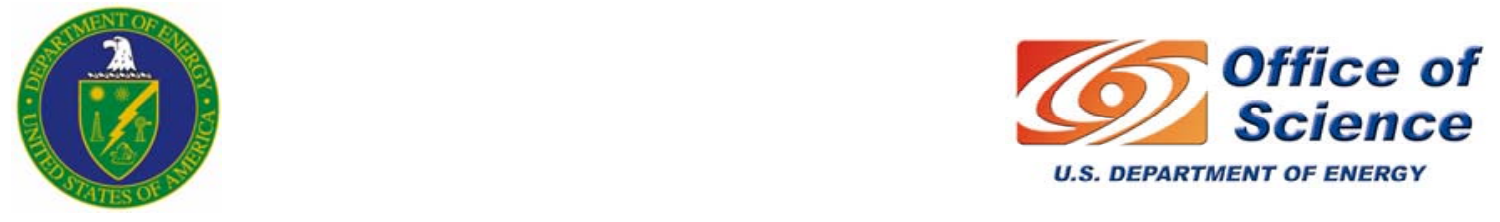

Prepared for the U.S. Department of Energy under Contract No. DE-AC03-76SF00098 



\section{Table of Contents}

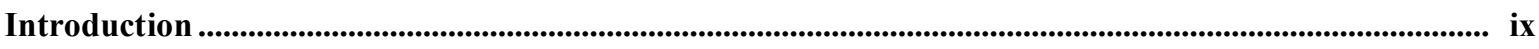

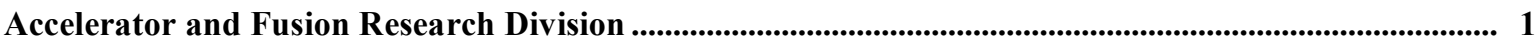

Daniel Dietderich

Soren Prestemon

William Fawley

Wim Leemans

Ka-Ngo Leung

Raul Richardson

Steven Lidia

Alexander Zholents

John Corlett

Stephen Leone

Howard Padmore

Robert Schoenlein

Russell Wells

Esmond $\mathrm{Ng}$

Robert Ryne

GianLuca Sabbi

Thomas Schenkel

Jeffrey Bokor

Miguel Furman

Jean-Luc Vay
Short Period Superconducting Undulator Development.

Analysis and Modeling of Multicore Induction Cell Voltage

Distribution

Novel Coherent THz and IR Source Using a Laser Wakefield

Accelerator and Applications

Development of a DNA/RNA Sequencing Tool

4

Critical Accelerator Technologies for Future Advanced Light

Sources .

Optimal Solvers for Infinite-Dimensional Hamiltonian Systems ..

6

Superconducting Magnet Systems for Ex Situ NMR

Spectroscopy

7

Solid State Quantum Computer Development with Single Ion Implantation .

Electron Production and Collective Field Generation in Intense Particle Beams

9

Malcolm Howells

Henry Chapman

Chris Jacobsen

Janos Kirz

Howard Padmore

John Spence

Uwe Weierstall

Zahid Hussain

Eric Gullikson

M. Zahid Hasan

Janos Kirz

Michael Martin

John Byrd

Fernando Sannibale

Wayne McKinney

David Robin 
Howard Padmore

David Robin

Ulrich Dahmen

Shaul Mukamel

Robert Schoenlein

Andreas Scholl

Howard Padmore

Joachim Stöhr

Tracy Young

Thornton Glover

Neville Smith

Stephan Kevan

K. Chenel

Jeffrey Kortright

Russell Wilcox

Robert Schoenlein
Thornton Glover

Aberration Correction of Electron Microscopes

14

Simulations of Femtosecond X-ray Spectra of Photoexcited

Molecules

15

Ultrafast Magnetization Dynamics.

16

Developing Dynamic Soft X-ray Scattering to Probe Spatial and

Temporal Fluctuations in Nanomagnets

High Average Power Laser Amplifier

Masahid Ahmed

Gas Phase Studies of the Building Blocks of Life

Corwin Booth

Disorder and Multiple Length Scales in Non-Fermi Liquid f-

electron Intermetallics

Stephen Leone

Coherent Control and Quantum Information in Polyatomic

Molecules

Daniel Neumark

Spectroscopy and Dynamics of Pure and Doped Helium

Nanodroplets

Soft X-ray Spectroscopy of Liquid Surfaces

David Shuh

David Shuh

Scientific Investigations and Technique Development of Wet

Spectroscopy High Pressure Photoelectron Spectroscopy, and

STXM for Molecular Environmental Science

Computing Sciences

(National Energy Research Scientific Computing Center, Computational Research. and Information

Technologies and Services Divisions )

25

David Bailey

Experimental Mathematician’s Toolkit

Xiaoye Li

Silvia Crivelli

E. Wes Bethel

Infrastructure for Improving Protein Structure Prediction in Computational Biology....

Chris Ding

Bernd Hamann

New Machine Learning and Data Mining Methods for Genomics and Climate Data Analysis

Investigative Visualization Methods for Exploration and Comparison of Multi-billion Base Pair Sequence Data

Nancy Meyer

Optimizing Genomic Data Storage for Wide Accessibility

Damian Hazen

Jonathan Carter 
Juan Meza

Ricardo Oliva

Silvia Crivelli

Ali Pinar

Thomas Rescigno

C. William McCurdy

Paul Hargrove

KatherineYelick
Parallel Methods for Robust Optimization and Uncertainty

Quantification

Combinatorial Algorithms in Scientific Computing

31

Advanced Computational Methods for Photon-Molecule

Collision Processes ......................................................................... 32

Evaluation of Computer Architecture Alternatives ........................ 33

Earth Sciences Division

Lisa Alvarez-Cohen

Terry Hazen

Jillian Banfield

James Bishop

William Edwards

Michael Vestel

Gudmundur Bodvarsson

Sharon Borglin

Christopher Campbell

Bailey Green

Hoi-Ying Holman

William Stringfellow

Allen Grayson

Norman Miller

Nigel Quinn

Larry Dale

Katie Coughlin

Helen $\mathrm{He}$

George Moridis

Stefan Finsterle

Eric Sonnenthal

Garrison Sposito

Kurt Nihei

Steven Pride

Ernest Majer

Glenn Waychunas

Jillian Banfield

Hoi-Ying Holman

A. Paul Alivisatos
Application of Real-time PCR with Reverse Transcription for Quantification of Specific Microbial Activity in Complex Communities......

Microbial Controls on Metals in the Environment......................... 35

Autonomous Sensors for Ocean Dissolved Organic Matter.......... 36

Comparative Studies Between Earth and Planetary Science

Development of Biosensors for Endocrine Disrupting

Compounds in Agricultural Watersheds.

The California Water and Energy Systems: An Approach for Addressing Future Crises.

Next Generation Codes for Modeling Subsurface Processes

Advancing the Next Generation of Rock-fluid Imaging and Stimulation Technologies

Reactivity of Nanoparticles in Natural Environments

42

Environmental Energy Technologies Division

Michael Apte

Miniaturized Systems for Particle Exposure Assessment.

Lara Gundel

Anthony Hansen

Thomas Kirchstetter

Determining the Light-Absorbing Properties of Aerosol

Particles 
Nitash Balsara

Kyungoul Baek

Robert Kostecki

Samuel Mao

Richard Russo

Surabi Menon

Jayant Sathaye

Mark Levine

Lynn Price

Stephane de la Rue

du Can

Jonathan Sinton

Ernst Worrell

Nan Zhou

Robert Van Buskirk

Shaheen Tonse
Novel High-Temperature Membranes and Electrocatalysts

Structures for Fuel Cells

Nanostructured Cathodes for Efficient Organic LEDs

46

Evaluating Aerosol Effects on Regional and Global Energy and

Water Budgets

Long Term Global Energy Demand and Carbon Emissions

Scenarios

Evaluation of Dynamic Air Quality Impacts of Distributed

Generation.

Genomics Division.

Eugene Myers

Making the Most of Sequencing: Improved Assembly and Comparative Annotation

Eddy Rubin

Identification and Characterization of Conserved Noncoding

Sequences Using Comparative Genomics and Transgenic

Technology

Life Sciences Division

Manfred Auer

Felicia Betancourt

Robert Glaeser

Mark Biggin

Jian Jin

Ursula Schulze-Gahmen

Priscilla Cooper

Abby Dernburg

Joe Gray

William Jagust

Gary Karpen

G. Steven Martin

Mina Bissell

I. Saira Mian
Molecular Microscopy and Tomography: 3D Visualization, Localization and Quantitative Analysis of Molecular Machines

in Cells and Tissues at Molecular Resolution 52

Membrane-Protein Cryo-electron Microscopy 53

High Throughput Strategy to Identify Protein Complexes 54

Analysis of Complex Phosphorylation Patterns in a Key DNA

Repair Protein by Coupling Surface Plasmon Resonance

Dynamic Reorganization of Chromosome Architecture during Meiosis

Functional Interpretation of Cancer Genomes.

Neuroimaging with Advanced Molecular Probes

Identification and Analysis of Determinants of Centromere Identity in Drosophila ....

Imaging Three-dimensional Signaling Networks in Normal and Malignant Tissue 60

Systems Biology: Biological Input-Output Devices 61 
Carlos Ortiz Characterization of Adult Stem Cell Involvement in Mammary

de Solórzano

Gland Development

Materials Sciences Division

A. Paul Alivisatos

Properties of Nanocrystals Under Extreme Conditions

Molecular Control of Interfaces Between Biological and Synthetic Materials.

Techniques of Sample Controls for a Transmission Electron Aberration-corrected Microscope.

Directed Assembly of Germanium Island Arrays on Gold-

Oscar Dubón

J. Alexander Liddle

Dirk Trauner

Matthew Francis

J. Alexander Liddle

Nitash Balsara

Jeffrey Long

Joel Moore

Yuri Suzuki
Patterned Si(100)

Development of Light-Switchable Potassium Channels

Nanoscale Lithography to Guide Self-assembly for the Creation of Functional, Hierarchical Nanostructures

Extending Electron Delocalization in Mixed-Valent Molecular Assemblies

Modeling Quantum Coherence and Transport in Nanosclae Spin, Charge, and Flux Devices

Magnetism at the Nanometer Scale in Spin Polarized Materials ...
63

Paulo Bedaque

Effective Field Theory and Few-Nucleon Systems. 72

Research and Development of Double Beta Decay Experiments .. 73

Kevin Lesko

Research on a Next Generation Vertex Detector 74

Howard Wieman

Howard Matis

Physical Biosciences Division

Jamie Cate

Thomas Earnest

Sung-Hou Kim

Edward Berry

Kenneth Downing

Robert Glaeser

John Kuriyan

Michael Marletta

Gerry McDermott

Robert Tjian

Haw Yang
Microscopic Imaging in High-Throughput Screening for Crystals of the Bacterial Ribosome 75

High-throughput Production of Proteins and Protein Complexes 76

Structural Genomics Tools for Membrane Proteins. 77

Allosteric Mechanisms in Proteins Involved in Cell Signaling..... 78

Structure and Functional Characterization of Heme Protein Sensors.

Development of Techniques for the Study of Large Macromolecular Complexes Using X-ray Crystallography 80

Conformation and Reaction Dynamics at the Single Molecule Level 81 82 
Stuart Freedman

Karsten Heeger

Richard Kadel

Kam-Biu Luk
Designing a Novel Reactor Neutrino Oscillation Experiment for

Measuring the Unknown Mixing Angle Theta-13

Cross-Divisional .

Phillip Geissler

Microscopic Theory of Protein Surface.

Charles Harris

Investigation of Charge Transfer in Organic Electronics Using

John Arnold

Ulrafast Spectroscopy and Targeted Synthesis 85

John Kerr

Stephen Johnson

Alexander Pines

John Clarke

"Ex-Situ" and "Remote" Molecular Imaging and Spectroscopy...

David Wemmer

Jeffrey Reimer

Steven Gourlay

GianLuca Sabbi

Ernest Majer

Dimitris Budker

Thomas Budinger

Publications List... 


\section{Introduction}

The Ernest Orlando Lawrence Berkeley National Laboratory (Berkeley Lab or LBNL) is a multi-program national research facility operated by the University of California for the Department of Energy (DOE). As an integral element of DOE's National Laboratory System, Berkeley Lab supports DOE's missions in fundamental science, energy resources, and environmental quality. Berkeley Lab programs advance four distinct goals for DOE and the nation:

- To perform leading multidisciplinary research in the computing sciences, physical sciences, energy sciences, biosciences, and general sciences in a manner that ensures employee and public safety and protection of the environment.

- To develop and operate unique national experimental facilities for qualified investigators.

- To educate and train future generations of scientists and engineers to promote national science and education goals.

- To transfer knowledge and technological innovations and to foster productive relationships among Berkeley Lab's research programs, universities, and industry in order to promote national economic competitiveness.

Berkeley Lab's research and the Laboratory Directed Research and Development (LDRD) program support DOE's Strategic Goals that are codified in DOE's September 2003 Strategic Plan, with a primary focus on Advancing Scientific Understanding. For that goal, the Fiscal Year (FY) 2004 LDRD projects support every one of the eight strategies described in the plan. In addition, LDRD efforts support the goals of Investing in America's Energy Future (six of the fourteen strategies), Resolving the Environmental Legacy (four of the eight strategies), and Meeting National Security Challenges (unclassified fundamental research that supports stockpile safety and nonproliferation programs). The LDRD supports Office of Science strategic plans, including the 20 year Scientific Facilities Plan and the draft Office of Science Strategic Plan. The research also supports the strategic directions periodically under review by the Office of Science Program Offices, such as strategic LDRD projects germane to new research facility concepts and new fundamental science directions.

Berkeley Lab LDRD program also play an important role in leveraging DOE capabilities for national needs. The fundamental scientific research and development conducted in the program advances the skills and technologies of importance to our Work For Others (WFO) sponsors.
Among many directions, these include a broad range of health-related science and technology of interest to the National Institutes of Health, breast cancer and accelerator research supported by the Department of Defense, detector technologies that should be useful to the Department of Homeland Security, and particle detection that will be valuable to the Environmental Protection Agency.

The Berkeley Lab Laboratory Directed Research and Development Program FY2004 report is compiled from annual reports submitted by principal investigators following the close of the fiscal year. This report describes the supported projects and summarizes their accomplishments. It constitutes a part of the LDRD program planning and documentation process that includes an annual planning cycle, project selection, implementation, and review.

The Berkeley Lab LDRD program is a critical tool for directing the Laboratory's forefront scientific research capabilities toward vital, excellent, and emerging scientific challenges. The program provides the resources for Berkeley Lab scientists to make rapid and significant contributions to critical national science and technology problems. The LDRD program also advances Berkeley Lab's core competencies, foundations, and scientific capability, and permits exploration of exciting new opportunities. All projects are work in forefront areas of science and technology. Areas eligible for support include the following:

- Advanced study of hypotheses, concepts, or innovative approaches to scientific or technical problems;

- Experiments and analyses directed toward "proof of principle" or early determination of the utility of new scientific ideas, technical concepts, or devices; and

- Conception and preliminary technical analyses of experimental facilities or devices.

The LDRD program supports Berkeley Lab's mission in many ways. First, because LDRD funds can be allocated within a relatively short time frame, Berkeley Lab researchers can support the mission of the Department of Energy (DOE) and serve the needs of the nation by quickly responding to forefront scientific problems. Second, LDRD enables Berkeley Lab to attract and retain highly qualified scientists and to support their efforts to carry out worldleading research. In addition, the LDRD program also supports new projects that involve graduate students and postdoctoral fellows, thus contributing to the education mission of Berkeley Lab. 
Berkeley Lab has a formal process for allocating funds for the LDRD program. The process relies on individual scientific investigators and the scientific leadership of Berkeley Lab to identify opportunities that will contribute to scientific and institutional goals. The process is also designed to maintain compliance with DOE Orders, in particular DOE Order 413.2A, dated January 8, 2001. From year to year, the distribution of funds among the scientific program areas changes. This flexibility optimizes Berkeley Lab's ability to respond to opportunities.

Berkeley Lab LDRD policy and program decisions are the responsibility of the Laboratory Director. The Director has assigned general programmatic oversight responsibility to the Deputy Director. Administration and reporting on the LDRD program is supported by the Directorate's Office for Planning and Strategic Development. LDRD accounting procedures and financial management are consistent with the Laboratory's accounting principles and stipulations under the contract between the University of California and the Department of Energy, with accounting maintained through the Laboratory's Chief Financial Officer.

In FY2004, Berkeley Lab was authorized by DOE to establish a funding ceiling for the LDRD program of $\$ 16.6$ M, which equates to about 3.1\% of Berkeley Lab's FY2004 projected operating and capital equipment budgets. This funding level was provided to develop new scientific ideas and opportunities and allow the Berkeley Lab Director an opportunity to initiate new directions. Budget constraints limited available resources, however, so a little less than $\$ 11.4 \mathrm{M}$ was expended for operating and $\$ 0.7 \mathrm{M}$ for capital equipment (2.5\% of actual Berkeley Lab FY2004 costs).

In FY2004, scientists submitted 175 proposals, requesting over $\$ 33.0 \mathrm{M}$ in total funding. Eighty-six projects were funded, with awards ranging from $\$ 16 \mathrm{~K}$ to $\$ 786 \mathrm{~K}$. These projects are summarized in the Table of Contents. 


\title{
Accelerator and Fusion Research Division
}

LB03009

\author{
Short Period Superconducting Undulator Development \\ Principal Investigators: Daniel R. Dietderich and Soren O. Prestemon
}

Project Description

Superconducting undulators (SCU's) have the potential to enable a new generation of insertion devices with significantly enhanced brightness and broadened energy range, representing important improvements over existing radiation sources. The most promising (though aggressive) technology is based on $\mathrm{Nb}_{3} \mathrm{Sn}$ superconductors.

An overarching goal of this project is to investigate performance characteristics and technological issues associated with the implementation of SCU's. Preliminary analysis considered both $\mathrm{NbTi}$ and $\mathrm{Nb}_{3} \mathrm{Sn}$ superconductors. Due to geometric constraints, high coil-pack current densities significantly enhance performance of SCU's. Our effort therefore concentrates on the use of high-performance $\mathrm{Nb}_{3} \mathrm{Sn}$ superconductors, which have the potential to provide the best performance. State of the art $\mathrm{Nb}_{3} \mathrm{Sn}$ conductors are a by-product of active research within LBNL's high-field dipole program.

The decision to focus on $\mathrm{Nb}_{3} \mathrm{Sn}$ superconductor for undulator designs was reinforced after collaborative discussions with fellow researchers from other light sources determined that image current heating may severely limit the performance of SCU's. The relatively high critical temperature $\left(\mathrm{T}_{\mathrm{c}}\right)$ of $\mathrm{Nb}_{3} \mathrm{Sn}$ serves to mitigate the risks associated with uncertainties in the magnitude of the image current heating and in the performance of the magnets cryogenic system.

\section{Accomplishments}

This project has resulted in a prototype device. It is a $14.5 \mathrm{~mm}$ period device and included a number of design modifications/improvements based on experience existing devices. The winding reversal method was modified to allow for constant tension on the conductor, significantly simplifying the winding process and reducing wear on the conductor insulation. Ceramic shims were developed at LBNL to improve cable stacking at crossovers and act as spacers in end coilpacks for precise positioning of the conductor. A ceramic insulation method, under development via small-business initiative, has been investigated and samples tested at LBNL. The insulation was not used on the prototype, but has the potential to significantly enhance the conductor fill fraction and hence the attainable performance. A key feature of the device was the implementation of trim coils to provide a field perturbation for phase error correction on future devices. Tests successfully demonstrated that the trim coils provide sufficient field variation to allow for phase error correction at all field levels. 


\section{Analysis and Modeling of Multicore Induction Cell Voltage Distribution Principal Investigator: William Fawley}

\section{Project Description}

The voltage distribution amongst the individual cores in a multi-core induction accelerator cell is not well understood by either physicists, electrical engineers, or (perhaps most importantly) accelerator builders. We propose to use both analytic and numerical simulation tools tools to investigate this problem, with specific attention paid to parameters/geometry relevant to the DARHT-2 accelerator cells. A solid understanding provides a good basis for choosing core/electrode dimensions for adequate safety margins to prevent electrical breakdown phenomena.

Our initial proposal suggested two means of attack for developing a greater understanding of the voltage distribution: (1) A simple analytic model in which one separates the problem into (coupled) inductive and electrostatic solutions. The induction voltages serves as a "source" for the electrostatic voltage and the capacitive loading from core-to-core and core to electrode surfaces affects the geometric distribution of the electrostatic voltage in the individual cores. (2) Numerical modeling involving the adaptation of of an existing electrostatic code and also the acquisition and use of a existing, fully time-dependent EM code ("LSP") written/supported by Mission Research Corp. both to validate the above analytic modeling and to compare predictions with experimental measurements of core-to-core voltage distribution being made in parallel at LBNL on a multicore test stand.

Accomplishments

Probably the most important accomplishment of this LDRD has been the detailed development of the analytic model. In collaboration with R. Briggs of SAIC, our model ties the microscopic properties of the core material (i.e. highly conducting, planar layers of magnetic material insulated by high dielectric material) to a coupled, macroscopic inductive and electrostatic solution. The results of the latter show that the capacitive coupling of the core to the outer conducting boundary of the accelerator cell can drive a "homogenous" term which in most circumstances decays exponentially with decreasing radius. The inductive drive powers an inhomogenous voltage component which, away from the outer radial boundary, tends to lead to an equipartition of longitudinal electric field gradient in the gaps between the individual cores. The mathematical solution depends upon a Green's function method with the number of independent eigenvectors and eigenvalues being equal to the number of inductive cores. Numerical evaluation of the analytical models showed good agreement both with experimental measurements made on the LBNL multicore test stand and also with results from numerical electrostatic and electromagnetic codes obtained at LANL and MRC for the DARHT-2 accelerator cell configuration.

We also obtained the LSP EM code from MRC and brought it up on a single user PC. Although we were able to run some simple test cases, the relatively long running times necessary for obtaining useful results for the DARHT-2 geometry persuaded us to concentrate on the analytic results and also the experimental measurements and analysis of same from the test stand. If need arises in the future, it would be possible to port the code to a multi-CPU LINUX cluster. We also wrote number of technical reports concerning our results. 


\section{Novel coherent THz and IR source using a laser wakefield accelerator and applications. \\ Principal Investigator(s): Wim Leemans}

\section{Project Description}

The purpose of this project is to investigate the production of coherent radiation in the Terahertz regime of the electromagnetic spectrum via the use of laser accelerated electron bunches. Experiments aided by theoretical modeling will aim at demonstrating that coherent $\mathrm{THz}$ radiation pulses can be produced with energy $0.01-0.1 \mathrm{~mJ}$ per pulse. Coherent $\mathrm{THz}$ pulses with peak source strengths several orders of magnitude beyond current technology are expected and will allow applications in new nonlinear regimes relevant to semiconductor and superconductor research.

Ultra-short $(<30 \mu \mathrm{m})$ electron bunches with high charge $\left(>10^{10}\right.$ particles/bunch) are routinely produced by laser-plasma acceleration at the l'OASIS laboratory. The electrons are generated in the self-modulated laser wakefield regime using a $10 \mathrm{~Hz}$, multi-TW Ti-Sapphire laser system by tightly focusing ultra-short (r 50 fs) laser pulses onto a high density $\left(>10^{19} \mathrm{~cm}^{-3}\right)$ gas jet. Since the electron bunch length is short compared to $\mathrm{mm}$ and sub-mm wavelengths, these bunches can be used to generate ultrashort pulses of coherent $\mathrm{THz}$ and far-IR radiation with an expected coherent enhancement of $>10^{10}$. In the first two years of this proposal we have generated $\mathrm{THz}$ radiation by relying on the generation of transition radiation at the plasma-vacuum boundary of the accelerator. Modeling indicates that within a $200 \mathrm{mrad}$ collection angle of the radiation and using a plasma with transverse scale length on the order of $0.5 \mathrm{~mm}$, in excess of 0.1 $\mathrm{mJ}$ will be produced, which is two orders of magnitude beyond current sources. The proposed source also has broad bandwidth and is absolutely synchronized with a TW CPA laser. The source will be utilized for investigation of atomic media (which includes gaseous and condensed media) with $\mathrm{THz}$ radiation.

\section{Accomplishments}

Our most significant accomplishment during FY04 was to measure the spectrum of radiation produced by transition radiation of laser accelerated electron bunches when they exit the plasma into the vacuum. The spectrum was obtained by using a scanning Michelson interferometer. The data were obtained by accumulating on the order of 5000 shots. By inverse Fourier transforming the autocorrelation function obtained with the interferometer a spectrum was obtained for experiments that used a single laser beam to generate the electron beam. Such electron beams have broad energy spread but contain large amounts of charge. The spectrum was found to peak near 1-2 THz. By fitting the data using the theoretical model developed in the first two years of the proposal, an electron bunch duration on the order of 50 fs was found.

We have also implemented a single shot technique based on electro-optic sampling. Here the THz radiation is first collected using an off-axis parabola (OAP), then demagnified using a matched pair of OAP's and mixed with a polarized short laser pulse in a non-linear birefringent ZnTe crystal. The amount of induced birefringence depends on the $\mathrm{THz}$ intensity. Monitoring changes in intensity of the polarized beam as it propagates through the crystal at the same time as the $\mathrm{THz}$ radiation allows determination of the field strength of the THz. 
Development of a DNA/RNA sequencing tool

Principal Investigator(s): Ka-Ngo Leung and Paul Richardson

\section{Project Description}

The goal of this project is to develop a plasma-nanopore system that can provide fast and accurate DNA or RNA sequencing. Since a nanopore can detect single molecules, a major advantage of the proposed method is that there is no need to amplify the target molecules. The DNA/RNA sequencing device is composed of a remote plasma source, a polynucleotide/plasma confinement region, a filtering plate containing nanopores, and a collection region for measuring the throughput of the filtering plate. The inert gas (helium or argon) plasma is generated by an $\mathrm{RF}$ source and is transported through a pipe to a confinement region. Helium or argon plasma is used because the negative ions of these inert gases are difficult to form. DNA (or RNA) strands, which have been prepared and suspended in a gaseous atmosphere, are introduced into the confinement vessel through a separate inlet. Thus, intermingling in this area are the organic molecules and the inert, cold plasma. A magnetic filter is placed in front of the extraction port in order to reduce the electron temperature. The cold electrons can attach to the polynucleotide strands to enhance their negative charge. A nanopore is bored through a conducting plate. Applying a voltage across this plate will draw the inherently negatively-charged strands as well as the electrons towards and through the pore. As in the aqueous case, as a strand traverses the nanopore, it should block the stream of electrons that can flow through the hole. These electrons are collected at a plate further downstream that is biased more positive than the filtering plate or plasma electrode. Measuring the electron current will reveal the blockage condition of the hole and, upon reliance of the physical uniqueness of the bases, can identify the sequential order of the bases.

\section{Accomplishments}

The plasma source and confinement vessel have been constructed and the plasma diffusion from the source to the confinement chamber has been studied. Plasma density and electron temperature have been measured using Langmuir- probes in order to optimize the plasma density and to achieve low electron temperature in the confinement chamber. With follow-on funding ways to insert DNA strands into the plasma and to manufacture nanopores will be studied.

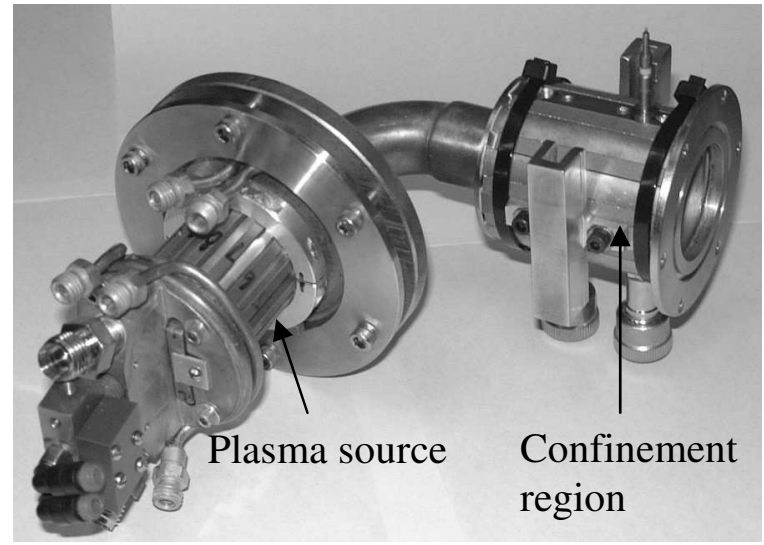

Figure 1. Constructed plasma source and confinement region
Electron Temperature in confinement chamber

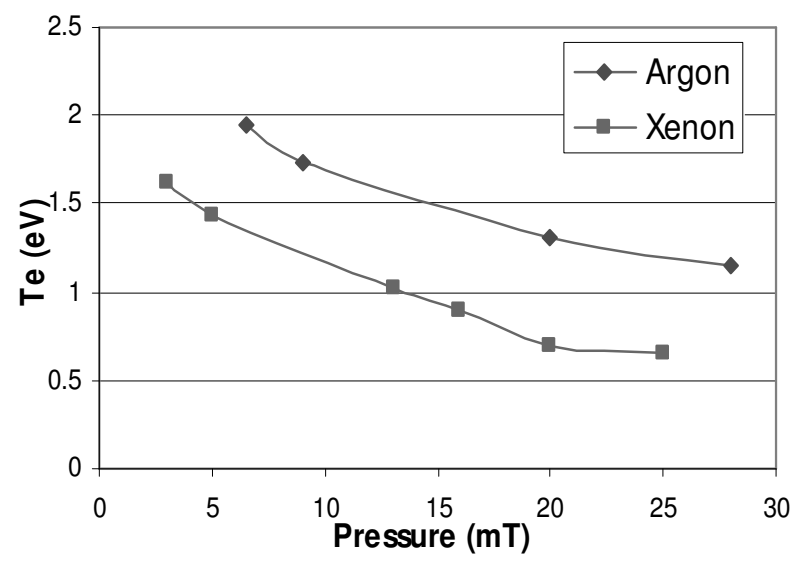

Figure 2. Measured electron temperature in the confinement region vs. source pressure 
Critical Accelerator Technologies for Advanced Light Sources

Principal Investigator(s): S. Lidia, A. Zholents, J. Corlett, S. Leone, H. Padmore, R. Schoenlein, R. Wells

Project Description

This project combines analytical, computational, and experimental approaches to develop concepts and technologies related to the production of x-rays in future accelerator-based facilities. We address the critical areas of soft x-ray production by cascaded harmonic generation, ultra-stable synchronization and timing techniques, high-power and high-brightness electron beam sources, and emittance control in high-brightness electron beams. A major component is the development of ultra-short pulse, soft $\mathrm{x}$-ray production by cascaded harmonic generation, enhanced self-amplified spontaneous emission (ESASE), and other free electron laser techniques. These schemes provide enhanced performance and capabilities over existing methods for EUV and soft x-ray production.

\section{Accomplishments}

We developed analytic and computational models of the harmonic generation FEL process that includes the two distinct stages: a modulating undulator driven by a laser seed and a radiating undulator driven by the pre-bunched electron beam. With these tools, we designed a four-stage harmonic cascade producing intense, coherent radiation with photon energies up to approximately $1.2 \mathrm{keV}$. The GENESIS code was used to model the performance of independent harmonic cascades driven by electron beams with energies of $1.1,2.1$ and $3.1 \mathrm{GeV}$. A set of design parameters has been developed with good performance over a range of photon energies from $40 \mathrm{eV}$ to $1.2 \mathrm{keV}$, with acceptable sensitivity tolerances.

We conceived and developed ESASE techniques to generate extremely high peak current ( 1-10 kA) micro-bunches within an electron beam bunch, with only limited beam quality degradation due to collective effects. For the LCLS undulator with a modified beta function $(12 \mathrm{~m})$, the much shorter gain length results in more rapid saturation of the radiation field, easing requirements on the LCLS electron beam quality. GINGER simulations show that single subfemtosecond $\mathrm{x}$-ray pulses are possible with a few-cycle carrier-phase-stabilized optical laser seed, providing ultra-short pulse capabilities with automatic synchronization to the seed laser.

Addressing a very significant issue in short pulse x-ray light sources, we designed and built a fiber optic synchronization link as a proof of concept for timing distribution. Piezo-based phase modulation techniques compensate for fiber length variations, preventing long-term drifts caused by thermal effects. Timing errors in the $100 \mathrm{~m}$ fiber were reduced from several picoseconds to 200 femtoseconds over several hours. We propose a set of novel techniques to reduce these timing errors to tens of femtoseconds and lower.

We have developed a drive laser concept to produce tailored, high-brightness electron bunches with the combined advantages of high stability, and precise control over the spatial and temporal intensity profiles of electron. Engineering design studies have demonstrated successful thermal management during high average power operation of an rf photoinjector cavity, enabling electron beam production up to $10 \mathrm{kHz}$ repetition rates. Theoretical and experimental studies of metallic $(\mathrm{Cu})$ and alkali semiconductor $\left(\mathrm{Cs}_{2} \mathrm{Te}\right)$ photocathodes, we have measured quantum efficiency and momentum spectrum of the photoemitted electrons, providing valuable input to our modeling and simulation codes. 
Optimal Solvers for Infinite-Dimensional Hamiltonian Systems

Principal Investigators: Esmond Ng and Robert D. Ryne

\section{Project Description}

We will develop optimal discrete models for infinite-dimensional Hamiltonian systems. We will use symplectic split-operator methods in the time domain, finite element-based and wavelet-based spatial decompositions, and we will examine both Hamiltonian and Lagrangian discretizations.

This work is motivated in part by the great success of symplectic integrators for classical finite-dimensional Hamiltonian systems. These have become indispensable for studying long-term behavior including the dynamics of charged particles in plasmas, the dynamics of stars in gravitational systems, and the dynamics of atoms and molecules in materials. Under this project we will develop new methods that can be applied to infinitedimensional systems governed by equations such as Maxwell's equations, the Schrodinger equation, and the nonlinear Schrodinger equation.

\section{Accomplishments}

Since the publication of the last year's report, we focused our efforts on the spatial discretization side of the project. Substantial progress has been made in developing and applying both wavelet- and finite element-based discretization schemes.

The principal accomplishment for the project in FY04 has been the successful design and implementation of a fully three-dimensional wavelet-based Poisson solver. An elliptic partial differential equation (PDE), the Poisson equation allows for testing discretization schemes in a setting with no time dependence (but possibly very complicated boundary conditions). The focus of our current work in this direction is optimization of algorithms in a way that will allow for simultaneous de-noising of the data sets and reduction in the computing time and storage requirements. The results obtained and insights developed in this setting are immediately applicable to the design of solvers for the hyperbolic PDE's representative of the infinite-dimensional Hamiltonian systems under study. In addition, the algorithms developed so far are being extended so as to allow for modeling synchrotron radiation and its effects on the dynamics of beams in accelerators and light sources.

Within the framework of finite element based discretization, we used a basis of continuously differentiable basis functions to implement a prototype one-dimensional symplectic solver. When tested on problems with analytically known solutions, this solver exhibited excellent energy conservation and pointwise accuracy properties over integration times as long as hundreds of thousands of the system's natural oscillation periods. This work is currently being extended to higher spatial dimentions. 


\section{Superconducting Magnet Systems for Ex-Situ NMR Spectroscopy \\ Principal Investigator: GianLuca Sabbi}

\section{Project Description:}

Superconducting magnets play a key role in nuclear magnetic resonance, a powerful spectroscopy (NMR) and imaging (MRI) technique with applications over a wide range of disciplines. In traditional NMR, superconducting devices have demonstrated the capability to provide significantly higher fields and better field quality than their resistive or permanent magnet counterparts. As a result, superconducting magnets hold the largest share of the multibillion dollar industrial market for NMR/MRI systems. The same performance advantages can be expected in advanced applications such as ex-situ NMR. In ex-situ NMR, a magnetic field is generated in an open space to acquire magnetic resonance information on samples which cannot be inserted in the bore of a magnet. The new configuration poses specific challenges requiring a new class of superconducting devices. In the first year of this LDRD project (FY03) the main design issues were addressed and a proof-of-principle prototype was fabricated and tested. The second phase (FY04) involved the design, fabrication and test of a prototype with high field quality, suitable to perform ex-situ NMR experiments. High resolution NMR techniques have applications to chemistry, physics, materials, industrial sensing, earth sciences, energy, environment, drug discovery. The development of ex-situ NMR will transform those applications and industries for which placing samples in a fixed static field is a severe impediment.

\section{Accomplishments:}

The reasearch program achieved the objectives stated in the proposal. The key design issue was to produce high field intensity and homogeneity in a volume located ouside the magnet structure. The design challenges involved: superconducting coil configuration and magnetic field optimization; magnetic force management and minimization of the coil support structure on the NMR analysis side; precision of the implementation, simplicity of fabrication, cost reduction and scalability from smaller to larger devices; minimization of the fringe field away from the volume of interest. Based on the results from last year, the design concept was significantly improved, and the magnet geometry was optimized for higher field and better field quality. The new design attains a magnetic field of 3400 Gauss with field homogeneity within $100 \mathrm{ppm}$, in a cylinder with $10 \mathrm{~mm}$ length and $2 \mathrm{~mm}$ radius, located at a distance of $45 \mathrm{~mm}$ from the superconducting coils. The design approach allows to tune the distance between the analysis volume and the coil, and to increase the length of the analysis volume by simply extending the length of the coils. After completing the design, a prototype was fabricated and tested. The magnet achieved $98 \%$ of its conductor-limited quench current after 15 training quenches. Magnetic field measurements performed in a room-temperature volume facing the magnet confirmed the design calculations. The next step (beyond the scope of this LDRD) will be to use the prototype superconducting magnet for high-resolution, ex-situ NMR experiments. Preparations for a first test have reached an advanced stage. 
Solid state quantum computer development with single ion implantation

Thomas Schenkel, and Jeffrey Bokor

\section{Project Description}

The goal of this work is to form qubit arrays by single ion implantation, to characterize the electrical properties of the implant structures, and to integrate dopant arrays with gate and readout structures through electron beam lithography. The key to demonstrating the possibility of a scalable solid state quantum computer is to show that it's basic building blocks can function. There is currently intense interest around the world to show for the first time that single spins in a solid can be controlled and read out. The idea is to gate control the spin polarization of the ${ }^{31} \mathrm{P}$ adjacent to the metal island in the SET. The source drain current in the SET can be tuned with the gate voltage and is very sensitive of the potential close to the metal island. This sensitivity allows the use of SETs as electrometers. Now the spin of the electron in the second ${ }^{31} \mathrm{P}$ atom can be prepared (gate controlled through Stark shift of the resonance in an external magnetic filed) to be parallel or anti-parallel to the detector ${ }^{31} \mathrm{P}$ spin. The gate between ${ }^{31} \mathrm{P}$ atoms is now used to pull the electron from the target qubit to the detector ${ }^{31} \mathrm{P}$ atom. This transfer is allowed only if the spins are anti-parallel, and this spin depended charge measurement allows the readout of the target spin state. This scheme was first propose by Kane in 1998; many materials and processing issues have not been resolved and several groups around the world are working on the first demonstration of it's practicality. We believe that our unique nano-processing capabilities will allow us to succeed in entering the regime of qubit testing.

\section{Accomplishments}

In the third and final year of our project we succeeded in integrating ion implantation with scanning probe alignment. Ion implantation with scanning probe alignment allows us to introduce dopants into nanometer scale device structures with high alignment accuracy and without parasitic implantation due to imaging with the ion beam. In this new instrument, we transport an ion beam through a small hole that is placed in the tip of the scanning probe. We developed a technique for formation of holes with diameters as small as $5 \mathrm{~nm}$ and have characterized ion transmission properties of nanometer scale holes. Detection of single ions was achieved for high charge state phosphorous, tellurium and bismuth ions with over $80 \%$ efficiency, and resists for single ion registration were tested.

In parallel, we demonstrated Coulomb blockage and gate modulation of source drain currents in silicon based single electron transistors (SET) in silicon-on-insulator. Dopant segregation effects in the presence of several $\mathrm{SiO}_{2} / \mathrm{Si}$ interfaces were identified and characterized.

The figure shows the LBL logo formed by ion implantation with scanning probe alignment. This new technique now allows us to form single dopant atom arrays in device structures for tests of quantum information processing schemes with quantum bits based on donor electrons or nuclei, or excited states single dopant / defect centers.

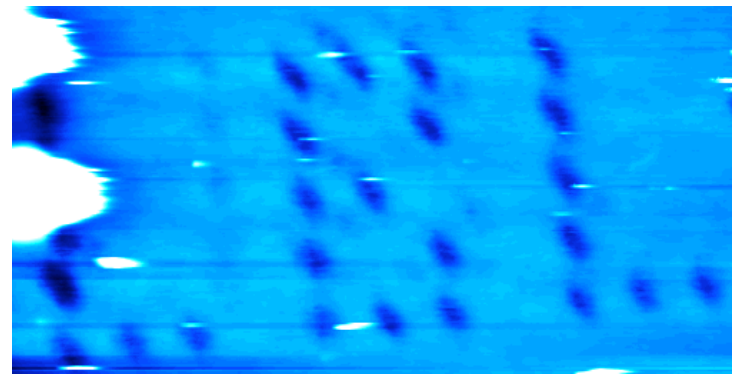

Dot patterns formed on silicon by ion implantation with scanning probe alignment ( $7 \mathrm{keV} \mathrm{Ar}^{2+}$ ions). Dot size is $400 \mathrm{~nm}$, limited by the hole in the scanning probe tip. Holes with diameters of $5 \mathrm{~nm}$ have been formed in tips. 
Electron Production and Collective Field Generation in Intense Particle Beams Principal Investigators: Miguel Furman, Jean-Luc Vay

(this is a joint LBNL-LLNL-VNL LDRD)

Project Description

Electron cloud effects (ECEs) are increasingly recognized as important, but incompletely understood, dynamical phenomena that can severely limit the performance of present electron colliders; the next generation of high-intensity rings, such as PEP-II upgrade, LHC, the SNS, and the SIS 100/200; or future high-intensity heavy ion accelerators such as envisioned in Heavy Ion Inertial Fusion (HIF). Deleterious effects include ion-electron instabilities, emittance growth, particle loss, increase in vacuum pressure, excessive heat load at the vacuum chamber walls, and interference with certain beam diagnostics. Extrapolation of present experience to significantly higher beam intensities is uncertain given the present level of understanding.

The joint LDRD project is laying essential groundwork for launching a comprehensive R\&D program including experiments, theory and simulations to better understand the phenomena, establish the essential parameters, and develop mitigating mechanisms. We are developing insights into the essential processes and models of the relevant physics, and implementing these models in computational production tools that can be used for self-consistent study of the effect on ion beams. We will validate the models and tools through comparison with experimental data, including data from new diagnostics to be developed as part of this work and validated on the High-Current Experiment (HCX) at LBNL and the STS500 test stand at LLNL. We will apply these models and diagnostic techniques to High-Energy Physics (HEP) and other advanced accelerators.

\section{Accomplishments}

We have begun to use the simulation tools developed in FY03-04 to provide a 3D description of the electron cloud and the resultant external field modifications and have applied it to gain a deeper understanding of electron cloud effects in specific accelerator modules, such as quadrupoles, gaps with and without acceleration, and sources. We have developed a promising technique for averaging over fast electron timescales, have implemented it in WARP, and have begun testing it in the full WARP environment. This technique may have broad application to other fields, including magnetic fusion and space plasma physics. The secondary emission routines extracted from POSINST and provided to Tech-X in FY03 have been released in a selfcontained package, named CMEE, available through Tech-X web site under a license for free non-commercial use.

Extensive studies of the effects of specified electron cloud distributions on the dynamics of a coasting ion beam in a 200-quadrupole system have led to the following conclusions: (1) Heavyion beams are surprisingly robust to electron clouds (a beam neutralization factor of at least several percent is needed for substantial beam degradation within 200 quads), (2) electron cloud density modulation, resonant with the beam-breathing mode, is the most dangerous perturbation, (3) elliptical distortions, resonant with beam quadrupole modes, increase beam emittance, (4) electron cloud effects on ion beam propagation is a rich, complex subject, and is not well characterized by a few beam moments

We have recently started the first self-consistent simulations of the magnetic section of HCX using the newly developed tools, including the large time-step electron mover, and have obtained encouraging qualitative agreement with experimental results. 


\section{Advanced Light Source Division}

LB04013

Coherent X-ray Diffraction Imaging (CXDI): progress report for the period Oct 12003 to Sept 302004

Malcolm Howells (LBNL, ALS), Henry Chapman (LLNL), Chris Jacobsen (SUNY), Janos Kirz (SUNY), Howard Padmore (LBNL, ALS), John Spence (ASU), Uwe Weierstall (ASU)

\section{Project description}

The goal of this work was to explore the limits of the applicability of a new high-resolution three-dimensional x-ray imaging technique, Coherent X-ray Diffraction Imaging (CXDI) to problems in materials and life sciences. For the history of the new technique see [Spence 2003] . In CXDI, a diffraction pattern is measured using coherent $\mathrm{x}$-ray illumination, and inverted to form a real-space image. Since a diffraction pattern has no direct information on phase, the inversion calculation must include use of a "phase-retrieval" algorithm, a procedure which has already been developed to an advanced level in visible-light optics. The advantage over imaging with lenses is that it eliminates limitations due to the efficiency, contrast-transfer and depth-offield properties of zone-plate $\mathrm{x}$-ray lenses. Like most ultramicroscopy techniques CXDI is limited by radiation damage for biological samples and by exposure time considerations for radiation-hard samples. The task in this project was to critically evaluate these issues, and show feasibility of CXDI for future ALS programs.

The experimental goal was thus to make the above assessments for representative specimens including both biological objects such as whole cells and man-made nanoscale structured materials, such as those of interest for catalysis and hydrogen storage. For each of these systems we needed to demonstrate high-resolution CXDI and determine the required exposure time as a function of the resolution.

\section{Progress report}

During the reporting period we have made significant progress on the above goals. Specifically we have

- Remodeled ALS beam line 9.0.1 with a new monochromator suited to CXDI

- Constructed a small experimental chamber for trial experiments on 9.0.1 and used it to record the first 2D diffraction patterns of test objects

- Made successful reconstruction of the object in 2D at $30 \mathrm{~nm}$ resolution using a new variant of the standard phase-retrieval algorithm (known as "shrinkwrap" [Marchesini 2003]).

- Engaged in a collaboration with the Stony Brook x-ray microscopy group who had developed a diffraction chamber suitable for life-science CXDI. Installed this chamber at ALS (which is the best soft $\mathrm{x}$-ray source for CXDI)

- Begun a program using yeast cells as representative biological samples. First 2D results: 30 $\mathrm{nm}$ resolution images achieved on freeze-dried yeast with a dose of $10^{8}$ Gray. Note theory indicates 3D dose will be the same as 2D for the same resolution and statistical accuracy.

- Yeast results extended to eight views (quasi 3D (viewable as stereo pair and as a movie on the internet [Thibault 2004])) and to frozen-hydrated samples

- Experiments on 3D gold test objects completed successfully with 130 views including true 3 D reconstruction (i. e. not serial 2D); viewable as a movie on the internet [Chapman 2004]

- First 3D measurements on a challenging materials-science sample, a $\mathrm{Ta}_{2} \mathrm{O}_{5}$ foam, essentially an aerogel of $1 \%$ density, 130 views in 20 hour exposure, reconstruction in progress

- Estimated the radiation-damage limit to life-science resolution as $10 \mathrm{~nm}$ [Howells 2004] 
A Novel Ultra-High Resolution $(<=10 \mathrm{meV})$ Inelastic Scattering Spectrograph to Study Coupled Electron-Phonon-Orbiton Interactions in Degenerate Quantum Systems

Principal Investigators: Zahid Hussain, Eric Gullikson and M. Zahid Hasan

Project Description:

The purpose of this project is to design, fabricate and commission a prototype ultra-high energy resolution inelastic (energy resolved) soft X-ray scattering (photon-in/photon-out) spectrograph near the $\mathrm{M}$ edge of 3d transition metal oxides. The scientific scope is to utilize such novel high energy resolution to study intricate interplay between various low energy excitations, such as orbiton (orbital fluctuation), phonon, polaron and magnons, in highly correlated electron systems such as quantum degenerate colossal magnetoresistive (CMR) oxides and high temperature superconducting cuprates. Understanding the competition-cooperation behavior among these excitations is believed to be the key to CMR effect and other phenomenon in $3 \mathrm{~d}$ transition metal oxides.

The optical design of the spectrograph consists of three major parts: a spherical focusing mirror, an aberration correction variable line spacing (VLS) grating and a high quantum efficiency in-vacuum CCD detector. To maximize overall throughput, slit-less design is adopted, which requires a 4 micron well focused synchrotron beam on the target. The overall length of spectrograph is $2 \mathrm{~m}$ and the operative photon energy range is between 30 to $120 \mathrm{eV}$. This spectrograph will be used as a main part of the experimental endstation in conjunction with the ultra-high resolution beamline (MERLIN at sector 4.0).

Accomplishments:

Optical design of the spectrograph was done to demonstrate the feasibility of achieving resolving power 10,000 at $\mathrm{Mn} \mathrm{M}$ edge (47eV, see LDRD 03 report). The corresponding 20001/mm VLS grating was ordered, received and measured at beamline 6.3 .2 by Eric Gullikson. The efficiency curve shown in figure 1 (left panel) agrees with theoretical prediction very well. Such high efficiency grating is essential for photon-hunger spectrograph. The mechanical design was done and the internal parts of monochromator chamber were completely fabricated (right panel in figure 1). The encoder system for grating drive mechanism and the bench-top angular calibration were tuned to ensure perfect initial setup. Proposal for follow-on funding for the initial performance test has been submitted and beamtime has been granted at BL12.0.1 for next cycle (January 2005-June 2005).
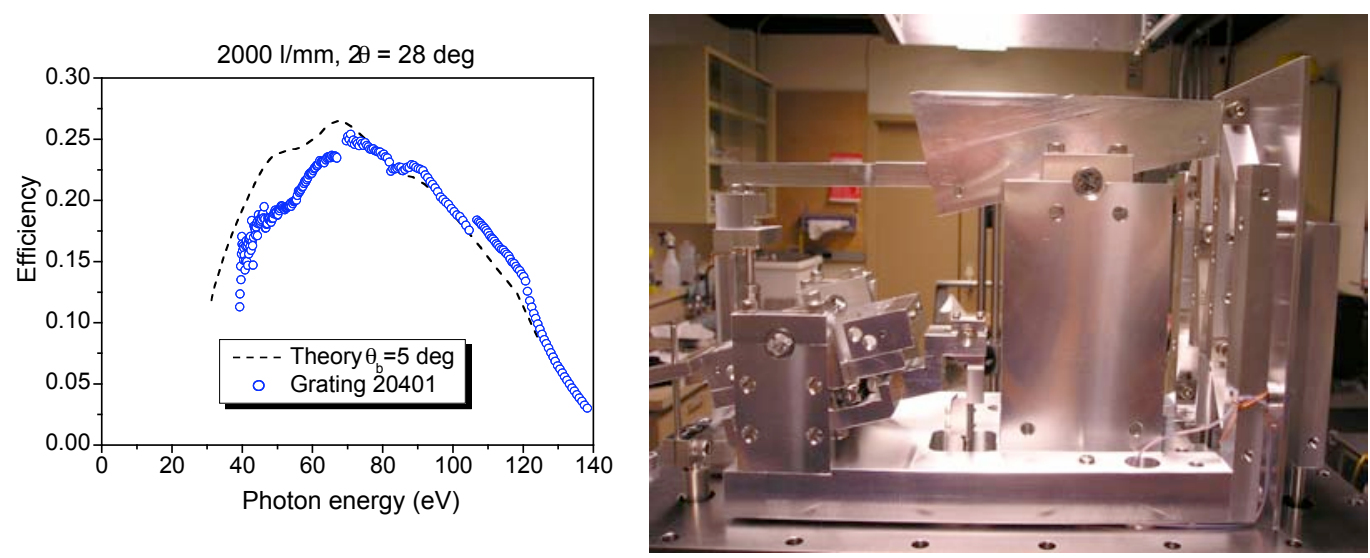

Figure 1: Left: Grating efficiency measured at BL6.3.2. Right: interior of spectrograph Right: interior of optics chamber. 


\section{Lensless Imaging of Yeast Cells \\ Principal Investigator(s): Janos Kirz}

\section{Project Description}

Our goal is to develop the technique of Diffraction Microscopy, and image frozen hydrated yeast cells (Saccharomyces cerevisiae) in three dimensions at about $20 \mathrm{~nm}$ resolution. The improvement in resolution compared to what has been accomplished (or is likely to be accomplished in the foreseeable future) by other techniques is expected to provide insight into the life cycle and the interrelation of the organelles within this important model organism. We use the apparatus built at Stony Brook and Brookhaven and installed on BL 9.0.1 at the ALS during the fall of 2003.

\section{Accomplishments}

During the period of this LDRD, with collaborators at Stony Brook and Cornell, we reconstructed diffraction patterns from freeze-dried specimens. The reconstructions, from 9 angular orientations of the specimen provide limited depth information, and establish the reliability of the method. We also collected diffraction data from frozen hydrated yeast cells, to establish the experimental parameters required for successful reconstruction. To this end we installed a second zone plate monochromator that operates in the "water window", at $530 \mathrm{eV}$, and developed the techniques necessary to control the thickness of the ice layer in preparing the specimen. In addition we studied the damage threshold as a function of resolution both in freeze-dried and in frozen hydrated yeast cells. The former show significant shrinkage, similar to that observed in electron microscopy. The latter are observed not to shrink. 
Development of a Coherent Far-IR Synchrotron Source at the ALS Principle Investigator(s): Michael C. Martin, John Byrd, Fernando Sannibale, Wayne R. McKinney, and David Robin

\section{Project Description}

The purpose of this project is twofold: (1) to further our understanding of the generation of coherent synchrotron radiation through experiments at the ALS using unique modes of operation of the ring, and (2) to consider a preconceptual design of a dedicated infrared storage ring to be located at the ALS. A source in the far IR that is much brighter than what is available at present will open up new possibilities for research in this traditionally difficult energy range. We intend to characterize super-radiant far-IR radiation from various novel modes of ring operations, including further experiments with femtosecond laser slicing. This research will be carried out with the goal of understanding how to create stable, coherent far-IR radiation from a storage ring. This will also lead to generation of a pre-conceptual design for a possible future infrared storage ring.

\section{Accomplishments}

The most significant experimental accomplishment of the year was the detailed studies of coherent synchrotron radiation being emitted from a femto-second laser sliced electron beam. This series of complex experiments built upon the unique laser slicing capabilities at the ALS where a femto-second laser is co-propagated with the electron beam through a wiggler. This gives this small "slice" of the electron beam a kick in energy which physically separates from the main bunch after traveling through dispersive sections of the accelerator (such as bend magnets). This leaves an electron bunch with a femto-second scale dip in its distribution, and this density modulation emits coherently in the far-IR. We were able to measure this far-IR radiation from two different ports at the ALS to demonstrate that significant intensity is observed, and the spectral profile changes as the electron bunch travels around the ring and this density is smoothed out. Theoretical calculations match well with our experimental results, and a paper will soon be submitted to Physical Review Letters.

We also continued detailed model development and conceptual design work for a dedicated coherent far-IR facility at the ALS: CIRCE (Coherent InfraRed CEnter). This included two publications on the stable bunch distortion that allows higher frequency coherent emission than the nominal bunch length would allow. This theoretical model fits well with experimental measurements we assisted with in 2003 at the BESSY synchrotron in Berlin, Germany. This detailed model, along with our understanding of a bursting instability threshold from our LDRD work in 2002, allowed us to present a fully refined accelerator design for CIRCE in two publications and at relevant conferences.

The scientific opportunities in the far-IR (terahertz) region were explored at a DOE/NSF/NIH workshop primarily stimulated by our presentation of CIRCE to a DOE BESAC subcommittee. This workshop was held in February 2004, and recently the report from the workshop was released detailing a large variety of scientific opportunities for new THz sources. CIRCE was given several pages of this report and was mentioned several additional times. This workshop and its report are key to our efforts to secure funding for CIRCE. 


\author{
Aberration Correction of Electron Microscopes \\ Principal Investigators: Howard Padmore, David Robin, Ulrich Dahmen
}

\title{
Project Description
}

We will focus our efforts on establishing the next-generation aberration corrected electron microscopy specifically photoemission electron microscopy (PEEM) and transmission electron microscopy (TEM). Aberration correction in PEEM can lead to resolution an order of magnitude better than today and a transmission two orders of magnitude better than today's systems at moderate spatial resolution. To surpass the 0.15 nanometer resolution of state-of-theart TEMs presently requires electron holography or focal series restoration together with complex image processing. Aberration correction in TEM provides the opportunity to go well beyond current limitations. Corrected electron optics for PEEM and TEM will enable significant advances in nanometer and atomic-scale characterization of materials.

The ultimate resolution of electron microscopes is limited by aberrations as well as by electron diffraction. The issues to be addressed are underlying assumptions of Scherzer relating to (1) static fields, (2) axially symmetric systems, (3) no space charge, and (4) no change of sign for the axial ray velocity.

Similar techniques can be applied in many areas of electron imaging; for example, we are starting a study of correction of the temporal spreading in streak cameras caused by chromatic nature of $\mathrm{x}$-ray photocathodes. By eliminating this temporal spread caused by differing flight times of electrons of different initial energy, the temporal resolution should improve from the picosecond to sub 100 femtosecond level and enable a new generation of ultra fast experiments.

Accomplishments

This work leveraged the presence of Prof. Harald Rose, the world expert on aberration correction for microscopes, and his former student Dr. Peter Schmid whose expertise has been in designing and analyzing schemes for aberration correction in electron microscopes and electron lithography devices.

In electron microscopes, the front-end optics is mostly responsible for generating the spherical and chromatic aberrations. To compensate for the aberrations requires that one or more of Scherzer's assumptions needs to be broken. In aberration corrected X-PEEMs, one technique is to bend the electron trajectories with a separator and then reflect the electrons in an electron mirror. This violates the axial symmetry and unidirectional assumptions of Scherzer. The addition of the corrector greatly increases the sensitivity to errors and the complexity of tuning the microscope. Differential algebra (DA) maps were developed and employed to study these assumptions.

The most critical component in such an aberration scheme for PEEM is the Separator magnet that bends the beam. The design of the Separator magnet for the present aberration corrected microscopes was one developed by Prof. Rose. The complexity of this component far exceeded the complexity of any of the other components of the microscope. DA mapping techniques were developed to analyze the sensitivity and correction algorithms for the beam Separator. Also a new concept of a separated function beam separator was developed that provides similar performance as the original Separator with significantly relaxed construction tolerances. This new Separator design has many advantages over the previous design. The new Separator simplifies the design, lowers the total cost, and increases tunablity during operation. 


\title{
Simulations of Femtosecond X-ray Spectra of Photoexcited Molecules
}

\author{
Principal Investigators: S. Mukamel ${ }^{\mathrm{a}}$, R.W. Schoenlein ${ }^{\mathrm{b}}$, T.E. Glover ${ }^{\mathrm{c}}$ \\ ${ }^{\text {a }}$ Chemistry Department, University of California, Irvine \\ ${ }^{\mathrm{b}}$ Lawrence Berkeley National Laboratory, Materials Sciences Division \\ ${ }^{\mathrm{c}}$ Lawrence Berkeley National Laboratory, Advanced Light Source
}

\section{Project Description}

The goal of this LDRD is to develop a theoretical framework for simulating and interpreting ultrafast $\mathrm{x}$-ray absorption spectra on the $100 \mathrm{fs}$ time scale. This theoretical work is driven by new experimental capability for generating ultrashort x-ray pulses from the ALS. Time-resolved spectroscopy with femtosecond $\mathrm{x}$-ray pulses will open new areas of research in physics, chemistry and biology by allowing the motion of atoms in materials to be directly probed on the time scale of a vibrational period which is the fundamental time scale for structural dynamics. At present, there is a strong need for theoretical support to interpret time-resolved $\mathrm{x}$ ray absorption spectra and to guide the experimental program at the ALS

Nonlinear spectroscopy in the visible regime is successfully described using the welldeveloped machinery of nonlinear response functions (NRF), which are expressed as combinations of multiple-time correlation functions of the dipole operator and provide the most compact and general formulation of optical signals. This proposal will focus on extending this approach to coherent nonlinear ultrafast x-ray spectroscopies with the goal of extracting information about chemical bonding and structure, the creation and breaking of bonds and other electronic and nuclear motions on the ultrafast time scale.

\section{Accomplishments}

This project is a vehicle to establish a collaboration between the theory group of Prof. Shaul Mukamel at U.C. Irvine, and the ultrafast $x$-ray spectroscopy group at LBNL. The LDRD funds a highly-qualified postdoc (DR. L. Campbell), with a background in x-ray absorption theory and modeling, who is synthesizing the machinery of nonlinear response functions and the corresponding computer codes that have been developed in the Mukamel group, with models for predicting x-ray absorption.

During the past year, Dr. Campbell has completed the development of a new computer code, XSNAP, for assisting in the simulation of x-ray absorption spectra of molecules in the excited state. XSNAP uses the quantum chemistry code GAUSSIAN to determine the excitedstate electron distribution. This distribution is then introduced to the $\mathrm{x}$-ray absorption code FEFF. The FEFF algorithm calculates the XAS in several independent steps using the excitedstate charge distribution and associate scattering potentials around the atoms determined from GAUSSIAN.

These codes have been applied to calculate the near-edge x-ray absorption spectrum of an important metal-to-ligand charge transfer molecule (Ru-bpy), following photoexcitation. The calculations show a shift of the primary absorption peak and the appearance of a new absorption feature due to the hole created by the optical excitation. The calculations are in excellent agreement with recent time-resolved x-ray absorption measurements of this molecule. This agreement confirms the validity and utility of the codes. In the future, we hope to apply these modeling techniques to understand $\mathrm{x}$-ray measurements of structural dynamics in other molecules and solids. 


\title{
Ultrafast Magnetization Dynamics
}

\author{
A. Scholl, H. A. Padmore, J. Stohr, T. Young, E. Glover
}

\section{Project Description}

The purpose of this work is to develop techniques for the study of ultrafast dynamics in magnetic materials. On its own this is of forefront scientific interest, but it also offers a new and very exciting area for early exploitation on the ALS f-sec slicing source as well as future initiatives. The goal is to demonstrate that we can measure spin and orbital moments in magnetic systems as a function of time after laser or magnetic field excitation on a p-sec streak camera, and to investigate the range of scientific applications possible for such a revolutionary new tool.

$\mathrm{X}$-ray pulses from the ALS are typically $80 \mathrm{psec}$ long, and so to achieve one p-sec time resolution, we will use streak camera detection. In order to measure and decode spin and orbital components of the magnetization, we will use the x-ray spectroscopic techniques of magnetic circular and linear dichroism. To achieve fast excitation, we require small excitation areas, and so we will use x-ray microfocusing techniques. Magnetic field excitation will use currents generated from laser driven GaAs current sources. A laser will also be used for direct electronic excitation. All of these techniques exist in some form at the ALS, and the approach here is to bring them together to attack this new area of x-ray spectroscopy applied to psec magnetization dynamics. The team of PIs on this LDRD has expertise in all of the relevant areas.

\section{Accomplishments}

All components needed to conduct first experiments were obtained and were installed at BL4 of the Advanced Light Source. A high-power f-sec laser system was set up. Significant time was spent on designing and optimizing the streak camera optics and on studying the constraints that currently limit the streak camera resolution. All components of the complex optics were tested. The streak camera was tested and promises to achieve the required temporal resolution. An experiment chamber, including a sample cryostat, an electromagnet, and diagnostics was designed and built. A laser enclosure was installed, which contains the laser beams and prevents accidental exposure to laser radiation.

First x-ray experiments were conducted in August 2004 during 2-bunch mode operation of the ALS. The timing of the pump laser, of the synchrotron pulse, and of several optical delay stages was optimized, which is a very important step towards a pump-probe experiment. F-sec UV pulses were used to check the function and the time resolution of the streak camera. Ni samples were produced and studied in an X-ray transmission geometry. X-ray absorption spectra of good quality were obtained from the streaked $\mathrm{x}$-ray pulse. This was a very important milestone, demonstrating the feasibility of ultrafast experiments using streak camera detection at the low repetition rate of the pump laser $(5 \mathrm{kHz})$. The necessarily low rate of the high power laser is responsible for a severe loss in x-ray intensity of about 5 orders of magnitude compared to static experiments, which could have prevented successful dynamics experiments. The results confirmed a sufficient x-ray signal. First IR pump - x-ray probe experiments were conducted and it was found that an improved electron detector would be needed. This new detector will be available for experiments in FY 2005. The results were presented at international conferences and at an ALS Scientific Advisory Committee meeting to make the case for an ultrafast magnetism center using the ALS slicing source. 


\title{
Developing Dynamic Soft X-ray Scattering to Probe Spatial and Temporal Fluctuations in Nanomagnets
}

\author{
N.V. Smith (ALS), S.D. Kevan, K. Chenel (ALS), J. Kortright (MSD)
}

\section{Project Description}

The aim of this project is to demonstrate the true potential of coherent soft x-ray scattering experiments to the study the dynamical properties of nano-objects, particularly in the area of nanomagnetism. We have delineated the optimum configuration of a beamline and dedicated end-station that will spawn an active and entirely new capability at the ALS. We have also further developed the scientific case for a new elliptically-polarizing-undulator (EPU) beamline dedicated to coherent soft X-ray imaging and scattering science in the ALS upgrade that has been proposed as part of the DOE Office of Science 20-year roadmap.

It is increasingly clear that complexity in a variety of material systems emerges on the mesoscopic length scale and can be manifested by anomalous dynamical phenomena and memory effects. We have therefore focused particularly on developing the soft x-ray analog of dynamic light scattering, so that the temporal dynamics of nanometer-scale spatial fluctuations in various complex materials can be explored. The experiments were conducted on a newly commissioned coherent soft x-ray side branch of beamline 12.0 at the ALS - the first beamline optimized for coherent soft x-ray science in the world.

\section{Accomplishments}

This year's research has resulted in several important accomplishments along the road to meeting our goals. The coherent magnetic scattering beamline and end station were commissioned and the first experiments started. The beamline delivers very nearly the theoretically-expected coherent flux, given the ALS emittance and the efficiency of the optics. The end station is able to produce a magnetic field to $0.6 \mathrm{~T}$ in arbitrary orientation, while keeping the sample position stable with micron precision. A key feature of the apparatus is the positional stability of our spatial filter pinhole relative to the scattering medium, and we have shown that this is adequate as well.

Part of a soft x-ray speckle pattern of a hexagonal array of cobalt nanoparticles collected at the $\mathrm{Co}_{3}$-edge resonance. The radius of the arc of light corresponds to the Bragg angle for diffraction off the imperfect and polycrystalline nanoparticle lattice having a lattice constant of $14 \mathrm{~nm}$. The grainy structure is speckle that arises from use of a transversely coherent $\mathrm{x}$-ray beam.

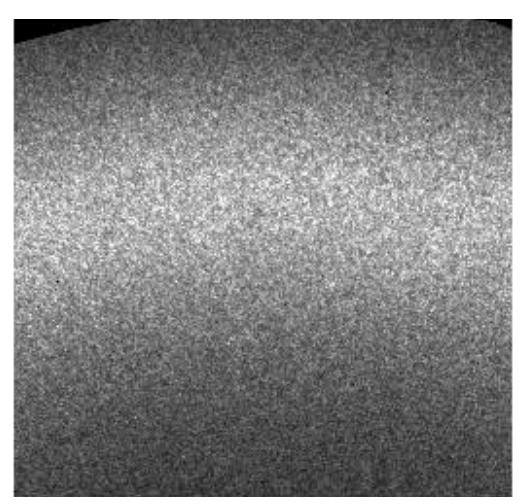

An important step has been to produce soft x-ray speckle patterns of high fidelity, as shown in the figure. This shows part of a speckle/diffraction pattern from a hexagonal array of cobalt nanoparticles at the L-edge wavelength of $1.6 \mathrm{~nm}$. We have made progress in understanding the charge and magnetic scattering contrast and in using the magnetic contrast to probe microscopic memory effects and magnetic fluctuations. 


\author{
High Average Power Laser Amplifier \\ Principal Investigators: Russell Wilcox and Robert Schoenlein
}

\title{
Project Description
}

The purpose of this project is to develop a titanium:sapphire laser amplifier capable of providing tens of Watts average power at tens of $\mathrm{kHz}$ repetition rate, with high efficiency and excellent beam quality. Typically, repetition rates for high power amplifiers are much lower, which tends to reduce the effect of thermal lensing because of the larger beam size required for efficient energy extraction. In the high pulse rate case, a smaller beam size results in stronger thermal lens effects, requiring low temperature cryogenic cooling and careful optical design. Development of this amplifier will provide a compact source of moderate energy pulses at high repetition rate, enabling high data rate femtosecond optical experiments, including applications in synchrotron femtosecond $\mathrm{x}$-ray pulse generation.

We will develop a regenerative pre-amplifier and a two-pass power amplifier based on cryogenically-cooled ti:sapphire. The amplifiers will be designed to generate $\sim 60 \mathrm{~W}$ of average power at a repetition rate of $40 \mathrm{kHz}$, although lower rates are possible. Special cooling chambers for the 2-pass amplifier and regenerative amplifier will be designed, utilizing compact cryorefrigerators. Measurements will be made of the output power and energy, thermal lens focal length, and influence of the cryorefrigerator mechanical vibrations on the beam.

\section{Accomplishments}

We have built an amplifier and tested it at a pump pulse rate of $10 \mathrm{kHz}$, pump power of $90 \mathrm{~W}$ and a signal rate of $1 \mathrm{kHz}$, producing $2 \mathrm{~mJ}$ of energy per pulse and a saturated gain of 5 in two passes. Measuring the cooling capacity of the cryorefrigerator and the absorption of the $10 \mathrm{kHz}$ pump, we have shown that pumping at $40 \mathrm{kHz}$ with four $90 \mathrm{~W}, 10 \mathrm{kHz}$ pumps will result in a crystal temperature of under $100 \mathrm{~K}$, sufficient to reduce thermal lensing to acceptable levels. We have modeled the thermal lens at various pump powers to confirm this, using finite element analysis of the thermal distribution in the crystal.

We have also developed a regenerative pre-amplifier operating at $10 \mathrm{kHz}$, and demonstrated $400 \square \mathrm{J}$ per pulse, sufficient to test the amplifier at its maximum output energy. The regenerative amplifier is capable of $40 \mathrm{kHz}$ operation, using a recently-developed high frequency Pockels cell switch.

An important accomplishment has been the demonstration of 2-pass amplifier operation with a high-capacity cryorefrigerator. These refrigerators produce large amplitude mechanical vibrations, which we have sucessfully isolated from the optical table on which the amplifier is mounted. Measurement of the optical table deflection when the cryorefrigerator is operating show only a few microns deflection of laser beams reflected from optics on the table. This solves problems which previously inhibited the use of such refrigerators in sensitive applications. 


\section{Chemical Sciences Division}

LB04038

Gas phase studies of the building blocks of life

Principal Investigator: Musahid Ahmed

\section{Project Description}

The purpose of this project is to study the fundamental properties of molecules that are the building blocks of life. Dipole moments, ionization energies, electron affinities, proton affinities, and electronic structure all contribute to the photochemistry, radiationless processes and reactivity necessary for biological functions. Both intramolecular properties (how a molecule interacts with itself) and intermolecular properties (how a molecule interacts with its surroundings) are unknown for a great majority of biomolecules. The dynamics and reactivity of peptides, proteins, and nucleic acids must also be probed to understand biology and medicine at the molecular level.

We will generate molecular and particle beams of biomolecules and perform time-of-flight mass spectrometry and photoelectron spectroscopy with tunable vacuum ultraviolet (VUV) light to measure these properties. We will demonstrate our capabilities of studying the structure and dynamics of these biomolecules using IR radiation with an eye towards controlling reactivity. The knowledge and skills that we will gain from this project will provide us with the tools to study other complex systems - aerosols, polymers, thin films, large clusters and heterogeneous processes on surfaces.

Accomplishments

Two methods have been developed successfully to transport fragile biomolecules to the interaction region of a time of flight mass spectrometer. In the first instance, intense focused particle beams, generated by aerosol methods and aerodynamic focusing, were impacted on a thermal heater leading to fragment free generation of amino acids. Arginine and Asparagine, amino acids which are notorious for fragmentation in mass spectrometers, were detected intact in their unprotonated form and their ionization energies determined for the first time. Ionization energies for Phenylalanine, Tryptophan, and Glycine in the gas phase were recorded. VUV photoionization of two di-peptides, Trp-Gly and Gly-Gly, were performed and novel fragmentation mechanisms were observed. These experiments are being extended to polypeptides with an eye to performing sequencing mass spectrometry.

The second method employed at the beamline is laser desorption used in conjunction with supersonic jet cooling in a molecular beam to generate biomolecules with very little internal energy in them. This method has been applied successfully to detect fragment free DNA bases (Guanine, Cytosine and Adenine). It is apparent that jet cooling is necessary, otherwise there is extensive fragmentation. Ionization energy onsets were measured as a function of cooling, providing for the first time mass spectrometry ionization energy determination for these molecules. The coupling of a high repetition rate IR laser to the desorption system will allow unprecedented access to the conformational landscapes of these large biomolecules in the future.

In both cases, the success of the project stems from coupling intense beams of molecules and particles to tunable VUV radiation, and future experiments will allow unprecedented access to chemical information. 
Disorder and Multiple Length Scales in Non-Fermi Liquid $f$-electron Intermetallics Principal Investigation: Corwin H. Booth

\section{Project Description}

The purpose of this project is to study the relationship between local lattice order and the socalled "non-Fermi liquid" (NFL) properties that occur in many $f$-electron intermetallic systems. These properties include such behavior as a logarithmically diverging linear coefficient of the heat capacity and the magnetic susceptibility. Although some NFL materials are known to be structurally well ordered, both theoretical and experimental evidence exists that disorder plays a role for a wide range of NFL compounds. Disorder affects the magnetic and electronic properties in these models by varying the degree of hybridization between the $f$-orbitals and the conduction electronic states $(V)$, essentially by creating a distribution of hybridization strengths. In addition, local variations can also occur in the density of states at the Fermi level. We explore this hybridization by applying x-ray absorption fine structure (XAFS) and pair-distribution function analysis of powder diffraction data to determine local distributions of bond lengths $\left(V \sim 1 / r^{n}, n\right.$ is a function of the orbital angular momenta), and applied pressure techniques to change $V$ and $r^{n}$ in a more controlled fashion. In addition, we measure materials that can perturb the local environments in particular ways. These include chemical substitution onto particular lattice sites and varying particle size.

\section{Accomplishments}

We have made three significant accomplishments in FY2004. The first was to continue our understanding of $\mathrm{CeAl}_{2}$ and $\mathrm{CePt}_{2+x}$ nanoparticles. We have determined that the large distortions we measured in FY2003 were most likely due to off-center displacements of the Ce within the Al or Pt cage, similarly to what occurs in many substituted buckyball systems. Such an off center distortion is likely precipitated by the surface in these $4-8 \mathrm{~nm}$ particles. Modeling the effect on $V$ indicates that such a distortion (off center displacement with no other distortions) can only increase $V$. Since the Kondo temperature is observed to decrease in the $\mathrm{CeAl}_{2}$ nanoparticles, this result indicates a concomitant decrease in the density of states at the Fermi level must also occur. Further work has been delayed because the $\mathrm{CePt}_{2+x}$ nanoparticles were found to have trace magnetic impurities present. These impurities should not affect the structural result, so measurements on new samples with allow the project to be completed.

Another major accomplishment was the measurement of the Sn substitution site in $\mathrm{CeCoIn}_{5-x} \mathrm{Sn}_{x}$. CeCoIn $n_{5}$ is a heavy-fermion, NFL superconductor. Substitution of Co with $\mathrm{Rh}$ or Ir affect $\mathrm{T}_{\mathrm{C}}$ slowly. For instance, CeIrIn $\mathrm{n}_{5}$ has only a slightly smaller $\mathrm{T}_{\mathrm{C}}$ and $\mathrm{CeRh}_{1-x} \mathrm{Co}_{x} \mathrm{In}_{5} \operatorname{loses}$ superconductivity only after about $85 \% \mathrm{Rh}$. Sn substitution, on the other hand, fails to superconduct after only $\sim 3.5 \% \mathrm{Sn}$ for Indium $(x=0.18)$. In a three-dimensional superconductor, impurity scattering would be similar for, say, Ir and Sn doping. However, our local structure studies show that Sn preferentially substitutes onto the Ce-In plane. Together with a wealth of circumstantial evidence for two-dimensional superconductivity in $\mathrm{CeCoIn}_{5}$, these results provide a route to directly perturb the superconducting state in the superconducting planes.

Finally, we obtained our first Ce K-edge XANES measurements in a diamond anvil cell on the HP-CAT at the Advanced Photon Source (Argonne National Laboratory). These measurements looked for a valence transition that was expected to occur when the system becomes superconducting above about $2 \mathrm{GPa}$. We observed no such transition up to $18 \mathrm{GPa}$. This experiment also opens the door to many similar measurements on mixed valence systems. 


\section{Coherent Control and Quantum Information in Polyatomic Molecules \\ Principal Investigator(s): Stephen R. Leone}

\section{Project Description}

The ability to track the fate of a molecule, in real time, during a chemical reaction has only been possible since the advent of ultrafast lasers. Studies in this field have not only provided dynamical information pertaining to the particular pathway a molecule follows during a chemical reaction and the timescales of these, but they also give detailed insight into the complex interplay between electronic, vibrational and rotational degrees of freedom in both diatomic and polyatomic systems.

The present chemical physics effort is focused on two key questions. Firstly, by exciting well-defined multimode vibrational wavepackets in the ground state of jet-cooled polyatomic molecules, can researchers guide the molecule along a particular reaction channel? And if so, what does this tell us about the nature of the chemical bond being broken? Secondly, by exciting well-defined vibrational wavepackets, are we able to control one motion over another (say a bend versus a stretch) using sequences of ultrafast laser pulses and tailored ultrafast laser pulses; can the work on quantum information be extended from atoms and diatomic molecules to more complex polyatomic molecules with multiple degrees of freedom?

\section{Accomplishments}

One significant accomplishment this year was to rotationally align diatomic molecule wavepackets in $\mathrm{O}_{2}$ and $\mathrm{H}_{2}$ under jet-cooled and room temperature conditions. The timedependent alignment exhibited by these molecules could be further enhanced by a timedelayed controlled laser pulse. The control pulse triggers further Rabi-cycling within the rotors and is only effective at time-delays corresponding to recurrences of the initial ensemble alignment. This illustrates how sequences of pulses can be effectively used to coherently modify the dynamics of a system. This technique will be used in future studies in diatomic and polyatomic molecules.

Another major accomplishment was the successful construction of a non-collinear optical parametric amplifier (NOPA). The NOPA is pumped by a custom modified regenerative amplifier laser (50 femtosecond, $800 \mathrm{~nm}$ pulses) and produces tunable radiation from 480 $750 \mathrm{~nm}$ (signal). Typical output parameters are 15-17 $\mu \mathrm{J} /$ pulse, $\approx 20 \mathrm{~nm}$ of bandwidth (at 530 $\mathrm{nm}$ ) and sub 40 femtosecond pulses. The large bandwidth will ensure the excitation of multimode vibrational wavepackets in polyatomics where large inter-vibrational mode separations are common.

Initial experiments have shown that we can successfully excite vibrational wavepacket motion in jet-cooled $\mathrm{Br}_{2}$ using the NOPA. The experiments involve exciting a superposition of vibrational states in the electronic $\mathrm{B}$-state of $\mathrm{Br}_{2}$ and probing the dynamics using timeresolved photoelectron spectroscopy (TR-PES). The TR-PES method reveals the large spectral bandwidth that is attainable with the NOPA as it is transferred to a broad range of vibrational states. Current efforts are underway to implement stimulated emission pumping (SEP) in which the vibrationally excited molecule is pumped back down to the electronic ground state with a time-delayed 'dump' pulse, thereby launching highly excited ground state vibrational motion. This work will be extended to the first polyatomic system, $\mathrm{CH}_{3} \mathrm{I}$ in which the electronically excited state is a dissociative state (C-I dissociation). 
Spectroscopy and Dynamics of Pure and Doped Helium Nanodroplets Principal Investigator: Daniel M. Neumark

\section{Project Description:}

This project will perform photoionization and photoelectron spectroscopy experiments on atoms and molecules absorbed onto large helium droplets, $\sim 10^{4}$ atoms, in order to understand how these fundamental and well understood gas phase processes are altered in the environment of a helium droplet. A helium droplet source will be constructed and used on Endstation 3 of the Chemical Dynamics Beamline at the Advanced Light Source (ALS). Photoionization mass spectrometry and photoelectron spectroscopy experiments will be performed using the high-throughput vacuum ultraviolet monochromater on this endstation.

\section{Accomplishments:}

During the past year we have carried out photoionization experiments on pure $\mathrm{He}$ droplets on the Chemical Dynamics Beamline at the Advanced Light Source, investigating how the photoelectron spectra evolve as a function of droplet size at photon energies above and below the ionization potential (IP) of atomic He, $24.6 \mathrm{eV}$. While spectra below the IP are dominated by extremely low energy electrons, those above the IP are very different, showing electron signal at higher kinetic energy than those photoelectrons produced from atomic He. The higher energy electrons are attributed to direct photoionization in which small He cluster cations are formed that are embedded within the much larger droplet; vertical photoionization accesses the long-range attractive wells of these cation clusters, resulting in photoelectrons with higher electron kinetic energy. We have also carried out photoionization experiments on He droplets doped with $\mathrm{NO}$ and $\mathrm{SF}_{6}$ using both synchrotron radiation and laser-based resonant multiphoton ionization. 
Soft X-ray Spectroscopy of Liquid Surfaces

Principal Investigators: Richard Saykally and David Shuh

\section{Project Description}

We propose to further develop and apply the technology of x-ray spectroscopy of liquid microjets for the study of liquid surface chemistry, specifically exploiting the capabilities of the new Molecular Environmental Science (MES) Beamline 11.0.2. Liquid microjets allow the detection and subsequent analysis of electrons and ions induced by core level excitation of a liquid interface which, if combined with the unique optical capabilities of the new MES Beamline, will allow the study of liquid surfaces in unprecedented detail. Our goal is to apply this technique to the study of complex systems encountered in many diverse fields such as heterogeneous chemistry, biology, and environmental chemistry.

X-ray absorption fine structure (XAFS) spectroscopy is a powerful tool for probing the electronic and geometric properties of interfaces. Until recently it has been prohibitive to apply this approach to the study of volatile liquid surfaces (e.g., water) due to the high equilibrium vapor pressure of these liquids ( 20 torr), which is incompatible with the ultra high vacuum environment necessary for soft x-ray spectroscopy. By employing liquid microjet technology, we have been able to transcend this technical difficulty and achieve windowless coupling of volatile liquids to the soft X-ray beamlines of the Advanced Light Source. Liquid jets provide unique targets, affording the opportunity to examine liquid surface chemistry with near-edge XAFS and extended-XAFS techniques.

\section{Accomplishments}

Our recent investigations have focused on three separate areas of interest. The first of these is the temperature dependence of volatile liquids. A strong temperature dependence of oxygen Kedge x-ray absorption fine structure features was observed for supercooled and normal liquid water droplets prepared from the breakup of a liquid microjet. Analysis of the data over the temperature range 251 to 288 kelvin $\left(-22^{\circ}\right.$ to $\left.+15^{\circ} \mathrm{C}\right)$ yields a value of $1.5 \pm 0.5$ kilocalories per mole for the average thermal energy required to effect an observable rearrangement between the fully coordinated ("ice-like") and distorted ("broken-donor") local hydrogen-bonding configurations responsible for the pre-edge and post-edge features, respectively. This energy equals the latent heat of melting of ice with hexagonal symmetry (ice Ih) and is consistent with the distribution of hydrogen bond strengths obtained for the "overstructured" ST2 model of water.

We have also completed studies on a variety of amino acid solutions. Specifically, we have measured the $\mathrm{pH}$ dependence of the NEXAFS spectrum of aqueous glycine. By comparing the aqueous spectra to those of the gas and solid phase, we are able to assign all three $(\mathrm{C}, \mathrm{N}, \mathrm{O})$ elemental edges. Only minor perturbations are observed in the carbon edge and the experimentally accessible portion of the oxygen edge. We find that that the position of the nitrogen main edge is determined by the charge state of the molecule, and that the presence or absence of pre-edge features is determined by the hydration state of the molecule. Specifically, we find that the nitrogen terminus is completely associated to the solvent at moderate and low $\mathrm{pH}$ values, but is in an acceptor only configuration at high $\mathrm{pH}$.

Finally, we have studied the effect of the addition of alkyl halides on the NEXAFS spectrum of liquid water. We observe a dramatic decrease in the post-edge and an increase in both the pre-edge and main-edge with increasing salt concentration. These effects are anion specific. The observed spectral changes are primarily caused by the strong perturbative effect of the ionic species on the unoccupied molecular orbitals of the solvent molecules, and only secondarily by geometric reordering. This is in contrast to the temperature dependent changes, which result from rearrangement of the hydrogen-bonding network. 
Scientific Investigations and Technique Development of Wet Spectroscopy, High Pressure

Photoelectron Spectroscopy, and STXM for Molecular Environmental Science

Principal Investigator(s): David K. Shuh and Mary K. Gilles

Project Description

Three end stations at the Advanced Light Source-Molecular Environmental Science (ALSMES) beamline are the wet spectroscopy, high pressure photoelectron spectroscopy, and the scanning transmission x-ray microscope (STXM) systems. The external experimental chambers are the soft $\mathrm{x}$-ray end station for environmental research, the wet spectroscopy surface science system, and the microjet system for the investigation of liquid surfaces. One common experimental theme and capability of all the end stations that we will apply is the ability to perform investigations of interfacial systems in the presence of water or under ambient environments. A second theme is the use of high spatial resolution in combination with spectroscopy to extract chemical information from the micro- and nano- scales and to use this information about distribution to further understand macroscopic chemical behavior.

Research topics to be investigated are broadly defined in a range of scientific areas that include surface science, catalysis, materials chemistry, interaction of biomaterials and organics at interfaces, and the environmental science of a wide range of particulates. Similarly, there are numerous opportunities to investigate the interfacial chemistry of several gas phase and liquid species, including water and small molecules, at the surfaces of metal oxides and sulfides. The capabilities of the end stations will be developed and optimized for specific experimental studies in the aforementioned areas.

Accomplishments

Initial efforts of focused on the development of experimental capabilities at the ALS-MES Beamline 11.0.2. Two major accomplishments during the last year were the installation of a novel, custom-designed magnetic shield for the wet electron spectrometer end station and work on the development of two specialized end stations. The ALS-MES sample preparation chemistry laboratory on the mezzanine of the ALS, which serves a vital role in the overall scientific mission of the beamline, was fully outfitted and maintained.

New collaborative research efforts were initiated with several groups centering around the unique MES capabilities of the ALS and the ALS-MES for spectromicroscopy of particles in the soft $\mathrm{x}$-ray region. Scanning transmission $\mathrm{x}$-ray microscope (STXM) investigations were conducted on carbonaceous aerosols with L. Russell (Scripps Institute of Oceanography, U.C. San Diego); laser annealing of soot with H. Michelsen (Sandia National Laboratory, CA); and lastly on carbonaceous star dust, meteorites, and interstellar dust particles with A. Westphal/A. Butterworth (Space Sciences Laboratory, U.C. Berkeley). There has been great interest in developing a reaction cell for STXM experiments. An in-situ reactor cell, designed and fabricated earlier in this project, has been successfully used several times for STXM catalysis experiments on powdered catalyst materials with I. Drake/A. Bell/S. Leone (U.C. Berkeley). Another thrust has been to use the STXM to image and spectroscopically characterize polymer patterned photoresists with W. Hinsberg (IBM-Alamaden) and S. Leone (U.C. Berkeley). The results from these novel spectromicroscopy investigations are in varying stages of the scientific publication process, with several already being accepted or published. 


\title{
Computing Sciences
}

LB02012

\author{
Project Title: The Experimental Mathematician's Toolkit \\ Principal Investigators: David H. Bailey, Xiaoye S. Li
}

\section{Project Description:}

This project seeks to develop a set of computational tools to permit mathematicians and physicists to perform many of the high-precision numerical computations now being done in the emerging field of experimental mathematics. In particular, we seek tools to calculate various mathematical expressions to hundreds (and sometimes thousands) of digit accuracy, and then to determine, by means of integer relation techniques, whether the calculated values are given by some simple formula.

Some of these tools, such as high-precision numerical libraries and high-level language software, have been developed by the investigators and others in previous work. In other cases, prototype computer programs are working, but further enhancements are needed. The next step is to make these facilities available to mathematicians and physicists as interactive and web-based tools, thus eliminating the need for sophisticated computer programming. We believe that tools such as these will facilitate numerous new results in the arena of experimental math.

Accomplishments:

One achievement is the development of computer programs to evaluate a broad range of definite integrals to unprecedented accuracy (up to 2000-digit precision), including integrals of functions with singularities at endpoints. Similar programs have also been developed and tested for 2-D integrals, and parallel computer versions are also working.

A second achievement is an interactive program, known as the Experimental Mathematician's Toolkit, which combines a high-precision computational system (up to 1000 digits) with numerous additional facilities such as integrals, infinite series sums, polynomial roots and integer relation detection. In addition, this tool is now available as a web-based facility: https://nebula.lbl.gov/cgi-bin/login.py.

A detailed description of the computational techniques employed are described in several papers and in two new books, published in 2004, co-authored by one of the investigators (DHB). A review of the two books, published in Math Reviews begins, "Let me cut to the chase: every mathematics library requires a copy of this book and its companion volume." The full review is available at: http://www.cs.dal.ca/ jborwein/mr-mbye.pdf.

This work has received significant press attention, including feature articles in Scientific American (May 2003) and Science News (Apr 24, 2004). The Science News article is subtitled, "Computer Experiments Are Transforming Mathematics." 


\title{
LB02013
}

\author{
Infrastructure for Improving Protein Structure Prediction in Computational Biology \\ Principal Investigators: Silvia Crivelli and Wes Bethel
}

Project Description

One of the grand challenges in modern science is the prediction of the three-dimensional structure of a protein given its primary sequence of amino acids. There are two distinct approaches to tackle the protein structure prediction problem: knowledge-based methods that depend on the presence of homologous sequences in databases and physics-based methods that rely on the underlying physical principles. Although the physics-based methods are extremely important to find genuine folds, they are also extremely expensive. Therefore, knowledge-based methods become a valid alternative when there is some homology. However, in most cases only fragments of the proteins are similar, therefore, the practical applicability of homology methods is limited.

During our first two years of LDRD effort, we developed ProteinShop, an interactive graphical environment for creating and manipulating protein structures with the following goals: 1) to create the initial configurations for a protein structure prediction method and 2) to develop a new, knowledge-based method for protein structure prediction. We achieved our first goal during the first two years. In fact, ProteinShop has been instrumental in our participation in CASP5 -a worldwide competition for the assessment of protein structure prediction methods held in 2002allowing our group to triplicate the number of targets submitted and to predict targets with sizes and topologies that were not possible before. Moreover, many groups who competed in CASP5 began using this tool for their own methods in preparation for CASP6. ProteinShop has been downloaded by hundreds of groups worldwide.

\section{Accomplishments}

Our most significant accomplishment during FY04 has been our goal \#2. We developed a new methodology for protein structure prediction that uses ProteinShop not only to create initial configurations based on known fragments but also to steer the global optimization solver. The new method differs from our previous one in that 1) it incorporates knowledge from known proteins to design the initial configurations and 2) it combines filtering techniques with a physics-based energy function to enhance the structure discrimination ability and the performance of the method. Our new method is based on the observation that even if the fold recognition servers provide only limited and incomplete folding information for the targets in the new folds category, such information may still be valuable for guiding the global optimization process to find the native conformation. We have tested the new methodology in the recent CASP6 (summer 2004). The results will be known shortly.

Our final release, ProteinShop version 3.0, is another significant accomplishment. This version adds important new capabilities to the original one. The new release includes features to perform energy visualization simultaneously with manipulation, fully automatic creation of low-energy beta-sheet configurations, local minimizations, multi-protein and multi-domain visualization, protein alignment and root mean square deviation, and different protein rendering styles. Energy visualization highlights regions of extraordinarily high energy, providing hints for where manipulations may be most effective or for how the energy and the gradient change during local optimization. Visualization is a crucial part of the feedback process needed to enable human interaction and human interaction is crucial to accelerate time to solution. We plan to apply the same ideas to expand ProteinShop to such areas as RNA structure prediction, protein-protein docking, and drug design. 


\title{
New Machine Learning and Data Mining Methods for Genomics and Climate Data Analysis
}

\section{Project Summary.}

\author{
Principal Investigator: Chris Ding
}

We propose to continue develop new machine learning and data mining methods for discovering novel patterns in scientific datasets. The applications will focus on genomics and climate datasets. In genomics, we develop methods for protein complex prediction and gene regulatory network prediction. For climate datasets, we will develop methodology for better capture of the essential characteristics of 2D and 3D fields.

\section{Accomplishments.}

(1) Protein Interaction. Large amount of Experimental data on protein interactions have been produced, such as two-hybrid data and TAP-MS data. We have developed a unified representation of protein complex data collected by TAP-MS experiments in yeast using a bipartite graph model. This allows for weighting of connections between proteins shared in more than one complex, as well as addressing the higher level organization that occurs when the network is viewed as consisting of protein complexes that share components. W were able to identify clusters in the protein-protein network (complexes) and the complex-complex network (supercomplexes) and annotate them using the Gene Ontology (publication [1])

(2) Discovering novel functional RNAs. We developed a new method using positive only data samples to learn RNA genes from DNA sequences. The methods breaks DNA sequence into 80-nucleotides windows and establish a set of feature vectors. From the feature vectors and the known RNA examples, we predict new RNA genes using a Support Vector Machine methods. The method is found competitive to existing methods (see publication [2]).

(3) Principal Component Analysis (PCA) is one of the most widely used multivariate statistical methods. PCA is developed in 1930's. One of the most widely used data clustering method is K-means method, which iteratively improve the data prototypes (cluster centroids) minimizing an squared error function. K-means is developed in 1960's. We recently proved rigorously that PCA is equivalent to K-means, i.e., the optimal solution of K-means clustering is given by the principal components (see publication [3]). (4) We develop clustering methods to discover meaningful patterns in experimental datasets. These methods use eigenvectors of the Laplacian matrix of the similarity graph. They have many desirable mathematical properties and easily computable. We proved the optimality of the ordering of the nodes of the graph; we introduced a cluster-crossing metric to map the clustering problem into a one-dimensional problem which can then be solved effectively. (see publication [4]).

(5) Developed a fast Bayesian network structure learning methodology for detecting gene regulatory networks. Our method uses a new candidate graph approach and then deduct the best acyclic directed graph from it. We use new cycle elimination heuristics and structural perturbations to test local stability and improvements. (see Publication [6]) (6) We developed a new 2-dimensioanl Singular Value Decomposition for a set of 2D objects such as images or weather maps, using principal eigenvectors of row-row and column-column covariance matrices. This 2D-SVD preserves the $2 \mathrm{D}$ characteristics of the original data, and thus provides a better low-rank approximations than standard SVD. (see publication [7]) 


\section{Project Description}

\section{Interactive Visualization Methods for Exploration and} Comparison of Multi-billion Base Pair Sequence Data Principal Investigator(s): Bernd Hamann

SNPs Visualization. Common severe congenital malformations (such as congenital heart defects) are the result of complex interplays between both genetic and environment factors. The recent completion of a single version of the human genome has now provided the substrates for direct comparison of individuals in both health and disease. With today's resources, it is possible to re-sequence a large set of appropriate candidate genes in individuals with a given disease to screen for causative mutations. Such an approach is beginning to prove fruitful in the area of cancer genetics and is likely to contribute to our understanding of gene mutations responsible for sporadic forms of congenital disorders. A single nucleotide substitution is called a single nucleotide polymorphism or "SNP." SNPs are common but minute variations that occur in human DNA at a frequency of one every 1000 bases. Comparison of re-sequenced genes of disease-carrying individuals to unaffected controls gives SNPs data that needs to be analyzed to distinguish common SNPs from causative mutations. As SNP data sets have grown larger with increasing number of individuals and genes being re-sequenced, it has become essential to provide interactive visual exploration tools for this kind of data. We have been developing a SNPs visualization tool that aids a scientist in the discovery process of severe mutations in disease-related genes from re-sequencing data.

3D Gene Expression Visualization. It is intrinsically difficult to visualize complex gene expression patterns and morphology in three dimensions, especially at cellular resolution. As part of the Berkeley Drosophila Transcription Network Project (BDTNP), we have begun with the development of methods to overcome this challenge by using images of whole Drosophila embryos.

\section{Accomplishments}

We have developed a SNPs visualization tool that classifies SNPs and maps SNPs to gene structure, aiding biologists in the discovery process of severe mutations in disease-related genes from re-sequencing data. We plan to make this tool available with a suite of SNPs analysis tools developed by "SeattleSNPs," a collaboration between the University of Washington and the Fred Hutchinson Cancer Research Center, having as a goal to discover and model the associations between single nucleotide sequence differences in the genes and pathways that underlie inflammatory responses in humans.

In the context of gene expression analysis via imaging and interactive visualization, we have adapted volume-rendering methods to multi-channel confocal microscopy data, every single channel of an "image stack" is rendered independently by mapping brightness information to color and transparency information. Resulting colors are blended using user-specified weights, supporting seamless blending between channels, including the possibility to show all three channels simultaneously. Our volume visualization prototype has been combined with user interaction tools to support quantitative determination of the accuracy of the BDTNP's nuclear segmentation methods. Nuclei are rendered to include information obtained from a nuclear segmentation mask. It is possible to select individual nuclei interactively and identify falsely segmented objects. This work has already yielded significant improvements in segmentation accuracy.

Finally, we are developing a visualization tool for 3D gene expression data in the form of matrices that describe nuclear positions and their associated gene expression levels. 


\section{Optimizing Genomic Data Storage for Wide Accessibility \\ Principal Investigators: Nancy Meyer, Damian Hazen, Jonathan Carter}

\section{Project Description}

The purpose of this project is to improve and enhance access to the data generated at the DOE Joint Genome Institute (JGI) Production Genomic Facility (PGF) for the genomic research community by developing optimized techniques for the storage and distribution of genomic data. The PGF at JGI is one of the world's largest public DNA sequencing facilities. The PGF produces 2 million files per month of trace data, 100 large assembled projects per month, and several very large assembled projects per year. The archival management scheme that we are developing in collaboration with JGI will ensure a scalable solution (billions of files), optimize the placement of genomic data for referencing and retrieval, allow partial and/or whole retrieval of data, and enhance the ability to organize and cluster the data by incorporating reference models being developed by JGI.

There is a growing need for the PGF to archive its data to the current and planned large-capacity NERSC storage systems for distribution to the research community. The storage systems are robust and available 24 hours a day, 7 days a week. The systems are scalable, configured for high availability, and tuned for good network bandwidth. NERSC has high-quality, high bandwidth connectivity to DOE laboratories and major universities provided by ESnet. NERSC is a collaboratory development site for the HPSS storage system.

\section{Accomplishments}

We are currently archiving all the raw trace data produced by the PGF and making this data available on line to researchers at JGI. We have tuned wide-area network transfers between JGI and NERSC for optimal GigE performance. Utilities have been installed at JGI to efficently transfer data to and from NERSC and to create large aggregated collections of data on the NERSC storage system. This archival technique will scale over time and currently allows the packaging of millions of data files into one large archive file. This archival technique also optimizes the placement of genomic data for retrieval. Access to any file in the storage aggregation results in the efficient (MBs/second) streaming of the whole aggregation from the dense media to fast access media, where millions of files can now be immediately accessed optimally. An index of the data in the aggregated collection is kept and metadata operations that reference the data use this index which allows efficient referencing of the data without data movement. Whole and/or partial retrieval of data is supported: files can be extracted individually, or the large aggregation can be transferred, supporting multiple uses of the same data by different applications and differing research requirements. 


\section{Parallel Methods for Robust Optimization and Uncertainty Quantification Principal Investigators: Juan C. Meza, Ricardo Oliva, Silvia Crivelli}

\section{Project Description}

Optimization of functions derived from the modeling and simulation of some physical process constitutes an important class of problems in many scientific applications. Often, the computer simulation entails the solution of a system of nonlinear partial differential equations (PDE) in two or three dimensions. Other applications include particle dynamics simulations, problems in chemical kinetics, and PDE constrained optimization problems. The main characteristic of these types of problems is that the function evaluation is computationally expensive and dominates the total cost of the optimization problem. Depending on the nature of the application and the solution method employed, there can also be noise associated with the evaluation of the objective function and the experimental data. In many applications it is also the case that there are uncertainties in both the model parameters and the experimental data. In these cases it is desirable that the optimal solution be robust in the sense that small changes in the model parameters or data do not generate large perturbations in the optimal solution. Uncertainty quantification has also received a great deal of attention recently as a potential validation tool for large multi-scale, multi-physics application codes.

This research focuses on two major areas: 1) the development of parallel algorithms for robust optimization under uncertainty and 2) the development of optimization methods for large-scale problems.

\section{Accomplishments}

We have developed a new methodology for protein structure prediction that uses ProteinShop not only to create initial configurations based on known fragments but also to steer the global optimization solver. The method uses a combination of global optimizations in subspaces of the dihedral angles space and local optimizations in the full dimensional space to find the protein configuration with the lowest energy. A crucial component of the protein prediction methodology is an accurate energy model that discriminates good folds from misfolds. An important accomplishment of this effort has been the upgrade of our energy code to be consistent with AMBER7 with modified parameters and the coupling of that code with ProteinShop. The modified parameters are designed to improve the discriminatory ability of the energy function by enforcing the formation of hydrogen bonds and beta-sheets. We have tested our method during the recent CASP6 worldwide competition for the assessment of protein structure prediction methods, and we have observed that our optimized structures retain most of the original hydrogen bonds, especially those occurring in beta-sheets. Furthermore, the coupling of the new energy model with ProteinShop has led to the development of an energy visualization feature, which permits us to highlight regions of high energy to guide the steering process.

Another important accomplishment has been the integration of OPT ++ to ProteinShop for the local optimization of selected protein structures. Structures created or manipulated with ProteinShop can also be locally minimized with ProteinShop. The user can take advantage of the energy visualization and optimization features to monitor the energy and gradient changes during the folding process. In addition, we have investigated a divide-and-conquer approach to speed-up the local optimization process, in which we divide the polypeptide chain into clusters of segments that are optimized in parallel and later combined for refinements. The preliminary results are very encouraging and robust with regard to how the chain is split. 


\section{Combinatorial Algorithms in Scientific Computing}

Ali Pinar

Project Description: Combinatorial algorithms have long played an important role in many scientific computing applications. Their growing importance of combinatorial algorithms in emerging applications like computational biology and scientific data mining calls for development of a high performance library for combinatorial algorithms. Building such a library requires a new structure for combinatorial algorithms research that enables fast implementation of new algorithms, as well as new algorithms that are amenable for high performance computing. The objective of this project is not only producing solutions to some fundamental problems in scientific computing, but also designing a research structure for combinatorial scientific computing, which can accommodate computationally efficient libraries for a wide variety of applications and techniques.

Accomplishments: We have investigated combinatorial problems arising in parallel computing, sparse matrix computations, and scientific data management. Our work has not only produced solutions to important problems, but also helped us to design a research structure towards high performance libraries.

Efficient utilization of parallel computers require balancing computational load and communication algorithms among processors, both of which are combinatorial problems. We studied exploiting flexibly assignable tasks (tasks that can be assigned to one of many processors) to improve load balancing, one-dimensional partitioning of workload arrays, and interprocessor communication with memory constraints.

Sparse matrix computations are commonly associated with combinatorial algorithms. We have studied the problem of finding nonoverlapping dense blocks of a matrix to improve memory performance of sparse matrix computations. We have also studied finding a sparse null-space basis for a sparse matrix by cleverly choosing a basis.

With the advances in technology, scientific experiments generate massive volumes of data everyday, making storage and indexing an important problem. We studied data reorganization to sizes of bitmap tables, which are widely used in scientific databases. We showed the relation between this problem and the traveling salesman problem and proposed Gray-code ordering that reduces the bitmap table size by a factor of 7 . 
Advanced Computational Methods for Photon- Molecule Collision Processes

Principal Investigators: Thomas N. Rescigno and C. William McCurdy

Project Description

Sophisticated calculations of collisional ionization processes involving two electrons in the continuum have so far been limited to light atomic targets. For analogous processes involving simple molecular targets, no first-principles calculations have appeared to date. The purpose of this project is to develop advanced computational methods for studying certain photon-molecule collision processes that are currently beyond the grasp of first principles methods. We will develop grid-based ab initio methods for studying double photoionization (one photon in, two electrons out) of atoms and small molecules. These theoretical methods will assist in the interpretation of current experiments at the ALS on double photoionization of $\mathrm{D}_{2}$ and provide a predictive tool for motivating new experiments.

The method of exterior complex scaling (ECS will form the basis of our approach. This method provides a path to the computation of scattering wave functions without having to explicitly impose detailed asymptotic boundary conditions. A variety of grid-based techniques will be employed in the ECS implementation. We will also extend our studies to small polyatomic targets by combining grid-based methods with modern quantum chemical techniques.

\section{Accomplishments}

We have succeeded in making rapid progress on the first phase of the proposal, which was to demonstrate that the ECS method is a viable aproach for studying double photoionization. In collaboration with Prof. Fernando Martin in Madrid, we have successfully carried out an ECS adaption for atomic double photoionization using B-splines. Three papers have resulted from this preliminary work, one describing the new theoretical methods, another presenting detailed and essentially exact differential cross sections for double photoionization of helium and a third paper on symmetrized amplitudes for double photoionization of helium has been accepted for publication.

We have also made significant progress on the molecular front, using exterior scaled Bsplines to carry out what will be the first ab initio calculation of double photoionization of $\mathrm{H}_{2}$. These early calculations, which use a single-center expansion, are showing that the basic ideas are sound, but that it may be difficult to obtain fully converged differential cross sections with a B-spline implementation. We are therefore also working on replacing B-splines with a more efficient finite-element/discrete variable representation (DVR) approach we have developed. The DVR will eliminate most of the time associated with computing two-electron matrix elements since they have a simple diagonal representation in the DVR.

The DVR approach does not eliminate the need for single-center expansions, which quickly become impractical for larger molecules. We are therefore pursuing a parallel effort that combines a more traditional molecular orbital approach with exterior-scaled DVR functions. This novel approach will eliminate the need for slowly convergent single-center expansions at the cost of increased complexity in evaluating two-electron matrix elements. The basic ideas, in work which formed the basis of a UC Berkeley Masters thesis, have been successfully tested in the computation of one-electron molecular continuum wave functions. We believe that this hybrid development will pave the way for applications to polyatomic targets. 


\section{Evaluation of Computer Architecture Alternatives Principal Investigators: Paul Hargrove and Katherine Yelick}

\section{Project Description}

The goal of this project is to evaluate the technology building blocks of high performance machines for future acquisitions at LBNL, and across DOE, by examining both established and emerging technologies for processors, memory systems and interconnects from industry, academia and government laboratories. This work will provide DOE with insight into emerging designs and their potential effects on next-generation supercomputing architectures and the applications that run on them. It will provide a mechanism for evaluating future designs through collaboration with industry, and may lead to the inclusion of specific system features in those designs.

Our approach is to use a combination of microbenchmarks, application kernel benchmarks, and performance models to measure the benefits and limitations of emerging architectural technologies. The application kernel benchmarks help identify the need for new algorithms for a given architecture, and allow us to explore an entire class of problems. The microbenchmarks isolate particular aspects of the hardware that prove to be a bottleneck, and thereby enable studies of variations on a particular hardware implementation. Finally, we will work with new and existing performance models to predict the performance on machines that do not yet exist.

\section{Accomplishments}

We designed a microbenchmark called the Stencil Probe to emulate grid-based codes and used it to identify memory system bottlenecks on current microprocessors and to measure the effectiveness of cache optimizations. We determined that cache tiling techniques designed for 2D grids that were effective in the past are less effective today because of increasing cache size, the trend towards 3D computations, and the importance of prefetching. Cache tiling techniques decrease cache misses, but often increase the rate of non-contiguous memory accesses. We quantified this trade-off using a simpler S-DAXPY benchmark, which measures the effectiveness of prefetching on unit and non-unit stride accesses.

We also continued work on the Sqmat benchmark, a probe with parameters for computational intensity, regularity of memory access, and working set size. We showed that this benchmark matches characteristics of the serial HPC Challenge benchmarks, while also allowing for a more continuous evaluation of temporal and spatial locality. We began extending the idea of architectural probes to a parallel setting with a preliminary code that measures network performance while varying the number of nodes and degree of communication.

In addition, we continued work on evaluation of data parallel architectures, such as vector, streaming, and SIMD processors, especially focusing on VIRAM. These architectures differ in their register file organization and associated data movement instructions between registers. VIRAM has a single register file and a limited set of permutations. This project demonstrated the use of advanced compiler technology for automatically generating register permutation instructions; for general data movement patterns for sorting or Fast Fourier Transforms, the compiler generates code that is competitive with hand-optimized assembly.

Lastly, we have participated in discussions with several HPC vendors, informing them of our application requirements, and suggesting design choices that would improve performance for DOE codes. 


\section{Earth Sciences Division}

LB03008

Application of real-time PCR with reverse transcription for quantification of specific microbial activity in complex communities

Principle Investigators: Lisa Alvarez-Cohen and Terry Hazen

Project Description

The purpose of this project is to develop and apply culture-independent molecular approaches for characterizing microbial communities capable of degrading chlorinated solvents in subsurface aquifers. These approaches will be used to concurrently quantify 1) the occurrence of specific dehalogenating microorganisms, 2) genes associated with the dehalogenation reaction, and 3) the expression of the dehalogenase genes. Correlating measured dechlorination activity with molecular measurements in laboratory pure cultures, laboratory enrichment cultures, and field samples will allow us to determine the important factors that affect gene expression, evaluate the contribution of the identified dechlorinator to the overall degradation reaction in the environment, and determine whether the functional gene is present in species other than the known degrader.

Our approach is to apply quantitative real-time PCR (qPCR) and reverse transcription to quantify the occurrence and expression of dehalogenating species and genes in laboratory enrichments and field samples under varying conditions. Initial nucleic acid targets for this approach were the Dehalococcoides $16 \mathrm{~S}$ rDNA, the tce $A$ dehalogenation gene, and tce $A$ gene mRNA. The recently identified reductase genes, $v c r A$ and $b v c A$, have also been included.

Accomplishments

First, several pure and mixed dechlorinating microbial cultures have been examined for the presence of specific $16 \mathrm{~S}$, $t c e A, v c r A$, and $b v c A$ genes. The use of a single DNA standard for all four target genes has dramatically increased the accuracy of DNA copy number measurements. In the mixed ANAS enrichment culture, the Dehalococcoides genus 16S copy number agrees well with the copy number sum of tceA and vcrA reductases, suggesting that dechlorination in the ANAS culture is a cooperative effort between two strains of Dehalococcoides. Indeed, when ANAS subcultures were grown to specifically enrich each gene, the ratio of tceA to $v c r A$ varied from $3: 2$ under $t c e A$ favoring conditions to $1: 9$ under $v c r A$-favoring conditions.

Second, the expression of the tce $A$ gene has been quantified under multiple physiological conditions to understand how these conditions might impact dechlorination activity. We find that tce $A$ gene expression increases upon exposure to the physiological substrates TCE, cis-1,2dichloroethene, trans-1,2-dichloroethene, or 1,1-dichloroethene, but not in response to perchloroethene or vinyl chloride. This pattern of expression response does not vary with the total solvent concentration, the concentration of electron donor $\left(\mathrm{H}_{2}\right)$, or the competitive presence of common microbial electron acceptors. Incubation temperature, however, had a substantial effect on tce $A$ expression, with $t c e A$ expression at $30^{\circ} \mathrm{C}>10$-fold higher than at $14^{\circ} \mathrm{C}$.

Third, we have continued measuring the presence of dechlorinating species and reductase genes in groundwater samples from Seal Beach, California, where only partial dechlorination has been observed. This year a Dehalocococcoides-containing culture was injected into the Seal Beach aquifer. Our analysis showed that Dehalococcoides 16S coincided with dechlorination end-products, both in time of appearance and magnitude, across multiple wells over several months.

Success in measuring the tceA expression response to environmental conditions has led to initiation of a genome-wide expression analysis study using Affymetrix microarrays. This technique should identify novel genes involved in dechlorination and thus open even more avenues of study. 


\section{Microbial Controls on Metals in the Environment \\ Principal Investigator: Jillian Banfield}

\section{Project Description}

Microorganisms can largely control the form, distribution, mobility, and toxicity of metals in the environment. Microorganisms can increase the rates at which environmental metal pollution occurs through increasing the rate of mineral dissolution or by altering the speciation of metal ions through organic complexation. Conversely, microorganisms can directly and indirectly decrease the mobility and bioavailability of metals in the environment through metal uptake and sequestration, adsorption of ions onto cellular and extracellular polymers, and by inducing precipitation of metalcontaining minerals.

In the later part of this project we have focused primarily upon microorganisms that populate extremely acidic, metal-contaminated environments. Our approach has been to combine conventional cultivation-based approaches with molecular methods in order to identify the organisms present and to determine the pathways and interactions central to pyrite dissolution and acid mine drainage formation. Central to our approach was partial reconstruction of genomes of dominant microbial community members directly from DNA extracted from environmental samples, avoiding limitations associated methods that depend upon cultivation. We used this approach to identify the molecular pathways involved in community-essential functions such as fixation of carbon and nitrogen and biofilm polymer production, defined the microbial community structure at the strain level, and characterized heterogeneity within populations.

Accomplishments

We partially reconstructed five genomes from a well characterized acid mine drainage biofilm and analyzed the results to provide metabolic insights. The findings were reported in Tyson et al. in Nature. This work was cited by Science, along with a subsequent paper of Venter et al., as one of the top 10 breakthroughs of 2004. The genome of the dominant bacterium has since been assembled into 58 genome fragments (scaffolds) and the dominant (uncultivated) archaeal population into 16 scaffolds (the largest being $\sim 640 \mathrm{~kb}$ ). In addition, we reconstructed a small scaffold from an unknown organism. Based on phylogentic analyses using several genes encoded on the scaffold, it is a novel, deeply branching archaeon. This organism, previously invisible to us, is almost ubiquitous in the system. Function of one gene, a putative arsenate reductase, has been proven via complementation in a deficient $E$. coli strain. The eukaryotes in the AMD system were documented by molecular methods. A subset of the protists have an alphaproteobacterial endosymbiont.

Using insights from the genomic data, we cultivated the first representative of Leptospirillum group III, showed that it is an iron oxidizing chemoauthotroph, and confirmed its ability to fix nitrogen. The culture is closely related to, but not identical to, the dominant strain sampled in the community genomics study. Analysis of heterogeneity within the dominant bacterial populations showed that they are near-clonal. In contrast, we documented extensive recombination within archaeal populations and showed that they are characterized by mosaic genome structure. On going analyses are evaluating heterogeneity in gene content and gene sequence and targeting genes inferred to be under selection for further analysis. 
Autonomous Sensors for Ocean Dissolved Organic Matter

Principal Investigator (s) James Bishop, Bill Edwards (FY04), Michael Vestel (FY04)

\section{Project Description}

Dissolved organic carbon (DOC) in seawater constitutes a large, dynamic, yet poorly quantified reservoir of carbon in the oceans. Changes in the concentration of DOC reflect transformation of carbon between inorganic and organic, and between dissolved and particulate species. DOC changes may thus be a critical yet independent measure of changes in the biological pump in the ocean or of changing carbon sequestration in the ocean. Here, we propose development of a novel autonomous ocean-profiling sensor for dissolved organic carbon in seawater. Such a sensor will allow -- for the first time -- fully autonomous quantification of all products of marine photosynthesis on the time scales of marine photosynthesis. Our effort supports current national initiatives for carbon prediction, management, and assessment.

Marine biofouling begins with adsorption of an organic film on a fresh surface. The process initiates on timescales of seconds. Our sensor concept is to present to the environment a pre-cleaned organic-free surface purposefully engineered for non-specific adsorption of dissolved organic matter from seawater. The developing organic film will be monitored for seconds, the surface will be cleaned and the measurement process repeated. This multi-year LDRD project aimed to explore mass and electrochemical approaches to the measurement of $\mathrm{DOC}$ and to investigate surface cleaning.

\section{Accomplishments}

We have established laboratory methodology for accurate and precise quantification of DOC in oceanographic samples. We tested the concept of a mass-sensitive detector with integrated electrodes for electrochemical cleaning. For this we used a Quartz Crystal Microbalance (QCM) and potentiostat controlled cleaning electrodes. Adsorption/cleaning experiments were performed in a beaker using seawater. Electrochemical cleaning restored the sensor to it's original mass, but we could not determine the nature of adsorbed matter.

The QCM apparatus was thermally stabilized and modified for measurement under conditions of flow. The solution matrix was simplified. Adsorption/desorption experiments were performed by alternating mixtures of $2 \mathrm{mM}$ to $14 \mathrm{mM}$ sucrose $\left(\mathrm{C}_{12} \mathrm{H}_{22} \mathrm{O}_{11}\right)$ in buffered $0.1 \mathrm{M}$ $\mathrm{NaCl}$ with sucrose-free buffered $\mathrm{NaCl}$. We were clearly able to see a proportional response to the added sugar. The Detection Limit was estimated to be $\sim 0.5 \mathrm{mM}$ sucrose $(6 \mathrm{mM} \mathrm{C})$.

We know that electrochemical cleaning is possible and that we could detect clear signals from the adsorption of the sucrose. The QCM proved to be extremely temperature and pressure sensitive. The QCM experiments thus demonstrated that the mass sensing approach is unlikely to be successful in ocean profiling applications. Sensitivity is at least 3 orders of magnitude above those required to measure DOC at oceanic levels $(0.040-0.30 \mathrm{mM} \mathrm{C})$ and at desired $0.0005 \mathrm{mM}$ C precision.

The project is now investigating electrochemical / molecular methods for detection. For this reason, FY04 team members Bill Edwards and Michael Vestel have been replaced by Paul Luke and Mark Ammon. 
Principal Investigator: Bo Bodvarsson

\section{Project Description}

We will conduct pilot studies that are in part motivated by questions raised from discoveries from other solar system objects that can be conducted in Earth laboratories and that address on a specific level the evolution of the Earth's atmosphere and its chemistry, as well as the climate, thermal, and geological history of the Earth.

Accomplishments

Mineral Resource Perspective on Semi-conductor Photo-Voltaic Materials:

The extensive array of known semi-conducting materials from an earth resources perspective as well as their photo-receptor properties shows a number of non-isometric very abundant materials that well warrant investigation. Two possible types of layered semi-conductors were studied. $\mathrm{CuFeS}_{2}$ is one with a basal unit cell of about 5.3 Angstroms. A possible layer sequence to consider would be $\mathrm{CuFeS}_{2} / \mathrm{Si} / \mathrm{AlP}$. $\mathrm{CuFeS}_{2}$ is the mineral chalcopyrite, the most common copper ore mineral mined on earth and AlP is a common product of producing fertilizer from phosphorite and used as an insecticide. Different minerals on other planets may also be promising. Characterization of Nitrous Oxide Isotopologues Emitted from Agricultural Ecosystems:

We have analyzed the complete nitrogen and oxygen isotope compositions of $\mathrm{N}_{2} \mathrm{O}$ from whole air samples collected at a wheat site of the Oklahoma DOE ARM-CART region in collaboration with Margaret Torn of LBNL. Dr. Torn has collected and selected flask samples taken from at least two levels $(2 \mathrm{~m}, 4 \mathrm{~m}, 25 \mathrm{~m}$, or $60 \mathrm{~m})$ on a tower every $2 \mathrm{~h}$ to resolve the diurnal variations of $\mathrm{CO}_{2}$ and its isotopic compositions in the convective boundary layer. The results of this work may be useful in predicting conditions on other planets.

Fault Slip from Repeating Micro-earthquakes in the Mendocino Triple Junction (MTJ) Region:

Our initial results and interactions with researchers at U.C. Berkeley helped stimulated complementary research efforts on unusual seismic events at Berkeley, and together these efforts have now shown that all three unusual event types exist in both contrasting regions. Among the most significant (and certainly the most unexpected) of the findings so far has been the discovery of deep non-volcanic tremor activity deep beneath the San Andreas Fault in the vicinity of Cholame, just south of Parkfield, CA. This discovery is to be published on-line in Science Express on 09 December 2004. The results obtained may have implications for other planets.

Mesoscale Meteorology and Hydrologic Response in Sagehen Creek and Independence Creek: An automated weather station was installed on top of Carpenter Ridge, at the Sierra crest above Sagehen Creek Field Station. Design and installation of the station was undertaken by Prof. James Kirchner and field station managers Jeff Brown and Faerthen Felix. The summit weather station includes instruments for measuring solar flux, temperature, relative humidity, wind direction, windspeed, and precipitation. This instrumentation is expected to contribute to LBNL projects in mesoscale atmospheric modeling first for Earth and later possibly other planets..

Modeling Hydrothermal Systems in Regions with Permafrost:

A spatial and temporal correlation of igneous activity and floods on Mars suggests that magma intrusion within frozen ground may lead to catastrophic discharge of groundwater. We have thus been developing numerical models to simulate magma-water interactions on Mars (and Earth) incorporating thermal convection and phase changes. The focus is on two processes: 1) melting of permafrost, and 2) the role of permeability changes in the solidying igneous body. The main goal of the simulations is to provide new constraints on the evolution of the crust and hydrosphere on Mars. 
Development of Biosensors for Endocrine Disrupting Compounds in Agricultural Watersheds

Sharon Borglin, Chris Campbell, Bailey Green, Hoi-Ying Holman, William Stringfellow, \& Allen Grayson

\section{Project Description}

There are two major obstacles limiting the successful management and removal of EDCs in the environment: (1) EDCs are a diverse class of compounds that are present and active in very low concentrations that are difficult to monitor and (2) EDC sources are numerous and many sources are poorly characterized. There is a need to develop improved tools for monitoring EDCs and apply those tools to understanding and managing EDCs in the environment. In response to these problems this project has identified three goals: (1) develop analytical capabilities to characterize the diverse sources of EDCs, (2) characterize and compare EDC from urban and agricultural sources, and (3) evaluate the connection between EDCs and pesticides and to further understand fate and transport.

Our study continues to develop sensors targeting estrogen compounds and surfactants. The sensors will be used to measure EDCs an open agricultural system (the San Joaquin River Basin, $\mathrm{SJRB}$ ) and various treatment systems. The sensors test the hypothesis that changes in discharge rates and water management practices in the SJRB, such as the timing of water releases, would reduce the impact of EDCs to waters of the state. Additionally, the sensors will be applied in WWTPs to demonstrate their usefulness for management and to test the hypothesis that EDC treatment occurs.

\section{Accomplishments}

The biosensors and extraction and analysis techniques continued to be a major focus of the project using ELISA assays. Testing of influent and effluent samples was begun and will continue at three WWTPs to compare the EDC removal efficiency of two different treatment processes and our 2004 results show significant degradation of EDCs in wastewater. An abstract was presented National Groundwater Association conference on Endocrine disruptors in September 2004. The first round of water and sediment samples was collected from the San Joaquin River Basin at important monitoring points included in on-going water quality and the results will be used to pursue further funding.

In FY04, we completed an extensive literature review (see publications) on the various types of available biosensors. Our review concludes that biosensors like the ELISA technique are the most promising and cost effective of the currently available techniques for reliable EDC analysis. A prototype biosensor system was developed to measure cellular response of luminescent microbes (Figure 1). Extensive testing has begun on the system performance including photo detection sensitivity, reagent mixing, and automating processes.

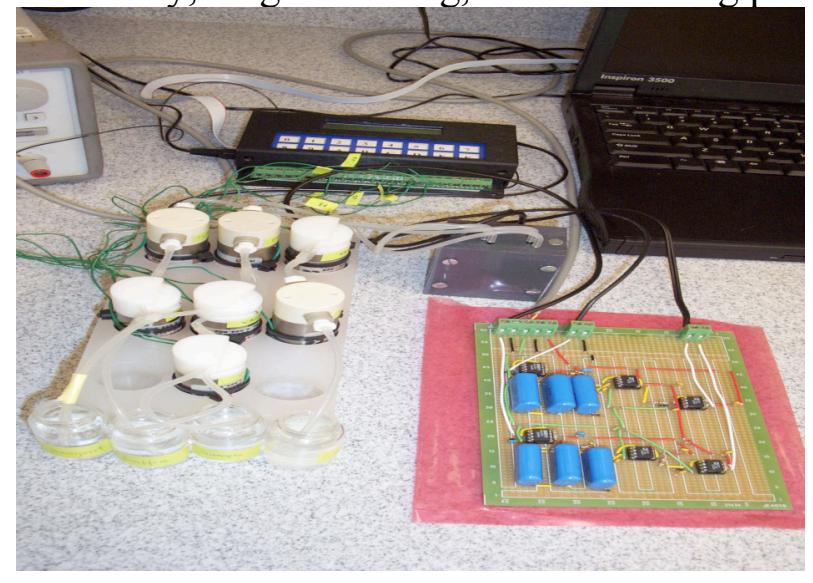

Figure 1: Prototype biosensor system 


\section{The California Water and Energy System: An Approach for Addressing Future Crises}

Principal Investigators: Norman .L. Miller, Nigel T.W. Quinn, Larry L. Dale, Katie Coughlin, Helen He Collaborators: CA Depart. Water Resources, U.S. Bureau of Reclamation, and National Weather Service

\section{Project Description}

The overall purpose of this project is to understand how natural processes and human intervention interact to influence water supply and water quality, and the sensitivity of the system to potential disruptions. We are developing tools that provide reliable quantitative evaluation of risks under different future climate scenarios, and integrate physical, water quality and allocation modeling to quantify water supply reliability under changing conditions.

Our specific goals are: (1) Quantitatively understand the climate drivers impacting mountain front recharge, snowmelt runoff, and net infiltration in the Sierra Nevada and Central Valley. (2) Investigate the sensitivity of the water table to these inputs and to pumping withdrawals. (3) Adapt existing geophysical logging and monitoring techniques as a superior method for characterizing the depth distribution of groundwater contaminants. (4) Develop a regional resource management model to demonstrate economic tradeoffs between agricultural and environmental groundwater pumping, incorporating long-term aquifer degradation. (5) Test electricity data to better characterize groundwater pumping in the Central Valley, and develop a groundwater model to better forecast electricity and groundwater use. (6) Determine the extent to which existing planning models can be used to investigate system response under different hydrological conditions, and whether the mathematical approximations can be separated from the effect of data uncertainty.

Two spatial scales are considered to understand detailed physical processes and policy implications. These scales are the State level (California), and the Local level (Merced River Basin west to the adjacent Grasslands Ecological Area). At present, there is no other research group that brings together these core components.

\section{Accomplishments}

Our accomplishments include generation of a 100-year baseline climate and a perturbed climate with an imposed 25-year drought for testing the groundwater recovery rates at the Merced BasinGrasslands Ecological Area, advancement of the LBNL Regional Climate System Model to include a state-of-art land-surface/shallow subsurface model (CLM3) with advanced snow and hydrologic processes, development of a TOUGH 2 groundwater module with confined/unconfined aquifer and saturated/unsaturated conditions, with initial CLM3 coupling, modification of the USBR Integrated Groundwater Surface water Model V.2 for the Merced River Basin and the west-side Grasslands regions. In collaboration with Montgomery Watson-Harza, we have also assembled a groundwater Data Management System, performed geophysical logging at Grassland Ecological Area and deployed a Cone Penetrometer Transducer to work in parallel with the LBNL field testing.

Additionally, we have collected baseline water supply and use data for agricultural and urban districts in the Central Valley and other regions of California, formulated an electricity groundwater-pumping model, calibrated the model using regional water-source data, and obtained preliminary estimates of historical groundwater pumping for selected regions in the Central Valley. 


\title{
Next-Generation Codes for Modeling Subsurface Processes
}

\author{
PIs: George Moridis, Stefan Finsterle, and Eric Sonnenthal
}

\section{Project Description}

The objective of this work is the development of a new generation of codes to extend, couple, streamline, and unify the processes and capabilities currently implemented in the TOUGH family of multiphase flow. The increased complexity of environmental and energy resources problems indicates a need to refine hydrodynamic property descriptions and to incorporate new, relevant processes that affect the fate of fluids and contaminants. Moreover, the code should be designed for easy incorporation of geophysical modeling capabilities to enable forward and inverse modeling studies designed for characterizing heterogeneity and for reducing model structure errors.

In order to achieve these scientific goals and to preserve world-wide acceptance of TOUGH in geothermal, environmental, hydrocarbon resource recovery, radionuclide transport, and $\mathrm{CO}_{2}$ sequestration applications, the next generation of codes for modeling coupled subsurface processes must be based on a solid, transparent, and uniform computing platform with a flexible data structure, modular architecture, and coherent implementation of features based on established coding conventions and well-defined interfaces. The objective of re-engineering TOUGH is to ensure a framework within which the new capabilities can be developed and maintained in a safer, more efficient, traceable, and transparent manner.

\section{Accomplishments}

In FY2004, the functional requirements of the new-generation code (called temporarily TOUGHFx) were determined. These incorporate all the requirements reflected in the capabilities currently available in the TOUGH2 family of codes, and include the new set of capabilities needed to address the next generation of problems in subsurface science. FORTRAN95 (and/or FORTRAN 2000/2003/J3, the new upwardly-compatible extension of the current FORTRAN95 standard) was selected as the language for the TOUGH-Fx code. A set of strict standards governing the present and future coding of TOUGH-Fx was developed and adopted. Significant progress has been made in the coding of TOUGH-Fx. Thus, objects and classes were defined for all parameters, properties, and conditions. All routines describing (a) hydraulic properties and their changes, (b) water properties covering spectrum from the compressed subcooled ice to superheated steam, (c) air properties, (d) real gas properties and gas solubility in water, and (d) VOC properties were entirely rewritten according to the aforementioned standards, and were placed in new modules with complete data encapsulation. New routines were written for data input and output (using the current input structure and maintaining backward compatibility), time advancement, and solution convergence. The first version of a working main "core" of the TOUGH-Fx code employing universal dynamic memory allocation was completed and tested. 


\section{Advancing the Next Generation of Rock-Fluid Imaging and Stimulation Technologies}

Principal Investigators: Garrison Sposito, Kurt Nihei, Steve Pride, Ernest Majer

\section{Project Description}

Increasing production in oil recovery operations and enhancing removal of contaminants from groundwater aquifers are major goals in petroleum and environmental engineering, respectively. Seismic wave stimulation is emerging as an important technology for improving efficiency in achieving these goals because it is both economical and ecologically clean, and it overcomes limitations of existing enhanced recovery methods. A central issue in developing seismic wave stimulation as a mature, reliable field technique, however, is determining the fundamental physical mechanisms by which applied seismic waves mobilize pore fluids.

In this project, we are developing a systematic, comprehensive continuum-mixtures approach based on partial differential equations in order to identify the mechanisms responsible for the stimulation effect and to assess its magnitude numerically. The major goal is to provide a solid theoretical basis in continuum physics to advance the next generation of subsurface imaging methods and fluid stimulation technologies used for hydrocarbon recovery and groundwater remediation.

\section{Accomplishments}

During the first year of the project, we derived partial differential equations based on a generalization of the Biot model of poroelasticity to quantify the impact of seismic wave excitation on a homogeneous fully-saturated porous medium. As an illustration of the model, we formulated a boundary value problem corresponding to recent seismic stimulation laboratory experiments with sand cores. An analytical solution then was derived accounting for spatial and temporal variations in total dilatational stress and pore pressure in response to low-frequency stress pulsing. It was shown that fluid phase pressure fluctuations can be related to porosity changes and that the precise dependence of these changes on the pore pressure pulsing frequency can be established. The analytical solution was modeled numerically with elasticity and hydraulic data for experimental soil samples and with stimulation characteristics. The results of our study showed that a maximal porosity increase can be achieved by pulsing in the frequency range of $20-100 \mathrm{~Hz}$, which is in good agreement with that used in seismic wave stimulation. We demonstrated that the contribution of the motional mode in which the solid and fluid phases undergo out-of-phase displacements is more significant than that of the mode in which the displacements of the solid and the fluid are in phase. Sensitivity analyses also indicated that the peak frequency to induce a maximal relative porosity change is proportional to initial permeability, Young's modulus, and the Poisson ratio for the porous medium.

We also developed coupled differential equations of mass and momentum balance to describe dilatational wave propagation and attenuation in elastic porous media bearing two immiscible viscous fluids, including the effects of inertial coupling. Our momentum balance equations generalize all earlier work on this problem and reduce to the well-known Biot model of poroelasticity when specialized to a porous medium containing a single fluid and an elastic solid. After Fourier time-transformation, these equations can be decoupled into three Helmholtz equations that have complex-valued dependent variables and complex-valued, frequencydependent eigenvalues. In the next phase of this project, we shall derive a time-domain representation of the Helmholtz equations and use the resulting partial differential equations to model numerically the dynamic response of an unsaturated porous medium to low-frequency excitation characteristic of seismic stimulation. 


\section{Project Description}

The purpose of this project is two-fold: 1) To understand the nature, structure, and reactivity of several important naturally occurring nanoparticles, and 2) to build a program for the continued such investigation, wedding the efforts of several divisions at LBNL and several UC Berkeley faculty research groups. Specifically, well-characterized mineral nanoparticles (oxyhydroxide and sulfide) will be used to study aggregation and growth processes in the presence of sorbed organic species. This work will feature studies to determine the geometry of specific organic species bound to the particle surfaces. Other experiments will probe the initial nucleation behavior and morphology of mineral nanoparticles in the presence of either watersoluble or water-insoluble organics. The research will extend the methodology learned using large single-crystal surfaces to nanoparticles consisting essentially only of surface, and will include development of techniques to extract nanoparticle size distributions in situ during aqueous formation processes. The methods employed will utilize x-ray scattering (both diffuse and wide-angle) and spectroscopy (EXAFS, NEXAFS) performed at the ALS, SSRL and the APS, and real-time small angle scattering studies (SAXS) at SSRL. The results of this work will serve as a base for detailed studies of other natural nanoparticles, as well as give insight into environmental reactions of anthropogenically-produced nanoparticles.

\section{Accomplishments}

$\mathrm{ZnS}$, a tetrahedrally coordinated semiconductor and also a naturally occurring nanoparticulate, is a good model system because the small free energy difference between the cubic (sphalerite) and hexagonal (wurtzite) phases permits investigations of phase stability at small particle size. When $\sim 3 \mathrm{~nm} \mathrm{ZnS} \mathrm{nanoparticles} \mathrm{are} \mathrm{heated} \mathrm{in} \mathrm{vacuum}\left(350\right.$ to $\left.750{ }^{\circ} \mathrm{C}\right)$, sphalerite transforms to wurtzite, implying reversal in phase stability in small particles. Coarsening of $3 \mathrm{~nm} \mathrm{ZnS}$ nanoparticles in a hydrothermal environment yields wurtzite only on the surface of primary sphalerite particles. Crystal growth of wurtzite stops when the diameter of the sphalerite-wurtzite interface reaches $20 \mathrm{~nm}$, suggesting a dependence of the transformation mechanism on particle size. In subsequent work a mechanistic explanation for this phenomenon was developed (Huang et al. in press). In 2003 we reported the water-driven structural transformation in $\mathrm{ZnS}$. Research in 2004 tackled the kinetics of this reaction using both wideangle x-ray diffraction to follow the structural state, and small-angle x-ray scattering to monitor aggregation. The experiments explored the dependence upon both water concentration and temperature, and will soon be submitted (Goodell et al. in prep.). Coarsening: ZnS nanoparticles in water with no organic capping $\left(\mathrm{H}_{2} \mathrm{O}-\mathrm{ZnS}\right)$ and mercaptoethanol [ME]-capped $\mathrm{ZnS}$ were coarsened in a hydrothermal environment. Early crystal growth of both $\mathrm{H}_{2} \mathrm{O}-\mathrm{ZnS}$ and MEcapped $\mathrm{ZnS}$ was found to occur predominantly via crystallographically-specific oriented attachment (OA). Twins and stacking faults formed in the coarsened ME-capped $\mathrm{ZnS}$ whereas more complex, closely spaced twins, stacking faults, and intergrowths occurred in coarsened $\mathrm{H}_{2} \mathrm{O}-\mathrm{ZnS}$. Eventually, diffusion-controlled growth removes surface irregularities arising from OA yielding rounded particles with complex internal structures (Huang et al. in review). $\alpha-\mathrm{FeOOH}$ nanoparticles: $\alpha-\mathrm{FeOOH}$ (goethite) is present in most soils and marine sediments. Of particular interest is the rate of goethite surface reactions with natural or anthropogenic toxic sorbates and organic soil components and how these are affected by particle size. By a combination of heat treatments and aging, monodispersed nanogoethite has been synthesized and characterized with DLS (dynamic light scattering), SAXS (small angle x-ray scattering), EXAFS (extended x-ray absorption fine structure) spectroscopy, and TEM imaging. Real time SAXS studies of growth suggest that $5 \mathrm{~nm}$ primary goethite particles grow by OA, while larger particles coarsen mainly through a diffusive process. EXAFS analysis of sorption of $\mathrm{Hg}, \mathrm{Zn}, \mathrm{AsO}_{4}$ and $\mathrm{Cu}$ suggest that the number and type of reactive surface sites change with crystallite habit and diameter (Waychunas et al., in review; Kim et al., in prep.). Using the PDF method with high energy x-rays $(105 \mathrm{KeV})$ we are calculating high resolution pair correlation functions for goethite particles in the $4-75 \mathrm{~nm}$ range. Additionally, we have shown that the surface coordination of $\mathrm{O}$ by $\mathrm{Fe}$ has a strong effect on the $\mathrm{O}$ K-edge spectra, and can be used to characterize the proportions of different types of $\mathrm{O}$ sites on the nanoparticle surfaces. 


\section{Environmental Energy Technologies Division}

LB02028

Miniaturized System for Particle Exposure Assessment

Principal Investigator(s): Michael G. Apte, Lara A. Gundel and Anthony D. Hansen

\section{Project Description}

Human exposure to anthropogenic airborne particulate matter (PM) is a high priority environmental health research issue. The purpose of this project has been to prove the concept and develop a prototype of a new generation PM of sensors. These sensors are called the Miniaturized System for Particle Exposure Assessment (MSPEA). They are designed to address a major problem faced by environmental, exposure assessment, and health research communities: current PM monitoring devices for use in studying population exposures to airborne PM are large, expensive, noisy and intrusive. Thus they are inappropriate for use where study of individual PM exposure measurements of hundreds or thousands of people must be conducted. The MSPEA devices are intended to integrate measurement of PM mass concentrations and apportionment of the sources of the major constituents of the PM simultaneously, in real time. Surprisingly, current PM instrumentation for exposure assessment relies on technologies developed in the late $19^{\text {th }}$ Century. This project uses microelectronic technologies developed over the last two decades that lend themselves to miniaturization, high sensitivity, and low cost production. The LDRD funding enabled us to develop and test a proof of concept prototype MSPEA and fabricate (but not test) a second-generation MSPEA sensor.

We use thermophoresis, a particle collection mechanism effective for particles in the size range of nanometers to about 10 microns. We have developed a low power and compact PM collection system that does not require a large pressure drop for fine particle collection. In the MSPEA, particles are collected onto a quartz resonator sensitive to mass changes $<1 \mathrm{ng}$. The optical properties of the most abundant and harmful airborne particle species (i.e., diesel exhaust, environmental tobacco smoke (ETS), and woodsmoke) are well understood, enabling us to design simple optical probes that can differentiate between them when they are collected on a reflective surface. Advances in microelectronic light sources and detectors enable us to integrate miniature optical probes into the miniature particle mass monitor. The physics of these methods scale well together and can be integrated into microchip-sized devices. At the MSPEA sample inlet, a gravitational particle size separator is used to reject particles $>2.5$ micrometers.

Accomplishments

During the preceding two years (FY02 and FY03) we demonstrated PM collection onto quartz crystal resonators as a sensitive and stable method for particle mass detection in real time. We also successfully demonstrated that a simple UV/NIR optical reflectance probe could be used to track particle loading on the reflective resonator substrate in real time, and that materials with enhanced UV absorbance such as ETS could be distinguished from black body absorbers such as diesel soot on this collection surface.

During FY04 we used the small additional allotment of LDRD funding to fabricate, but not test, a second generation MSPEA prototype. This device will be tested during future experiments with funding from a new research grant stimulated by this LDRD project. Progress made during the three years of this LDRD provided the proof of concept and preliminary results necessary to secure follow on support in two new projects. 
Determining the Light-Absorbing Properties of Aerosol Particles Principal Investigator: Thomas Kirchstetter

\section{Project Description}

The purpose of this project is to develop methods to determine light absorbing properties of climatically relevant atmospheric aerosol particles. Recent studies suggest that light absorption by aerosols is the second largest component of direct climate forcing after carbon dioxide, and impacts the hydrological cycle and photochemical air pollutant formation. Presently, limitations in analytical methods prohibit accurate determination of absorbing properties of aerosols and thus their realistic representation in air quality and climate models. To date, no method measures light absorption by the organic carbon (OC) fraction of aerosol mass, and measurements of the spectral light absorbance of black carbon (BC) are scarce and inconsistent.

This research will develop light transmission methods to measure the light absorbance by collected aerosol particles over the near ultraviolet to the near infrared spectral regions. The methods will be developed in combination with solvent extraction and thermal analysis methods that separate OC and BC, thereby allowing for light absorption to be apportioned to these different classes of carbonaceous material in aerosol samples.

\section{Accomplishments}

A major accomplishment has been the development of a spectrometer based light transmission method, which has been applied to aerosols produced from the most significant sources globally: transportation (gasoline and diesel) and burning biomass. We observed that light absorption by the motor vehicle aerosols exhibited relatively weak wavelength dependence, whereas light absorption by the biomass smoke aerosols had much stronger wavelength dependence. The stronger spectral dependence was the result of enhanced light absorption at wavelengths shorter than $600 \mathrm{~nm}$, which was attributed to OC in the aerosol particles. We deduced that OC in addition to BC in biomass smoke absorbs solar radiation. Absorption efficiencies and imaginary refractive indices were determined for the OC extracted from biomass burning samples and the BC in motor vehicle dominated aerosol samples. Collaboration with scientists at NASA has led to incorporation of these results in global climate models.

Another significant accomplishment has been the development of a thermaloptical analysis method that simultaneously monitors light absorption by an aerosol sample while it is heated to temperatures of evaporation, decomposition and combustion. This method was first applied to the simple case of an aerosol composed entirely of lightabsorbing BC, which demonstrated that this method accurately determines the mass of $\mathrm{BC}$ in an aerosol sample and its absorbance as a function of wavelength over the near ultraviolet to the near infrared spectral regions. This method has also been applied to measure spectral absorbance and BC mass in aerosol samples collected in a roadway tunnel carrying gasoline and diesel vehicles.

Continuing work involves application of the thermal-optical method to biomass smoke aerosols, and an investigation of the applicability of the aethalometer, a widely used light transmission method, to monitor atmospheric BC concentrations. 
Novel High-temperature Membranes and Electrocatalyst Structures for Fuel Cells Principal Investigator(s): Nitash Balsara, Kyungoul Baek, Robert Kostecki

\section{Project Description}

Polymer-electrolyte (PE) fuel cells are in a dramatic period of rapid development, but there remain serious problems that need to be solved before they can achieve significant market penetration. These problems include significant efficiency losses due to the poor oxygen-electrode catalyst, prohibitive cost of current materials, and the need to find a suitable high-temperature membrane structure. The purpose of this project is to find new materials that address the above problems, and understand better the underlying fundamental processes. The two main goals of the project are: 1) develop new high-temperature proton-conducting membranes, and 2) study electrocatalysis and develop new catalyst structures to lower efficiency losses.

\section{Accomplishments}

To accomplish the first goal, we were to synthesize and characterize durable nanostructured polymer membranes that have high proton conductivity at $120^{\circ} \mathrm{C}$ while maintaining low gas permeability. The morphology of the membrane consists of an ion-conducting network in a nonconducting matrix. For this, we synthesized two types of block copolymers with narrow molecular weight distributions, where non-conducting segment is to provide mechanical strength and control permeability and conducting segment is to provide ion (proton) transportation. A series of welldefined (P $\alpha$ MeSt-PIB-P $\alpha$ MeSt) copolymers with different volume fraction of $\alpha \mathrm{MeSt}(0.2 \sim 0.6)$ were synthesized by living cationic polymerization, followed by the sulfonation of $\alpha \mathrm{MeSt}$ segments with sulfur trioxide to obtain sP $\alpha$ MeSt-PIB-sP $\alpha M e S t)$ copolymers. The second type of polymer that we have synthesized is a diblock copolymer of cinnamoylethyl acrylate (CEA) and protected neopentyl styrenesufonate (NSS). The CEA block will serve as the matrix and will be crosslinked by UV radiation after the membranes are made.

To accomplish the second goal, our primary task is to develop a new diagnostic technique(s) to provide valuable information for designing nano-structured catalyst electrodes for the proton exchange polymer (PEM) fuel cells. We intend to improve the platinum-based electrocatalyst performance by investigating in detail the particle-size and particle-shape effects, i.e., correlate catalyst morphology with electroreactivity. The dimensions of a nano-probe suitable for detection and characterization of electrochemical processes at the nanometer scale resolution must be equal or smaller than surface morphology features at the electrode/electrolyte interface. In our initial attempts to micro-fabricate such an electrochemical AFM nano-probe we used a substantially modified platinum STM tip and a probe microfabricated from a thin Pt wire . However, imaging at nanometer-scale resolution failed to detect small surface features and revealed that the new nano-probes were highly unstable, mainly due to their low resonance frequency. They deteriorated quickly because of the tip's poor mechanical properties and tendency to wear out during scanning in AFM contact mode. A new strategy has been developed to overcome the problems that were encountered in our preliminary experiments. This time, commercial ultra-sharp silicon AFM tips with a custom made Pt coating were used. A new coating process was developed to insulate the nano-probes with thin non-conductive films, except for the tip's very end. The parameters of the nano-probe manufacturing procedure were optimized during a meticulous try-and-error experimental work. Further development of the nano-probe will continue in the second year. 


\section{Nanostructured Cathodes for Efficient Organic LED}

Principal Investigators: Samuel S. Mao and Richard E. Russo

\section{Project Description}

The purpose of this project is to develop structured cathodes for achieving high efficiency organic light-emitting diode (OLED) devices. Since the remarkable discovery of electroluminescence from organic molecules and conjugated polymers, there has been an increasing interest in the development of OLEDs for lighting and display applications. However, a major limitation for OLED technology is inefficient injection of electrons from cathode to the organic electroluminescent layer.

A basic OLED structure consists of a thin layer of organic electroluminescent medium sandwiched between a cathode and an anode. With electrons injected from the cathode into the organic material's lowest unoccupied molecular orbital (LUMO) and holes from the anode into the highest occupied molecular orbital (HOMO), the device emits light upon the application of a voltage greater than a threshold bias. In principle, the cathode work function should be small enough to match the LUMO of the organic material, so that it is efficient to inject a steady supply of electron for light generation. While alkali metals have the lowest work function of all elemental metals, their highly reactive nature has been a major barrier for the realization of long lifetime, high efficiency OLED devices. It is therefore the objective of this research to look for alternative stable electrode materials to improve the efficiency of OLED devices.

\section{Accomplishments}

To overcome the barrier of inefficient electron injection in OLEDs, we developed a concept of utilizing stable nanoscale materials as the OLED cathode layer. In the past year, we established a nanomaterials fabrication system and performed experiments by fabricating OLED cathode layers using carbon nanotubes as well as low work function nanoclusters (e.g., $\mathrm{CeB}_{6}$ ). Comparison experiments with standard Al film cathodes clearly demonstrated that the turn-on voltage of OLED devices could be reduced by using carbon nanotubes as the cathode layer (Figure 1). To further improve the efficiency of electron transport at the interface between carbon nanotubes and the organic medium, we performed additional experiments to modify the wetting property of the electroluminescent polymer (polyfluorene). Applying modified polyfluorene mixed with carbon nanotubes, the luminescent efficiency of OLED devices was found to be significantly increased.

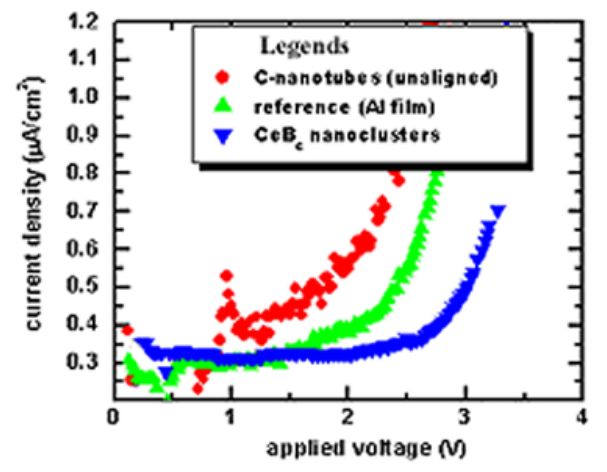

Figure 1. Current-voltage relation for OLED devices using three different types of cathodes. Note that, because of their relatively low conductivity, cathodes built with low work function nanoclusters $\left(\mathrm{CeB}_{6}\right)$ on $\mathrm{Al}$ films did not show improved characteristics. 
Evaluating aerosol effects on regional and global energy and water budgets Principal Investigator: Surabi Menon

\section{Project Description}

A large component of the uncertainty in current climate projections/predictions are related to aerosol climate effects. Both aerosols (e.g. sulfates, organic and black carbon) and greenhouse gases play a strong role in modulating the energy and water cycles. While climate effects from greenhouse gases are relatively well quantified, aerosol climate effects through the direct, semidirect, thermodynamical and indirect processes cannot be quantified without a large uncertainty due to complexities in evaluating these various processes. The work proposed through the LDRD project is focused on evaluating the role of absorbing aerosols on the energy and water cycles and advancing current understanding of the aerosol indirect effect in relation to other climate forcings via a series of climate simulations.

We use the NASA Goddard Institute for Space Studies (GISS) coupled aerosol-climate model, along with satellite products of relevant aerosol/cloud fields, to assess the two issues listed above. Several climate simulations are being carried out to understand: (1) effect of vertical distributions of absorbing particles on the energy and water budgets; (2) treatment of precipitation processes in the model and its response to differing aerosol concentrations in convective and stratiform clouds. Understanding the response of cloud systems and the changes in water and energy budgets due to aerosols and aerosol-cloud interactions, and constraining model simulation of climate products with satellite data, will help narrow current uncertainities in model predictions. Ongoing work related to climate changes for 1950 to 2050 due to aerosolcloud interactions will provide an estimate on the expected trends and magnitude of changes in the energy/water budgets due to aerosols.

\section{Accomplishments}

The effects of aerosols on climate during the Chinese summer monsoon were studied, based on simulations with the GISS climate model. We found greater changes in simulated temperature, radiation and precipitation for aerosols confined below $\sim 550 \mathrm{hPa}$ (above $\sim 3700 \mathrm{~m}$ ), which suggest that climate effects of aerosols (especially absorbing aerosols) are stronger when they are located at higher levels in the atmosphere. In addition we also analyzed regional precipitation changes over China and India due to differing amounts of black carbon from fossil/bio-fuel sources. In both regions, precipitation changes appear to correspond to the amount of atmospheric heating, with stronger radiative fluxes (heating) in the atmospheric column corresponding to larger changes (positive) in precipitation though these may also partly be dependent on surface/meteorological conditions.

Regional changes in climate diagnostics were evaluated for aerosol effects on stratiform and convective clouds. Including aerosol-convective cloud effects substantially lowers the indirect effect estimates (since longwave effects are comparable to shortwave effects), and level of precipitation initiation is shifter to higher atmospheric levels. Precipitation appeared to decrease significantly in the Amazonia when accounting for increased cloud droplets in biomass burning regions in warm convective clouds.

Preliminary work is being carried out to detect aerosol-cloud effects from analysis of satellite data. Case studies were compiled to understand the relationship between aerosols and cloud properties at different locations across the globe and these will be compared with model climate simulations for all cloud types to help constrain model predictions of the indirect effect. 


\section{Long-term Global Energy Demand and Carbon Emissions Scenarios}

Principal Investigators: Jayant Sathaye, Mark D. Levine, Lynn Price, Stephane de la Rue du Can, Jonathan Sinton, Ernst Worrell, and Nan Zhou

Project Description:

The Intergovernmental Panel on Climate Change (IPCC) has developed a set of baseline global scenarios that report on the anthropogenic greenhouse gas emissions from all sources up to the year 2100 by four geographic regions. The scenarios were developed using a set of models that lacked a detailed representation of demand-side drivers and technologies. These baseline scenarios provide the overall conceptual framework in which emissions of greenhouse gases and their impacts are analyzed.

The purpose of this LDRD project is to develop scenarios of energy demand by end-use for ten global regions consistent with one or more IPCC scenarios. Surprisingly, the global greenhouse gas and energy demand scenarios developed by IPCC, other intergovernmental organizations (e.g., UN, International Energy Agency), international oil companies, and national governments are lacking in end-use detail. Most of the scenarios provide no more detail than total energy use by buildings, industry, and transport by region, energy type, and year. Few, if any, of the underlying drivers (energy service demand, technology type, usage, saturation, efficiency) are included in the scenarios. As a result, it is virtually impossible to understand or interpret the scenarios in terms of current or future energy demand at the level of detail required by both policymakers and end-use energy analysts. The LDRD project will for the first time provide such information to the community that analyzes climate change.

Accomplishments:

Through our participation in the IPCC Working Group III, we are collaborating with the industrial, transportation and building sector teams as well as the long-term emissions scenarios team. We are working closely with them in the formulation of sectoral models and in developing an end-use energy consumption data base for each sector. In addition, the Asia Pacific Energy Research Centre (APERC) based in Japan will work with LBNL in procuring demand-side data and information from their 22 member countries.

With respect to the disaggregation of the IPCC scenarios, we have initiated work on the buildings sector in China and the US steel sector. Disaggregation of the IPCC's Scenario B2 has been completed for the China buildings sector, and will be reported at an upcoming IPCC meeting in January 2005. Sample results are shown in the figure below.

China (B2 Marker Scenario)

Driver variables for bottom-up characterization of buildings sector Buildings Sector, Primary Energy -

Asia and China

Primary Energy - China
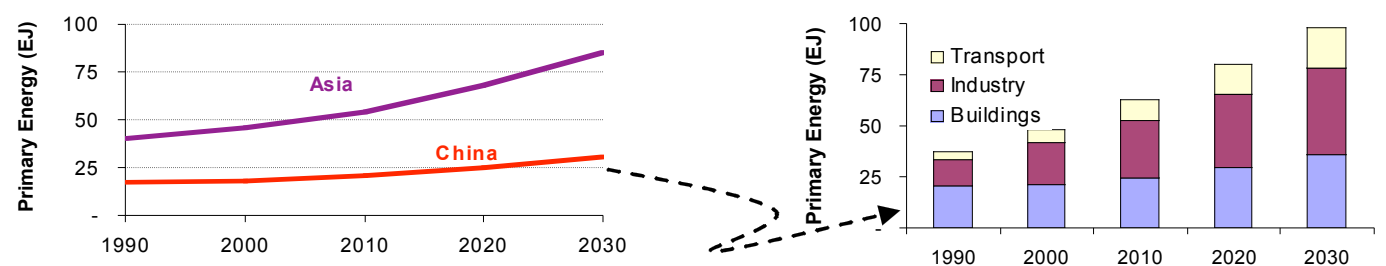

\begin{tabular}{lccc} 
Driver Variables & $\mathbf{2 0 0 0}$ & $\mathbf{2 0 3 0}$ & AAGR \\
\hline GDP (trillion yuan) & 9.1 & 58.7 & $6.4 \%$ \\
Population (millions) & 1,268 & 1,451 & $0.5 \%$ \\
Share urban population & $33 \%$ & $61 \%$ & $2.1 \%$ \\
Commercial building area (billion m2) & 8.7 & 36.9 & $4.9 \%$ \\
Per capita living space--urban (m2/person) & 19.9 & 36.7 & $2.1 \%$ \\
Per capita living space--rural (m2/person) & 24.9 & 39.0 & $-0.1 \%$ \\
Household size--urban (persons) & 3.2 & 3.1 & $-0.4 \%$ \\
Household size--rural (persons) & 4.4 & 3.9 & $1.5 \%$ \\
\hline Building Energy Demand (EJ) & 21.2 & 35.8 &
\end{tabular}


Evaluation of Dynamic Air Quality Impacts of Distributed Generation

Principle Investigator(s): Robert Van Buskirk \& Shaheen Tonse

\section{Project Description}

A critical challenge for the atmospheric sciences is to understand the anthropogenic impacts on atmospheric chemistry over spatial scales ranging from the urban to the regional, and ultimately to the global, and over corresponding time scales ranging from minutes to weeks and ultimately annual trends. A similar challenge for energy policymakers is to integrate an understanding of air quality impact dynamics into the economic dynamics of energy supply and demand. The need for dynamic analysis of emissions impacts from the energy sector have substantially increased with a new focus on emerging distributed energy resources (DER). We are building a long-term capability to perform integrated analysis of the dynamic economic, meteorological and air chemistry impacts and effects from distributed generation (and ultimately from a diversity of combustion-based emissions.) Our approach explicitly integrates the economic demand/supply price dynamics with meteorology, emissions and atmospheric chemistry dynamics for application to optimized emissions regulation. The problem is challenging due to the inherent non-linearity of the photochemistry at all spatial scales, the difficulties in simultaneously representing atmospheric fluid dynamics at different scales within a numerical model, and the complex geographic and temporal dynamics of the economics of electricity markets and distributed energy demand and supply. Our LDRD project has initiated this effort by building a modeling system that integrates component models that are the product of previous research.

This LDRD effort is a first step in building an integrated dynamic modeling capability to estimate the incremental air quality impacts of DER emissions using integrated economic, meteorological and atmospheric chemistry models in order to provide estimates of time-dependent impacts from DER emissions in central California.

\section{Accomplishments}

Our core accomplishment for this study has been to successfully implement an integrated simulation of the Central California Ozone Study (CCOS) episode of 30 July to 2 August 2000 and examine a variety of incremental DER emissions sensitivity scenarios. A key unanswered question of DER emissions and ozone production is what will the impacts of moving emissions closer to population centers as energy resources are moved closer to the attendant energy demand.

For the simulated CCOS episode of Central California, the incremental impact on Ozone for a shift to emissions that are more highly correlated with population distributions is to move emissions associated with electricity generation "upwind." A common low-level meteorological flow pattern during warm summer days consists of a sea breeze channeled through the San Francisco Bay Area (SFBA) as cool air over the coastal waters flows through a gap in the coastal mountain range at the Carquinez Straight.

Incremental emissions for both diesel-powered and gas-powered generation scenarios were simulated where electricity generated by central plants was assumed to be shifted to DER's correlated with population density. From the perspective of high density population centers, both cases show nearfield reduction of ozone by NOx titration, and an increase in ozone farther downwind. The net impact is an incremental decrease in peak daily ozone during this episode and a smaller, but more widespread increase over the less populated areas of the Central Valley.

We are in the process of refining the detail and generality of our characterization of the spaciotemporal characteristics of incremental DER ozone impacts for a variety of characteristic DER emissions scenarios. 


\section{Genomics Division}

LB04026

Making the Most of Sequencing: Improved Assembly and Comparative Annotation

Principal Investigator: Eugene Myers

\section{Project Description}

Our goal is to efficiently sequence and annotate genomes using a comparative approach. We are building a whole genome assembler that will deliver $99-99.5 \%$ of a genome as opposed to the $97 \%$ typically returned by today's software. A highly sensitive, yet efficient whole genome aligner is being built that will find the most-likely evolutionary relationships between genome segments and will further correctly align sequences at the base-pair level. Finally, a symmetric comparative gene-finder that simultaneously finds the best annotation of all orthologs in the input genomes is underway. A similar RNA-gene finder and cis-regulatory element finder are also potential objectives. Each component feeds the next: more efficient assembly permits more sequences, each more complete as input to the comparative step, and better whole genome alignments are critical to more robust comparative gene finding.

A better assembler requires better repeat resolution and we will pursue a number of algorithmic strategies that attempt to exploit micro-heterogenieties between repeats and the integrity of paired reads. But before separating repeats, one must first be aware of them and their structure. We develop a "string graph" concept where repeats are collapsed but clearly visible. Our whole genome comparator is based on (1) novel methods for rapidly finding local alignments, (2) a maximum-likelihood based approach to genome rearrangements, and (3) sophisticated gap-scoring schemes to ensure that the base-level details of an alignment are correct. Finally, the best possible use of comparative data is to build the best possible 1dimensional gene finders (i.e. HMMs) and then constrain them to predict the same orthologous protein in all $\mathrm{k}$ species simultaneously. While computational intractable as stated, we are taking advantage of sparsity to achieve the goal without sacrificing rigor.

\section{Accomplishments}

On the topic of assembly we have developed an overlapper, multi-aligner, and read corrector to date. The center piece is the component that makes decisions about how to put the pieces together. We have develop a novel transitive removal algorithm that makes the construction of a string graph very efficient. We have further developed smoothers and network flow based algorithms that analyze this string graph so as to determine the repeats and the tours through the graph that could spell the underlying target sequence. We expect to have a complete end-to-end system by June of 2005. In a related work we have developed a syntenic assembler and produced several assemblies of the $D$. simulans genome against the background of $D$. melanogaster in collaboration with Chuck Langley at UC Davis.

We have developed a whole genome local alignment finder that very efficiently and sensitively finds local alignments between two genomes. We are currently developing tools that identify repetitive structures in each genome and between the genomes as a preliminary step in finding the maximum likelihood correspondence between two genomes and towards a model of alignment that attempts to determine a likely ancestral sequence.

For gene finding, we have developed the HMM infrastructure needed for our approach, and developed a method for identifying regions that are mostly likely to be coding based on their evolutionary relationship to other genomes. This will allow us to restrict the space of alignments that the HMMs need to explore. 
Identification and Characterization of Conserved Noncoding Sequences using

Comparative Genomics and Transgenic Technology

Project Description

Principal Investigator: Eddy Rubin

Gene regulatory sequence embedded within the $98 \%$ of the human genome is noncoding. Despite the importance of these sequences in gene regulation, our ability to identify and predict functions for noncoding DNA is extremely limited due to the difficulty in distinguishing them from other noncoding sequences. The development of computational tools for comparative genomic analysis has provided a strategy to screen for evolutionarily conserved sequences but this approach does not link the sequences to function or reveal the temporal and spatial patterns of gene regulation. Traditionally, generation of transgenic mouse lines has been used to identify and functionally characterize non coding sequences. However, this has been a slow, labor intensive and expensive process. Recently, we have explored the generation of transient transgenic mice as a feasible in vivo high throughput system for the characterization of conserved sequences impacting gene expression in mouse embryos (1). Briefly, highly conserved noncoding sequences fused to a minimal promoter and linked to B-galactosidase were used to generate transgenic embryos that were examined for spatial and temporal patterns of gene expression between embryonic day 10-13. On its own, this vector is unable to drive the expression of B-galactosidase gene but when fused to the test sequence with gene enhancer activity, spatial and temporal patterns of expression can be determined reproducibly in a relatively short period of time.

Accomplishments

One of our most significant accomplishments using comparative genomics and transient transgenesis has been the identification of long range enhancers in gene poor regions or deserts (2). Of the nine elements tested, seven elements were shown to be gene enhancers for human DACH. One of these ancient conserved enhancers, Dc2, which displays a human-Fugu identity of $84 \%$ over $424 \mathrm{bp}$ was further characterized. We demonstrated that this conserved element is necessary and sufficient for the enhancer activity of Dc2. In addition to this large overall conservation, we found that Dc2 is characterized by the presence of a large block of sequence (144bp) that is completely identical between human and Fugu. Further in vivo analyses revealed that this 144bp sequence is necessary, but not sufficient, for Dc2 enhancer function. (3)

We have systematically characterized 70 of the 88 highly conserved human-fugu noncoding elements on human chromosome 16 for regulatory activity. Our data show that a large proportion (44/70) of elements tested act as gene enhancers in vivo. The results of this study are available in a public database that we have established to visualize the expression patterns and the localization of genome-wide human-fugu conserved elements (Enhancer Browser for chromosome 16: http://chr16.lbl.gov/). Taken together, these studies support the power of comparative genomics and transient transgenesis to identify and characterize gene regulatory elements scattered throughout the human genome and provide insights into regulation of gene expression. 


\section{Life Sciences Division}

LB04010

Molecular Electron Microscope Tomography: 3D Visualization, Localization and

Quantitative Analysis of Molecular Machines in Cells and Tissues at Molecular Resolution.

Principle Investigator: Manfred Auer

\section{Project Description}

The purpose of this project is to employ electron microscopy tomography in combination with novel genetic tagging and biochemical labeling methods to study the three-dimensional localization and molecular three-dimensional structure of macromolecular machines in their native cellular environment. The goal is to identify the constituting protein components and to determine the architectural building principle of cellular machines in order to understand how these multi-protein complexes achieve their remarkable ability to sense mechanical stress and to transduce this mechanical signal across biological membranes.

Moreover, information about the three-dimensional localization of cellular machines in whole cells and tissue will lead to the development of more realistic mathematical models of higher level, integrative function in cells. This approach will be of particular interest for the study of bacteria in microbial communities (biofilms) that are crucial in bioremediation and biodegradation. Molecular Electron Microscope Tomography is therefore highly relevant to the mission of the Department of Energy and its GTL initiative.

We will continue to develop methods for 1) the 3D visualization and quantitative analysis of tomographic data sets, and for 2) labeling of genetically encoded tags followed by the 3D localization of candidate proteins.

\section{Accomplishments}

We have examined the structure of the mechanoelectric transduction and adaptation machinery of the inner ear hair cells, which is unsurpassed in its elegant and efficient design, and which allows the faithful transduction of mechanical signals such as sound, vibration or gravity into an electrical response that can be interpreted by the brain. We were able to visualize the complexity of this molecular machine and to propose candidate proteins that constitute this multi-protein complex.

We have further extended our approach on the kidney blood ultrafiltration barrier and have started to identify its molecular architecture. The three-dimensional structure reveals a surprisingly complex design of the slit diaphragm of glomerular podocytes, and we are in the progress of further analyzing this cell-cell adhesion complex that is central to the ultrafiltration of blood, and to the disposal of toxic blood components into the urine.

We have recently initiated a collaborative effort with Dr. Bill Costeron, who was one of the first to recognize the significance of bacterial biofilms and is an expert in the biofilm engineering and analysis. In this collaboration we will study bacterial biofilms using electron microscope tomography and novel tag-based labeling strategies, and will therefore contribute to a better understanding of bacterial behavior in their natural habitats. Their reaction to environmental challenges can be tested at molecular resolution, and the molecular structure of the cellular machines necessary for bioremediation and biodegradation (e.g. of cellulose) can be examined. 


\section{Membrane Protein Cryo-electron Microscopy \\ Principal Investigators: Felicia Betancourt and Robert Glaeser}

Project Description

The immediate goals of this project are two-fold: 1) to use cryo-electron microscopy (cryoEM) partnered with single particle analysis to obtain a low resolution model of cytochrome reductase (bc1), a mitochondrial membrane protein complex whose atomic structure is known; and 2) to determine the structure of a membrane protein of unknown structure to the highest resolution possible using high-throughput data collection and image processing methods. Because the technique of single particle analysis has rarely been applied to membrane proteins, questions arise as to whether the film of protein solution formed on the electron microscope grid in the process of sample preparation will be too thin or too thick if a substantial amount of detergent is required to keep the protein in solution. Detergent-solubilized cytochrome reductase will therefore serve as a proof of principle.

Single particle analysis is a viable alternative to x-ray crystallography when the structure of a large protein or protein complex is sought and the target species is refractory to crystallization. However, in order to routinely achieve resolutions that would permit tracing the polypeptide chain(s), the field must advance significantly. In particular, it will be necessary for hundreds of thousands or even millions of images to be selected from micrographs and aligned in silico. We will apply high-throughput image collection to images of a protein of unknown structure with the intent of pushing the resolution beyond the current practical limit of about $1 \mathrm{~nm}$.

\section{Accomplishments}

Our first accomplishment is the computation of a low resolution (between $30 \AA$ and $40 \AA$ ) model of cytochrome reductase from images recorded on the JEOL 4000 electron microscope. It is very encouraging that we only used 944 individual images rather than the 3,000 we had initially speculated would be required for this resolution. Furthermore, we found that the "top" view

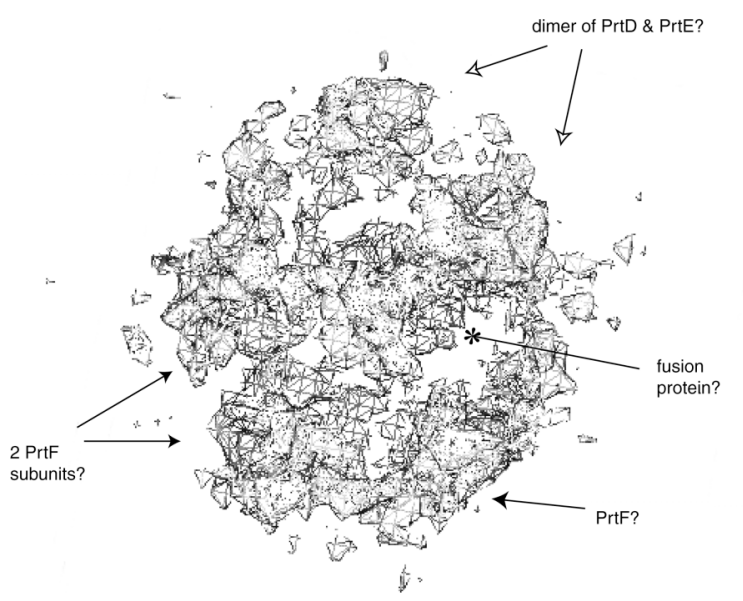

presence of detergent in the protein solution proved to be no obstacle to sample preparation. It is also noteworthy that, at $460 \mathrm{kDa}$, cytochrome reductase is at the low end of the molecular weight range amenable to single particle analysis, so this constitutes yet another challenge we were able to overcome.

Even more exciting, however, is our preliminary model (see left) of the proteasesecreting transporter of Erwinia. chrysanthemi, a Gram-negative bacterium. The transporter is a complex of three gene products (PrtD, PrtE and PrtF), and is believed to span both inner and outer membranes as well as the periplasm in-between. Our model shows a central density that we tentatively identify as the substrate protease. If this is correct, then we have found strong evidence that this transporter unfolds its substrates, a matter of controversy in the literature. 


\title{
A High Throughput Strategy to Identify Protein Complexes
}

\author{
P.I.s Mark D. Biggin, Jian Jin, Ursula Schulze-Gahmen
}

\section{Project Description}

Individual proteins often act as protein complexes that carry out the myriad biochemical tasks within cells. In response to altered environments, these complexes change in their constituent polypeptides and covalent modifications. A major focus of current DOE research directions is to identify and characterize these dynamic molecular machines on a genome wide scale. We propose a novel strategy to purify and identify stable protein complexes with much higher throughput than current methods.

The most common strategy to isolate protein complexes from crude cell extracts uses protein specific affinity tags. Each tagged complex must be purified one-at-a-time from a distinct genetically engineered strain. Then, polypeptides in the complex are identified by mass spectrometry. We propose to develop an alternative strategy to greatly increase the number of protein complexes that can be identified. This novel "tag-less" strategy uses a multidimensional, highly parallel chromatography platform to divide a crude bacterial extract into 10,000-20,000 fractions, then use LC MALDI mass spectrometry to identify the polypeptide constituents in each fraction from a tryptic digest of the whole fraction. This strategy should permit rapid identification of most stable water soluble complexes in an organism from one preparation of cells. During the course of this pilot project, we are performing proof of principle experiments on $E$. coli extracts, generating and analyzing several hundred factions, including some that contain known protein complexes as positive controls. To validate our tag-less strategy, we are comparing the known and putative complexes purified in this way to the same complexes isolated using the standard tagged strategy for affinity purification.

\section{Accomplishments}

To pilot the tagless strategy, we first identified by western blot and purified bacterial RNA polymerase using a three column purification scheme: S400 Sephacryl, mono Q, and hydrophobic interaction. From a $6 \mathrm{~L}$ culture, we obtained $1 \mathrm{n}$ mol of pure protein. We showed that we can efficiently detect all polypeptides in this complex using only 1/10,000 of this preparation of protein using both LC ESI Qtof and LC MALDI tof/tof mass spectrometry methods. RNA polymerase is a relatively abundant protein, present at around 600 molecules per cell. But the fact we can easily detect this implies that with improved sample concentration we should be able to detect the lowest abundance complexes present at one molecule per cell.

The above experiment employed targeted purification of a known protein. To test if the blind screening approach at the heart of the tagless strategy is feasible, we have first simply performed SDS PAGE on a series of factions that in effect search a small part of our proposed multi dimensional parallel chromatography scheme. Every other fraction from the mono Q fractions used to purify RNA polymerase were each subjected to fractionation by hydrophobic interaction chromatography (HIC). Manual inspection of these fractions reveal five sets of co migrating polypeptides that are potential protein complexes, suggesting that indeed it is possible to discover complexes this way. We are now in the process of determining the sequence of these putative protein complexes.

We have also established the affinity tag method to isolate two E. coli complexes: acyl carrier protein (ACP) and RNA polymerase. We correctly identified the know subunits of RNA polymerase and detect a set of polypeptides associated with ACP. 


\title{
Analysis of Complex Phosphorylation Patterns in a Key DNA Repair Protein By Coupling Surface Plasmon Resonance and Tandem Mass Spectrometry
}

\author{
Principal Investigator: Priscilla K. Cooper
}

Project Description

The purpose of this project is to develop an innovative approach that integrates mass spectrometry (MS) and surface plasmon resonance (SPR) to improve sensitivity and efficiency of characterization of complex phosphorylation patterns and mapping of individual phospho-sites. Phosphorylation and dephosphorylation events are the switches that control activities, protein partnerships, and subcellular localization of the molecular machines that respond to DNA damage. Identification and characterization of the phosphorylation status of key DNA repair proteins in response to DNA damage is therefore central to understanding regulation of functions required for maintaining genomic integrity. However, the low cellular abundance and multiple phosphorylations common for these proteins pose a significant technical challenge to mapping their phospho-sites even by mass spectrometry, the most sensitive technique currently available.

We propose to couple two technologies, SPR and tandem MS, to quantitatively optimize enrichment and recovery of phosphopeptides from protein digests to improve sensitivity of detection and ability to map phospho-sites. The strategy will initially be applied to the multifunctional human DNA repair protein XPG, which is involved in three different DNA repair pathways and in their coordination with each other and with other vital cellular processes including transcription. XPG is essential postnatally and when defective results in death in infancy or early childhood.

Accomplishments

We have applied immobilized metal affinity chromatography (IMAC) with an NTA chipbased SPR platform to enrich for a custom-designed model phosphopeptide. We optimized enrichment and recovery strategies that enabled MS identification and sequencing of the phosphopeptide in the presence of at least 10x excess unphosphorylated peptides. However, our experiments with XPG protein have revealed critical limitations to this strategy for application to large proteins. Using the conditions developed for model phosphopeptides did not yield detectable MS signals for tryptic digests of XPG from a 1D gel band. This could be due to the low abundance of phosphorylated XPG and therefore the lower population of phosphorylated peptides in the mixture of tryptic digests. In addition, the small volume of the NTA chip channels and limitations of slow kinetics associated with surface capture might contribute to the problem. We are currently evaluating alternative IMAC methods including off-line NTA Ziptip minicolumns and on-line IMAC column interfaced with the mass spectrometer.

In parallel, we have identified several in vivo phosphorylation sites for endogenous XPG after its immunoprecipitation from whole HeLa cell lysates. An SDS-PAGE band containing $\sim 100 \mathrm{ng}$ of XPG from 2 liters of HeLa cells was cut out, digested by trypsin, and analyzed by MS. We sequenced over 40 tryptic peptides, generating $43 \%$ coverage of the XPG sequence, and identified four individual phospho-sites in the R-domain. DNA-PKcs was implicated as a candidate kinase for XPG by several lines of evidence, including MS identification of DNAPKcs as an interaction partner of XPG in the cell. We mapped two sites phosphorylated by DNA-PKcs in a critical subdomain of XPG. The in vitro phosphorylated construct was separated by PAGE, digested with trypsin or chymotrypsin, and analyzed by tandem MS. We had $90 \%$ coverage of the total sequence and identified two new in vitro phopho-sites at serine residues. Both serines are evolutionarily well conserved, and interestingly the second is also a predicted site for the CK2 kinase, suggesting the possibility of multiple layers of regulation.

We are continuing to develop alternative and improved strategies for identification of phospho-sites in native XPG. The goal is to understand regulation of XPG functions by cellular damage signaling pathways through phosphorylation and other post-translational modifications and to test the hypothesis that these provide a set of molecular switches controlling the activities, sub-nuclear localization, and protein partnerships of XPG. 


\section{Dynamic Reorganization of Chromosome Architecture During Meiosis Principal Investigator(s): Abby Dernburg}

Project Description

The purpose of this project is to develop tools and methods to further our understanding of large-scale chromosome dynamics and how they are linked to essential aspects of genetic transmission, including crossover distribution and chromosome segregation during meiosis. The Specific Aims, as outlined in the original project proposal, are as follows:

1. Visualize and quantify the dynamic properties of meiotic chromosomes.

2. Probe the role of chromosome architecture in controlling the distribution of crossovers.

3. Investigate the essential role of the cis-acting meiotic "Pairing Centers" and associated proteins

Accomplishments

Aim 1: we have developed a set of powerful cytological markers that enable us to stage meiotic chromosomes in a quantitative way. Specifically, we identified protein components of meiotic chromosomes that are present at discrete stages in meiosis, and we raised polyclonal antibodies against several important factors. These include HTP1 and HTP-3, two essential components of the axial elements, and several chromosome-specific markers for the meiotic Pairing Centers (see Aim 3). These tools are been used for a number of projects in the lab.

Aim 2: Peter Carlton, a postdoctoral fellow, has discovered an important link between crossover regulation and the dynamic organization of meiotic chromosomes. Cytologically, we and others have observed that meiotic nuclei transition through three major stages of prophase: the "transition zone," where homolog pairing occurs and synapsis is initiated, a distinct intermediate stage we have termed "early pachytene," characterized by asymmetric distribution of the chromosomes in the nucleus the completion of synapsis, and "late pachytene," where chromosomes have fully synapsed and are distributed evenly over the nuclear periphery. In a number of mutant conditions, the "early pachytene" stage is greatly extended at the expense of the population of late-pachytene nuclei. Pete has investigated the molecular basis for this delay in early pachytene, and found that it depends on the presence of meiotic double-strand breaks as well as DNA repair proteins specifically required for crossover recombination. Taken together with other information, this indicates the existence of a surveillance mechanism that detects crossover intermediates and delays meiotic progression until each chromosome has achieved a crossover. In addition, he found that this delay in early pachytene can be triggered by chromosome-specific synapsis defects, and in such cases it corresponds with a disruption of normal crossover control on the other chromosomes. This study has now provided the first direct evidence for a link between nuclear architecture and crossover control during meiosis.

Aim 3: This aim of investigating the role of the cis-acting Pairing Centers has become a major focus of several lab members, and is also the primary topic for significant follow-on funding that was obtained from the NIH. Through analysis of genetic interactions with Pairing Center mutations, we have identified the first family of meiotic proteins that interact with specific chromosomes. These include HIM-8, a protein specifically required for Xchromosome synapsis and segregation, and 3 paralogs that are responsible for autosomal synapsis and segregation. We have raised specific antibodies against each of these related proteins. A remarkable finding was that these protein factors not only bind to the cis-acting Pairing Centers but also associate with the nuclear envelope, and we are currently investigating the molecular basis for this attachment. In addition, we recently discovered an unexpected role for the Pairing Centers in a novel meiotic checkpoint that leads to apoptosis of nuclei with unsynpased chromosomes. These discoveries have provided exciting launching points for future studies in the lab. 


\section{Functional Interpretation of Cancer Genomes}

Principal Investigator: Joe W. Gray

\section{Project Description}

The purpose of this project is to facilitate study of how genes and the proteins they encode interact in human normal and diseased cells. Generating this body of knowledge and using it to improve disease management is one of the next major challenges in biology and medicine. This is daunting because of the complexity of the interacting systems - ultimately, thousands of genes and gene products may be involved in important cellular processes such as growth, death, motility, angiogenesis and proteolysis. Advanced analytical and computational technologies developed around existing strengths in engineering, computer science, structural biology and biology at Berkeley Labs are being developed to address these problems. Our initial focus is on cancer. Specifically, we are developed a cancer analysis "system" comprised of dozens of cancer cell lines that mimic important aspects of the genetics and biology of primary tumors and that capture the person-to-person variability found in primary tumors.

\section{Accomplishments}

We have compared the genomic and gene expression characteristics in a breast cancer cell lines "system" comprised of 55 breast cancer cell lines with those found in primary human tumors. The concordance is generally high suggesting that the cell lines do carry approximately the same recurrent aberrations as do the primary tumors. In addition, the heterogeneity between cell lines is as high as primary tumors.

We have initiated the design and construction of a semi-automated system for high throughput analysis of molecular (DNA, RNA and protein) and cellular responses (motility, cell death, proliferation rate, pathway activity) to therapy. In this system, stock cell lines grown and maintained in a central cell culture facility will be transferred from flasks and seeded into smaller volume multi-well cell culture plates, transferred to a multi-plate incubator, fed and inspected daily and harvested as needed. Individual plates will be treated with selected therapeutic or experimental agents in order to study the resulting molecular a biological responses. Cell properties will be analyzed using an automated microscope and cellular contents will be extracted for analysis of RNA and protein levels. Computational approaches are being developed to manage and analyze the resulting data.

This LDRD research supported an application in response to the NCI Integrative Cancer Biology Program which resulted in the award of $\$ 13,000,000$ over a 5 year period to develop and validate a computational model of cancer-related signaling networks. Eventually, this information will be used to identify subsets of patients that will respond to new therapeutic agents. The NCI project will use the cell lines and technologies being developed under the auspices of this LDRD. 


\author{
Neuroimaging with Advanced Molecular Probes \\ Principal Investigator: William Jagust
}

\title{
Project Description
}

The focus of this project is to develop methods for the non-invasive quantitation of the dopamine system and methods for quantitating the development of brain amyloid. For the dopamine system, we plan to: (1) define the best method for quantitating dopamine metabolism using FMT. These studies will utilize arterial input functions in comparison to tissue and imagebased input functions. (2) investigate measurement of dopamine transporters (DAT) using $\left[{ }^{11} \mathrm{C}\right] \mathrm{d}$-threo-methylphenidate (MP). For the amyloid studies, we plan to use Pittsburgh compound B (PIB) labeled with $\left[{ }^{11} \mathrm{C}\right]$ in order to : (1) Compare uptake of tracer in a small group of $\mathrm{AD}$ patients and normal controls (2) Investigate tracer uptake in a group of normal older individuals and a group with mild cognitive impairment, and (3) compare tracer uptake to memory performance in normal older subjects. These studies will also entail tracer kinetic modeling to investigate the best method of quantitating tracer uptake in the brain.

\section{Accomplishments}

As far as dopamine studies, to date we have completed 14 studies of normal volunteers using FMT. The data reveal high signal to noise with good definition of dopamine-containing brain regions, including small structures such as the caudate nucleus and substantia nigra. The studies have permitted us to evaluate different ways of modeling the data, and we are convinced that useful quantitative information can now be obtained with minimal invasiveness. Specifically, using a protocol that requires only an intravenous injection of tracer with two hours of imaging, separated by a break to empty the bladder, we can obtain high quality quantitative data without the need for arterial blood sampling. We have compared a fully quantitative data analysis approach, using arterial blood sampling and metabolite correction, with semiquantitative approaches, using graphical (Patlak) methods and iterative least-squares curve fitting methods, as well as simple specific to nonspecific uptake ratios. The graphical and iterative leastsquares approaches both use a brain region with little specific activity, the cerebellum, as the input function. We have found that these two approaches yield more reliable findings than either the fully quantitative approach, which is technically complicated and quite variable, or the simple ratio approach, which also tended to be quite variable. We have also begun to evaluate different image acquisition parameters including $2 \mathrm{D}$ versus $3 \mathrm{D}$ emission acquisition. Images acquired in 3D require a lower dose of radiotracer and have produced high quality images with good signal to noise. In addition, we were able to apply the same kinetic modeling approaches to the 3D data with similar results as the 3D data. We plan to continue these studies in the coming year, and add $\left[{ }^{11} \mathrm{C}\right] \mathrm{d}$-threo-methylphenidate.

Amyloid imaging studies have not begun. Considerable developmental work on cyclotron targetry to produce $\left[{ }^{11} \mathrm{C}\right]$ has been performed, and we have begun to develop methods for labeling of precursors. In the coming year we intend to perfect the radiolabelling, synthesize the PIB precursor, and perform initial human studies. 
Project Title: Identification and Analysis of Determinants of Centromere Identity in Drosophila Principal Investigator(s)-Gary Karpen

Project Description:

Chromosome replication, condensation, and segregation are mechanisms used by organisms and cells to accomplish the transmission of genetic information. Knowledge of the basic biology of inheritance is essential if we are to understand the relationship between aneuploidy and birth defects or cancer progression, and to diagnose and treat these conditions. The centromere is central to the chromosome inheritance process, and is the minimal chromatin element that is sufficient to promote normal formation and function of the kinetochore, the structure that is responsible for mediating interactions between the microtubule spindle and the chromosome.

We want to understand how and why sites destined for centromere function are chosen, how this 'centromere identity' is propagated through replication and division, and how the centromere assembles the kinetochore. It is clear that centromere identity and propagation are regulated by epigenetic mechanisms, rather than primary DNA sequence. An excellent candidate for an epigenetic marking protein is CENP-A, a histone H3-like protein that is present only in centromeric chromatin, in widely divergent species. Genetic and cytological analyses have shown that CENP-A is both a structural and a functional foundation for kinetochore assembly and function. In order to gain insight into the mechanisms responsible for propagating centromere identity, it is necessary to understand how new CENP-A is incorporated into replicated centromeres. This project is focused on using Drosophila CENP-A (CID) as an entry point to identify the proteins and mechanisms responsible for the propagation of centromere identity.

\section{Accomplishments:}

We have made substantial progress in elucidating components involved in determining centromere identity. First, we have shown that overexpression of CID results in ectopic localization of this protein, recruitment of other kinetochore components, and chromosome fragmentation and mis-segregation. Thus, CENP-A can seed ectopic, functional kinetochore formation, providing further evidence for its role as an epigenetic determinant of centromere identity. These findings also have relevance to cancer biology, because they provide a direct demonstration that CENP-A overexpression and mislocalization recently observed in colon cancer cells is likely to result in mis-segregation and aneuploidy.

Second, we have shown that centromeric chromatin contains a pattern of histone modifications that is distinct from both euchromatin and heterochromatin. These results suggest that other epigenetic 'marks' may play a role in centromere identity or function, in addition to CENP-A, and that general histone modification proteins (e.g. acetyltransferases, methyltransferases and kinases) may be critical components of this process.

Third, we have also made excellent progress in identifying and studying proteins that play a role in CENP-A deposition and function. Biochemical and genetic screens for CIDinteractors have identified chromatin assembly and remodeling factors (e.g. CAFs, CHD1), as well as histone modification enzymes (e.g. SU(VAR)3-9, TRX, NEJ). The TRX histone methyltransferase is particularly interesting, because the modification it regulates $(\mathrm{H} 3 \mathrm{~K} 4 \mathrm{Me})$ is one we identified within centromeric chromatin (see above). In addition, we have just completed genome-wide RNA interference screen for genes that are necessary for CID localization at centromeres. We have identified 50 positives. Candidates identified in the genetic, biochemical, and RNAi screens are currently being analyzed for functional roles in CENP-A deposition, and centromere identity and function. 
Imaging Three-Dimensional Signaling Networks In Normal And Malignant Tissue Principal investigators: G. Steven Martin and Mina Bissell

\section{Project Description}

A typical mammalian cell contains somewhere between several hundred and a few thousand signaling proteins that are organized into a complex signaling network, processing external information and generating cellular responses such as proliferation, survival, motility and differentiation. The long-term goal of this project is to develop a genome-wide picture of the spatio-temporal organization of signaling networks in normal and malignant cells. The immediate goals are to develop and use fluorescence imaging methods to follow the activation, translocation and membrane interactions of key signaling proteins in normal and malignant cells growing in three-dimensional cultures that replicate the normal tissue environment.

Realization of these goals will require collaborations between groups with expertise in imaging and biophysical techniques, signaling biochemistry and tumor cell biology. In the first year of this project we have initiated a three-way collaboration between the groups of Martin and Bissell (LBNL-LSD and U.C. Berkeley) and the group of Prof. Yoav Henis at University of Tel Aviv. We have been focusing on the non-receptor tyrosine kinase Src, because this molecule is a central mediator of growth factor and integrin signaling, and on small GTPases of the Ras superfamily, because these GTPases are known to be involved in malignant transformation.

Accomplishments

We are currently using fluorescence recovery after photobleaching (FRAP) to obtain information on the dynamics of membrane and microdomain association in live cells. We have studied the interactions of wild-type and activated Src with the membrane. The major conclusions to date are: first, Src(wild-type) exchanges between cytosol and membrane whereas activated Src associates with membrane proteins and does not exchange; second, the lateral diffusion coefficient (D) calculated for Src(wild-type) is $0.56-0.8 \mu \mathrm{m}^{2} / \mathrm{s}$, similar to that of a lipid probe, whereas the lateral diffusion coefficient of activated $\operatorname{Src}$ is much lower $\left(0.2 \mu \mathrm{m}^{2} / \mathrm{s}\right)$; and finally, a significant fraction of activated Src (but not wild-type Src) is immobile, possibly because of associations with adhesions. We are currently examining the role of the Src SH2 and SH3 domains in the protein-protein interactions that restrict Src mobility.

Oncogenic transformation by activated Src results in formation of invasive adhesions termed podosomes. To approach the function of Rho[GTP] in podosome formation, we have developed a high-resolution affinity-fluorescence technique to determine the subcellular localization of endogenous Rho[GTP]. The method employs GST-Rhotekin fusion protein as a soluble staining reagent to visualize active Rho in fixed and permeabilized cells. Strikingly, we observed Rho[GTP] to be localized at F-actin-rich podosomes in Src-transformed fibroblasts. To determine whether Rho function is required for the assembly or function of podosomes, we inhibited Rho function either by transient transfection with N19Rho or by treatment with the bacterial C3 toxin. Rho inhibition led to a dramatic decrease in the appearance of podosomes. Furthermore, inhibition of Rho also led to a dramatic decrease in the ability of the transformed cells to degrade the extracellular matrix, as judged by plating on a fluorescent matrix (FITCgelatin). Thus localized activity of Rho is necessary for the assembly and function of structures implicated in tumor cell invasion. 


\section{Systems Biology: Biological Input-Output Devices Principal Investigator: I.S. Mian}

\section{Project Description}

The aims of this project are to develop and apply mathematical, statistical, and computational models to assist in investigating biological systems. Novel and/or enhanced datadriven and conceptual models of heterogeneous sources of biological data will be formulated using general purpose frameworks such as probabilistic graphical models and kernel-based methods. Realising these models will necessitate exploring ideas from the field of (robust) optimization. The ensuing techniques and tools will be employed to study ageing, cancer and the origins of tissue specificity. The types of data to be examined include molecular sequences (DNA, RNA, protein), molecular profiles (transcript/gene, protein, metabolite) and biomedical corpora (text, image-text).

\section{Accomplishments}

A variety of novel and extant models have been explored and applied to illustrative and real-world problems. Examples of such models include hidden Markov models, robust sparse hyperplanes, Latent Dirichlet Allocation models, ensembles of finite mixture models, and large sparse graphs. The biological problems addressed encompassed questions related to telomere biology, breast cancer, melanoma, RNA editing in trypanosomes, and chromatin structure. The fruits of the research have been, are and will be of interest to researchers in computational and experimental biology. 


\section{Characterization of Adult Stem Cell Involvement in Mammary Gland Development Principal Investigator (s) Ortiz de Solórzano, Carlos}

\section{Project description}

Our principal goal is to study whether partially differentiated bone marrow adult stem cells can undergo transdifferentiation into epithelial stem cells and participate in the development of the mouse mammary gland. To achieve this goal we will perform same-species transplantation of bone marrow from GFP-expressing transgenic mice into wild type female mice with surgically cleared mammary fat pads. If transdifferentiation occurs, we expect to see green (GFP expressing) cells forming part of ducts of mammary outgrowths obtained after implanting epithelial tissue into the cleared fat pads.

A secondary goal of the project is to quantify the presence and distribution of native epithelial ASC's cells in the mouse mammary gland by using their known ability to retain DNA-binding compounds such as bromodeoxyuridine (BrdU). In this case, we will provide a continuous two week long supply of BrdU by implanting osmotic pumps into premenopausal females. After a nine week long clearing period, during which non stem cells will halve their BrdU on each cell division, the glands will be analyzed for the presence of BrdU retaining cells. This will be done using immunofluorescence with anti-BrdU antibodies. We will use an existing computer assisted microscopy system to measure the location and distribution of BrdU retaining cells in the mammary glands.

Accomplishments

Fixation tests results:

GFP is a highly soluble protein and is not effectively retained in tissue sections or cells that have lost their membrane integrity. We tested various methods of fixation on frozen mouse mammary gland tissue. We compared intensity of the fluorescence, leaking of the GFP and morphology of the tissue (H\&E). Out of the three repeats that we conducted using different fixation methods, formalin+sucrose and paraformaldehyde+ sucrose fixed GFP signals the best at 2 hours. However, formalin+sucrose was the best fixative because it preserved both the overall intensity and morphology of the GFP signal.

Transdiferentiation results:

When the mammary gland outgrowths were examined, only isolated green cells could be seen interspersed with the ductal epithelium, meaning that transdiferentiation does not occur, at least in the conditions used in our experiments. To further support this argument, all green cells expressed markers of hematopoietic lineage descent and therefore were not epithelial.

BrdU retaining results:

Concerning the characterization of the distribution of long term label retaining cells (LRCs) in the mouse mammary gland, a number of accomplishments have been done in the last twelve months. A protocol for double immunofluorescence staining of bromodeoxyuridine (used to identify the LRCs) and an additional marker on thin paraffin sections was developed. An algorithm for automatic segmentation of morphological structures in mammary tissue was implemented in order to speed up the reconstruction process (used to identify the areas where the LRCs are present). Finally, a method for $2 \mathrm{D}$ and $3 \mathrm{D}$ spatial analysis of tissue samples from serial sections was developed and integrated into our computer-based 3D microscopy system. Using this method we expect to quantitatively determine the pattern of distribution of LRCs and their spatial relationship with other cell populations. 


\title{
Materials Sciences Division
}

LB04001

\author{
Properties of Nanocrystals Under Extreme Conditions \\ Principal Investigator: Paul Alivisatos
}

\section{Project description}

In this project, we investigate properties of colloidal semiconductor nanocrystals under high temperature and pressure created by the absorption of intense ultrashort laser pulse. Using intense pulsed laser light, extreme conditions such as high temperature and pressure can be created through the energy relaxation of dense photo-excited electron hole pairs. Due to the short temporal width of laser pulses, the nanocrystals can be heated for a long duration compared to the time required for atomic rearrangements within a nanocrystal, but only for a short duration compared to diffusion of atoms between nanocrystals. As a consequence we are able to observe the properties of nanocrystals subjected to high temperatures and pressures without complication such as ripening and agglomeration.

\section{Accomplishments}

We investigated photoionization properties of colloidal semiconductor nanocrystals subject to intense femtosecond ultraviolet excitation. This allowed us to understand mechanisms and efficiency of photoionization when the number of excitations in each nanocrystal is very large (hundreds). Earlier studies on ionization of nanocrystals under weak excitation, where typically less than one exciton is created, indicated that ionization occurs via Auger scattering of electrons and holes. Our investigation showed that ionization occurs via resonant multiphoton absorption when the nanocrystals are under much stronger excitation condition. The efficiency of ionization via resonant multiphoton absorption is orders of magnitude higher than that from Auger ionization. We observed tens of electrons ionizing from each semiconductor nanocrystal of several nanometers in diameter.

We also investigated non-linear optical properties of semiconductor nanocrystals with high density of optically generated electron-hole pairs. Electron-hole pairs with initial densities of $10^{20}-10^{22} / \mathrm{cm}^{3}$ can be created with intense pulsed laser light, which subsequently relax in a time scale of a few picoseconds. We measured time-dependent behavior of second order optical non-linearity from the photoexcited semiconductor nanocrystals by recording hyper-Rayleigh scattering (HRS) signal. We found out that an increase in electron-hole density in CdTe colloidal nanocrystals enhances second-order electric susceptibility, $\chi^{(2)}$, leading to stronger scattering intensity (up to a factor of 4 larger) at second harmonic frequency. The increase of $\chi^{(2)}$ is, however, sub-linear to the density of electron-hole pairs, indicating the reduction of one-electron non-linearity with increasing electron-hole density. 
Molecular Control of Interfaces Between Biological and Synthetic Materials

Principal Investigator: Carolyn Bertozzi

Project Description

The integration of biological components into a synthetic device environment is a major frontier in the design of microscale and nanoscale devices. Living cells possess many capabilities that are desirable in a device context, as multi-enzyme metabolic conversions are useful for environmental bioremediation, energy harvesting, and industrial fermentation. They can also amplify and transduce signals in response to detection of soluble analytes, and thereby could function in biosensing devices. To achieve this, molecular control of the interface between the biological molecule or cell and the surrounding material is paramount.

The majority of intercellular communication events occur between molecules embedded in the plasma membrane. These molecules comprise a diverse collection of receptors and ligands that serve to anchor the cell to appropriate growth locations, receive communication from adjacent cells and soluble signaling molecules, and send information regarding the polypeptide synthesis occurring within. Through a variety of complex mechanisms, these molecules serve to relay environmental information to the cell interior, ultimately resulting in gene regulation and thus control of cellular behavior. The goal of this project was to develop a common molecular platform that can be used to interface living cells with synthetic materials in an interactive manner and to study the response of cells to their support material. These studies will provide essential information for the successful incorporation of cells into device structures.

\section{Accomplishments}

Supported lipid bilayers were chosen as the central material for these studies because they closely (and perhaps uniquely) mimic the two-dimensional fluid reaction environment corresponding to the surface of another cell. The last year of this LDRD has developed a modular chemical approach that can attach the soluble domains of cell surface proteins to supported lipid bilayers (SLBs) using site selective protein modification reactions.

This method has been developed in two parts. First, synthetic phosphatidyl ethanolamine derivatives bearing aminooxy groups were synthesized and incorporated into bilayers. Following this, two new chemical strategies were developed to introduce ketone binding partners on the surface of a wide range of protein targets. The first of these was a new metallocarbenoid-based tryptophan modification reaction developed in our lab, and the second was an oxidative decarboxylation reaction for the conversion of $N$-terminal aspartic acid residues to pyruvamide derivatives. The ketone moiety introduced using both of these techniques reacts rapidly with the aminooxy groups to form an oxime, thus attaching the proteins to the lipid surface through a robust linkage. When carried out with fluorescent protein substrates, both the protein attachment and the bilayer fluidity could be confirmed. This new tool is anticipated to increase the range of proteins that can be attached to SLBs dramatically, and thus the range of living systems that can be interfaced with them.

Another major development of the last year is a new technique for monitoring simultaneously, at the ensemble and single molecule level, both the redistribution of proteins associated with SLBs and cell adhesion to these molecules. As an example, we were able to probe the interaction between a non-neuronal neurexin (Nrx)-expressing cell and a SLB displaying GPI-neuroligand (Nlg). Distinct patterns of Nrx distribution were found to be associated with cell-bilayer adhesion. These results provide the basis for studying the interface of cells with modified surfaces at the level of clusters of receptor-ligand complexes. 


\section{Techniques of Sample Controls for a Transmission Electron Aberration-Corrected Microscope Principal Investigator: Ulrich Dahmen}

\section{Project Description}

The field of electron microscopy is undergoing a revolution based on major recent advances in electron optics, detectors, stage design and computing power. These advances make it possible to overcome the fundamental limitations currently imposed by lens aberrations. The ability to correct for these aberrations is about to transform the field of electron microscopy, and the TEAM construction project, led by NCEM on behalf of five DOE microscopy efforts (LBNL, ANL, BNL, ORNL and FS-MRL at UIUC) will initiate a new generation of electron optical instrumentation. However, in the process of surpassing traditional limits of instrumental resolution and sensitivity, new barriers often rise to the surface. It has been found that such limitations are frequently posed by the sample itself, the stability of the microscope site, the sample stage and the ability to quantify experimental data for accurate comparison with atomistic simulations.

The current project will address some basic scientific and technological issues to help lay the scientific foundation and prepare for the challenges posed by this new technology. The objective is to develop techniques for imaging, spectroscopy, samples, stages, data analysis and operating parameters under aberration corrected electron microscopy. To take advantage of the greatly increased sensitivity of aberration corrected beams it will be necessary to develop new sample preparation methods using innovative techniques of ion beam thinning with novel ion sources and geometries. It is equally important to begin evaluation of prototype aberration correctors and monochromators in the context of scientific research on the atomic structure of materials. Evaluations of these novel electron optical components will need to proceed in close collaboration with their designers. This is particularly important in these first stages of their development, where the integration of both components into the standard electron optical column is by no means straightforward, and is typically optimized for only a single application. A full appraisal of the limitations and benefits of each configuration for applications to materials research on defects and nanophases will be a crucial factor in this research.

\section{Accomplishments}

Two microscope STEM columns were secured from other laboratories and an interim site was prepared for instrument installation. Before transporting the column, a baseline test of the uncorrected instrument performance was conducted in cooperation with the designer of the aberration corrector. Installation at NCEM is currently underway. In parallel, an evaluation procedure for STEM imaging has begun on NCEM's new monochromated microscope. The performance of the monochromator in the spectroscopy mode has exceeded expectations, allowing the quantification of the confinement effect in individual CdSe quantum dots, and the identification of an alloy induced bandgap change in InAs/GaAs films. However, this work has also shown that the spatial resolution in the STEM imaging mode is compromised by the monochromator and requires redesign of the condenser optics.

Initial tests of a newly designed low-energy Ar ion gun inside a Focused Ion Beam instrument have shown promise for a new integrated approach to sample preparation from bulk material to thin foils suitable for atomic resolution imaging. Permanent installation of the new ion gun will proceed following the implementation of a newly-devised alignment procedure. 


\section{Directed Assembly of Germanium Island Arrays on Gold-Patterned Si (100) Principal Investigator(s): Oscar D. Dubón, Jr and J. Alexander Liddle}

\section{Project Description}

The past two decades have witnessed remarkable progress in the synthesis of semiconductors of reduced dimensions. Two-dimensional structures such as quantum wells and superlattices are readily produced from a wide variety of materials systems using thin-film deposition techniques as layer deposition can be controlled down to the atomic level. However, even the most advanced thin-film techniques such as molecular beam epitaxy (MBE) are not naturally suited for the synthesis of ordered island assemblies due to the inherent lack of control over growth in the in-plane directions. Despite considerable efforts to direct the assembly of zero- and onedimensional semiconductor elements (quantum dots and wires, respectively), one of the great challenges in nanoelectronics remains the development of synthesis and processing routes that will enable the realization of ensembles of semiconductor nanostructures of well-controlled size and arrangement across macroscopic length scales.

In this LDRD, we have pursued an integrated approach in advanced semiconductor epitaxial growth and nanolithography to form two-dimensional arrays of germanium islands. We have used the superior materials deposition control afforded by MBE in combination with the island site selectivity that can be achieved on patterned substrates to explore the growth epitaxial Ge islands in predetermined arrangements. Nanolithographically produced masks to form $\mathrm{Au}$ patterns on Si yield a precisely templated substrate for the subsequent deposition of Ge.

\section{Accomplishments}

We have achieved two-dimensional ordering of Ge islands. For a square array of Au dots, a square lattice consisting of Ge islands less than $100 \mathrm{~nm}$ in height has been realized (Figure 1). The island arrays extend over $10^{5} \mu \mathrm{m}^{2}$, an area limited only by the extent of the Au pattern. Surprisingly, the Ge islands grow away from the Au. Investigations are underway to understand the forces driving this remarkable process. This simple, versatile method to manipulate island growth processes that was developed through this LDRD opens a new path for directed islandarray assembly over large areas.

Successful demonstration of the directed growth of island arrays represents an important step toward the development of a technique for the growth of electrically interconnected semiconductor nanostructures.

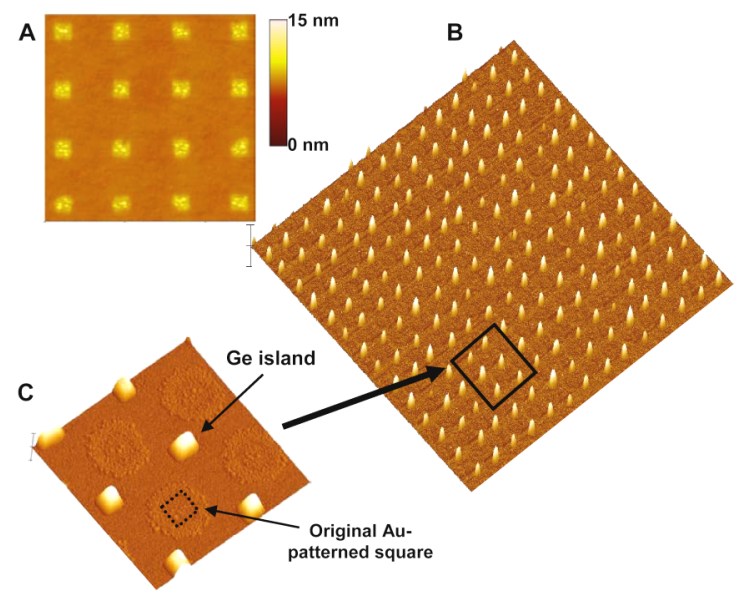

Figure 1. Atomic force microscopy images showing: (A) the Au-pattern after annealing at the growth temperature $\left(600^{\circ} \mathrm{C}\right)$ for 1 hour $\left(2.5 \times 2.5 \mu \mathrm{m}^{2}\right)$. (B) Ge deposition in the Au-patterned region, where Ge islands order across hundreds of microns (height scale: $100 \mathrm{~nm} ; 10 \times 10 \mu \mathrm{m}^{2}$ ). (C) A zoom-in (height scale: $100 \mathrm{~nm} ; 1.5 \times 1.5 \mu \mathrm{m}^{2}$ ) showing the relative position of Ge islands to the patterned Au squares 


\section{Development of Light-Switchable Potassium Channels \\ Principal Investigators: Dirk Trauner and Matthew Francis}

\section{Project Description}

Potassium channels are among the core features of life, occurring in virtually every eukaryotic and prokaryotic cell. Their ability to pass potassium ions through lipid bilayers and thus control the transmembrane potential underpins elementary cellular functions such as excitability, secretion and volume regulation. While their importance in neurobiology, physiology and medicine is evident, their potential in material sciences remains to be explored. This project is to develop artificial light-switchable channels using a strategy that combines chemical synthesis and molecular cloning. These devices could be produced in relatively large quantities and incorporated into artificial membranes or cells. Importantly, the independent mechanisms of voltage and ligand gating should not be affected by our proposed modification, allowing for logical operations to be performed with the photo-switchable channels.

A combination of molecular cloning techniques and chemical synthesis will be used to procure modified ion channel that can be gated by irradiation. Three different strategies for the development of light-switchable channels were considered. The first concerns the modification of cysteine residues that are genetically engineered into suitable positions on the extracellular surface of potassium channels. The second would utilize tyrosine residues as anchor points. The third strategy would involve modification of the gating mechanism of potassium channels..

\section{Accomplishments}

The primary goal of our proposed research has been fully realized. We have succeeded in rendering certain potassium channels sensitive to light. A small organic molecule that functions like a light-switch, termed MAL-AZO-AZ, was prepared by total synthesis and covalently mounted onto the extracellular surface of a potassium channel fine-tuned by site-directed mutagenesis. In its extended conformation, the molecule blocks the pore of the channel. Upon irradiation with ultraviolet light $(380 \mathrm{~nm})$, it undergoes a change in geometry that literally withdraws the blockade from the pore, restoring the conductance of the channel.

In collaboration with Richard Kramer (UC Berkeley), we have shown that this modified channel can be incorporated in cells (Xenopus oocytes) and switched between its blocked and unblocked state upon irradiation at different wavelengths. The magnitude of the blockade was found to be highly wavelength-dependent, reflecting the photostationary state of the light-switch. We are currently trying to directly measure the cis/trans ratio of the MAL-AZO-QA photoswitch as a function of the wavelength applied using NMR-spectroscopy. Furthermore, we are using single-channel patch clamp electrophysiology to gain detailed insight in the functioning of the channel and to follow azobenzene photoisomerizations on a single channel level.

Furthermore, we have shown that hippocampal neurons expressing these chemically modified channels can be reversibly silenced upon irradiation. This system should allow for the study of neuronal circuits with perfect temporal and spatial control and could ultimately lead to the development of an artificial retina.

In light of our success with the external cystine modification strategy, the other two components of the original proposal ("Tyrosine modification" and "internal modification of the gate") were not pursued. 
Nanoscale Lithography to Guide Self-Assembly for the Creation of Functional, Hierarchical Nanostructures

Principal Investigator(s): J. Alexander Liddle and Nitash Balsara

\section{Project Description}

The purpose of this project is to develop techniques for the controlled assembly of nanostructures. Length scales that are accessible with conventional lithographic techniques are limited to approximately $25 \mathrm{~nm}$ - much larger than many potentially useful structures such as nanotubes, nanocrystals or functional molecules. A method of integration is required to allow the imposition of a structural hierarchy and interconnection between the various elements in order to realize the full potential of many nanoscale objects. Guided self-assembly provides a means of addressing this issue. The techniques explored through this research will enable the development of a "toolkit" based on lithographically guided self-assembly suitable for bridging the gap between current lithographic methods and the nanoscale.

The research will address simultaneously the areas of lithography and materials a deliberately synergistic approach - in order to make significant progress. Two model systems were targeted initially: a ferrocene/styrene or ferrocene/isoprene blend and a plyethyleneoxide/polyisoprene diblock copolymer. These materials will be deposited on substrates patterned lithographically to exhibit different interactions with the different component materials, thus controlling their spatial distribution. The conditions necessary to produce spatial frequencies significantly higher than those of the initial lithography will be investigated. The potential for developing materials that are themselves functional when so structured, or that act as templates to control the distribution of other functional materials, will also be explored.

\section{Accomplishments}

One of our most significant accomplishments has been the development of a simple and reproducible method of forming surfaces patterned with areas of controlled hydrophobicity using UV/ozone exposure of organo-trichlorosilane self-assembled monlayers. We have also developed synthesis methods for tri-block systems that, after some experimentation, can be spin-coated and that do not dewet upon annealing. The synthetic target was poly(styrene- $b$-isoprene- $b$-methyl methacrylate). As both the styrene and methyl methacrylate blocks have high glass transition temperatures $\left(\sim 100^{\circ} \mathrm{C}\right)$ dewetting is less of a problem than in the systems we have investigated previously. A sample of poly(styrene- $b$-isoprene- $b$-methyl methacrylate) has been synthesized with $\mathrm{M}_{\mathrm{w}}$ $\approx 150,000$ and $\phi_{\mathrm{S}}=0.24, \phi_{\mathrm{I}}=0.27, \phi_{\mathrm{MMA}}=0.48$.

The second phase of the project, currently underway, is to determine the conditions under which thin films of this material will self-organize on substrates with patterned surface energies. We have already developed the appropriate imaging techniques in both scanning electron and atomic force microscopy to characterize the structures developed and the electron beam lithography and processing methods to generate features at the $25 \mathrm{~nm}$ length scale. 
Extending Electron Delocalization in Mixed-Valent Molecular Assemblies Principal Investigator: Jeffrey Long

Project Description

Considerable effort has been devoted to the synthesis of conjugated organic oligomers as potential conducting elements in future molecular electronic devices. Many of the direct checks for electronic communication, however, show the conductivity in these oligomers to drop off rapidly with chain length. For example, cyclic voltammograms of metal-terminated oligoalkynes of the type $\operatorname{Re}(\mathrm{C} \equiv \mathrm{C}){ }_{n} \operatorname{Re}$ reveal a steady decrease in the splitting of the rhenium-based redox waves with increasing $n$, until they merge at $n=10$. An unexplored means of possibly avoiding such a drop in conductivity is to construct oligomers containing periodically-spaced redox-active metal sites as electron-hopping stations. To minimize any energetic preference of an electron (or hole) for a particular metal site, one would like all of the metal centers to have essentially identical coordination environments. As a test of this hypothesis, we have explored the synthesis and characterization of symmetric molecular assemblies containing three or more equivalent ruthenium centers connected through pyrazine or bior tripyridyl ligands.

Previous work had shown that a mixed-valent pyrazine-bridged square was formed according to the following reaction (cyclen $=1,4,7,10$-tetraazacyclododecane).

$$
4\left[(\text { cyclen }) \mathrm{RuCl}_{2}\right]^{+}+4 \mathrm{pz}+3 \mathrm{e}^{-}->\left[(\text {cyclen })_{4} \mathrm{Ru}_{4}(\mathrm{pz})_{4}\right]^{9+}+8 \mathrm{Cl}
$$

Cyclic voltammetry measurements on a solution of the product revealed a reduction wave at $E_{1 / 2}=-0.472 \mathrm{~V}$ versus $\mathrm{FeCp} 2{ }^{0 /+}$ and a series of three poorly-resolved oxidation waves at $E_{1 / 2}=0.051,0.171$, and $0.345 \mathrm{~V}$, suggesting a Class III mixed-valence behavior.

Accomplishments

The longer linear bridging ligand 4,4'-bipyridine is generally observed to be less effective than pyrazine at propagating electron transfer. To check if this would also be true in a square geometry, reaction 1 was carried out under identical conditions using 4,4'-bipyridine in place of pyrazine. Unexpectedly, however, the slight flexibility of this longer ligand was instead found to prompt formation of a molecular triangle:

$$
3\left[(\text { cyclen }) \mathrm{RuCl}_{2}\right]^{+}+3 \mathrm{bpy}+2 \mathrm{e}^{-}->\left[(\text {cyclen })_{3} \mathrm{Ru}_{3}(\text { bpy })_{3}\right]^{7+}+6 \mathrm{Cl}
$$

The product consists of three equivalent ruthenium centers arranged in an equilateral triangle and linked via gently bowed bpy ligands. Here again, autoreduction of ruthenium during the reaction leads to a mixed-valent charge state. Remarkably, cyclic voltammetry measurements revealed this charge state to have an even greater stability range than that observed for $\left[(\text { cyclen })_{4} \mathrm{Ru} 4(\mathrm{pz})_{4}\right]^{9+}$. Although fully reversible redox behavior was not observed, the separation of more than $1 \mathrm{~V}$ between the first reduction and oxidation waves indicates an inferred comproportionation constant of $K_{\mathrm{c}} \sim 10^{20}$. This extraordinary result, which suggests a Class III system despite the elongated bridging ligand, can be reconciled by considering the constraints placed on the bridging ligands by the triangular geometry. Most notably, it forces the two pyridine rings in each bpy ligand to be coplanar, while at the same time ensuring that their vacant $p$ orbitals are interacting with the same $\mathrm{Ru} 4 d_{x y}$ orbital as the neighboring ligand. Accordingly, DFT calculations confirmed the strong mixing of Ru- and bpy ligand-based orbitals.

Attempts to generate mixed-valent cubes containing eight ruthenium centers bridged through pz or bpy have not yet led to a pure crystalline product. 
Modeling Quantum Coherence and Transport in Nanoscale Spin, Charge, and Flux Devices

Principal Investigator: Joel E. Moore

\section{Project Description:}

This project will start a new theoretical research effort in nanoscale devices and quantum coherence. Problems to be studied include the creation and control of quantum entanglement in systems with coupled degrees of freedom, and the electronic and mechanical properties of singlemolecule and single-electron transistors. One primary scientific goal is to find a theoretical explanation for recent experimental results indicating that vibrational excitations strongly influence transport in single-molecule transistors. Another goal is to understand causes of decoherence in coupled spin and flux devices for quantum computing. Successful theoretical modeling of nanoscale electronic devices will eventually require a combination of analytical techniques and ab initio computational methods. This combination has proved very useful in traditional semiconductor electronics. Molecular electronics are at an early stage, and for some devices there is not even a qualitative understanding of their properties.

The majority of the proposed research will be analytical and aimed at developing and studying basic models for experiments that are not yet understood. Specific methods include the Keldysh and Landauer descriptions of nonequilibrium transport and the Caldeira-Leggett approach to dissipative systems. Once testable models are developed, it may be desirable to refine these models using high-performance computation.

\section{Accomplishments:}

The first topic from last year's report, electron-phonon coupling in molecular electronics, has been extended in two directions. The first is to understand phonon transport in extended one-dimensional structures like semiconductor nanowires, in which quantum mesoscopic effects are detected in existing experiments. The second is to model how molecular devices can be used as "phonon filters" in thermoelectric devices.

The results from the first year of the project on superconducting arrays, detailed in last year's annual report, have been extended to include a theory of quantum fluctuations in Josephson-junction arrays made from unconventional superconductors like Sr2RuO4. Such superconductors have a coupling between spatial geometry and superconducting physics because of the nontrivial symmetry of the order parameter, and are especially interesting when the superconductor breaks time-reversal symmetry. Fabrication of functioning superconducting arrays has currently not been possible for these unconventional superconductors, but we have also worked on understanding dissipation effects in conventional superconducting arrays and films, which can change the nature of the superconductor-insulator phase transition. With follow-on funding, we are also continuing studying interaction effects on spin transport in semiconductors with Dr. J. Orenstein of LBL as these effects are of fundamental importance in realistic spintronic devices close to room temperature. 


\section{Magnetism at the Nanometer Scale in Spin Polarized Materials \\ Principal Investigator: Yuri Suzuki}

\section{Project Description}

In this program, our major objective is to develop a fundamental understanding of the role of surfaces and interfaces on the nature of magnetism in highly spin polarized thin film material. The nature of magnetism at boundaries of spin-polarized materials is a fundamental issue that has yet to be fully understood and may place technological limitations on the implementation and performance of spin-polarized devices. Through direct electron beam patterning and novel nanoimprinting methods, we will probe the relationship among structure, surfaces and magnetism in geometrically confined islands of doped perovskite manganites.

Nanoscale islands of doped perovskite manganites are a model system in which to study the relationship among structure, surfaces and magnetism. In particular, we will: (i) develop an imprint lithography process for the nanofabrication of a templated oxide substrate for half metallic oxide structures; (ii) fabricate $100 \mathrm{~nm}$ and sub $100 \mathrm{~nm}$ islands of single crystalline half metallic materials by direct electron beam patterning; (iii) perform structural characterization of the island material by $\mathrm{X}$-ray diffraction, atomic force microscopy and transmission electron microscopy in order to evaluate crystalline quality, surface morphology and microstructure; (iv) perform magnetic magnetization of nanoscale islands in order to understand magnetic domain structure, magnetization reversal and magnetic anisotropy in nanoscale islands where surface effects are dominant; (v) compare experimental results with those of micromagnetic simulations. Accomplishments

We have been successful in fabricating submicron size islands of magnetic oxides by using conventional optical lithography and more recently by electron beam lithography to pattern blanket epitaxial films. In optical lithography, we are limited to islands whose diameter $(>500 \mathrm{~nm})$ is at least ten times larger than its height. In such islands, the magnetics is dictated by the strain due to the lattice mismatch between the film and the substrate. Patterning of epitaxial thin films into islands by electron beam lithography has proven to be extremely challenging since Ar ion milling is the only method for removal of the magnetic oxides. Electron beam lithography was used to define a pattern in the electron beam resist, then etched into an intermediary metal mask and then finally transferred to the magnetic oxide material.

Another notable recent accomplishments has been to demonstrate layer-by-layer growth of these doped manganites using pulsed laser deposition where stoichiometric transfer of material from target to substrate is readily accomplished. The oscillations in the anti-Bragg peak of the [001] are direct evidence of the layer-by-layer growth that agrees with simulations.

We have also pursued the route of depositing magnetic material on patterned substrates. We have performed experiments of depositing oxide as well as $\mathrm{CoPt}$ multilayers on patterned silicon substrates. The CoPt multilayer islands exhibit single domain behavior. The oxide islands, which are in a polycrystalline state on silicon, exhibit ferromagnetism but their domain evolution is difficult to image by magnetic force microsopy (MFM) and photoemission electron microscopy (PEEM).

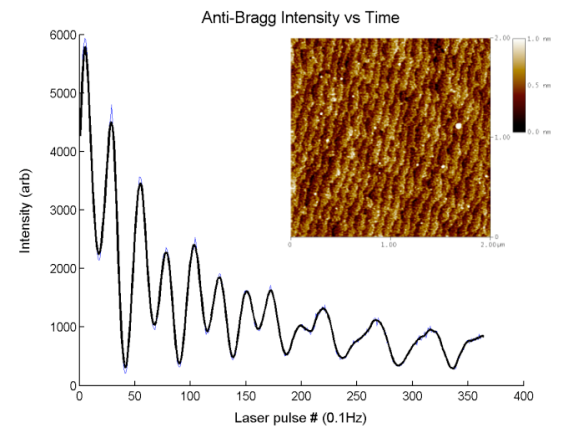

Figure 2. Growth oscillations of the anti Bragg peak of the $[00 \mathrm{~L}]$ measured on $(\mathrm{Pr}, \mathrm{Ca}) \mathrm{MnO}_{3}$ film on $\mathrm{SrTiO}_{3}$ substrate. The inset shows smooth terraced growth of the film using atomic force microscopy. 


\title{
Nuclear Science Division
}

\author{
Effective Field Theory and Few-Nucleon Systems \\ Principal Investigator: Paulo Bedaque
}

\section{Project Description}

The goal is to extend the effective field theory methods recently developed for the two-nucleon sector to systems involving three or more particles (including bulk matter). We also intend to explore the possibility of first principles calculations of the parameters of the effective theories in order to establish a direct link between the standard model of particle physics and nuclear phenomenology.

\section{Accomplishments}

Nuclear physics calculations have, up to now, been done at a macroscopic level. This is true for the phenomenological approach using empirical nucleon-nucleon potentials and for the effective theory (EFT) approach we helped to develop in the last two years. In EFT, the information about the interaction between nucleons is encapsulated in the value of a few constants that have to be determined using experimental data. On the other hand, Quantum Chromodynamics (QCD) is known to be the true microscopic theory describing not only those interactions, but also the nucleon and pion masses and couplings. A fundamental goal of theoretical physics is then to be able to use QCD to predict the value of nucleon and pion masses and couplings, establishing a bridge between the QCD and nuclear phenomena. Besides the purely theoretical interest in such a work, this connection will allow, in the future, the clean computation of quantities of phenomenological interest for which experimental information is difficult to obtain.

The main obstacle for such a project is that QCD in the low energy regime we are interested in is a non-perturbative theory. Presently, the only method known of general applicability in this regime for first-principle calculations is lattice gauge theory. Despite being about 30 years old, three recent developments made it possible to use it in nuclear phenomena: the use of "improved actions", the invention of quark discretizations with perfect chiral symmetry and the continued increase in computer power. In the last year we started a program to pursue this kind of calculation. First, we identified some problems that any lattice calculation of nuclear forces would face. The large scattering length between two nucleons shifts the energy levels of two nucleons in a finite lattice to unphysical regions for any lattice smaller than 8 fermi. We subsequently found a way around this issue by using unusual boundary conditions and the "Aharonov-Bohm" effect. Through this method much smaller lattice sizes (4-5 fermi) can be used. Considering that the computational cost of these calculations scales roughly with the third power of the (spatial) lattice size, this brings these calculations within the realm of the possible. Subsequently, we used effective theory techniques to show that a much larger reduction in computer time can be obtained since the unusual boundary conditions can be applied to the "valence" quarks only.

In the meantime we started to "tool up" on the software and hardware required to actually perform these computations in practice. A small collaboration was formed (Nuclear Physics with Lattice QCD, NPLQCD) that, after some preliminary studies performed in the small Beowulf cluster of the Nuclear Theory Group, submitted proposals for substantial computer time to the SciDAC project. 


\section{Research and Development for Double Beta Decay Experiments Principal Investigator: Kevin Lesko}

\section{Project Description}

With the success of the Sudbury Neutrino Observatory in convincingly demonstrating neutrino transformations, resolving the Solar Neutrino Problem, and also proving that neutrinos have mass, and with KamLAND's refinement of the oscillation parameters, we are faced with several fundamental questions about neutrinos: the absolute mass of neutrinos (the oscillation experiments have measured the differences in masses between the species), the ordering of the neutrino masses between the three known neutrino flavors (the mass hierarchy), and the exciting prospect of determining if the neutrino is its own antiparticle. This characteristic of a massive particle being its own antiparticle would make the neutrino unique. Neutrinoless double beta decay ( $0 v \mathrm{DBD})$ experiments can determine the absolute neutrino mass, the hierarchy of the neutrino masses, and, most importantly, provide the only technique to determine if the neutrino is its own antiparticle. The importance of double beta decay has been recently highlighted in the American Physical Society's Neutrino report (www.aps.org) stressing that next generation DBD experiments are the highest priority for the US neutrino community.

The purpose of this project is to develop key technologies, data analysis tools, and comprehensive simulation packages for the next generation of $0 \mathrm{vDBD}$ experiments. There are several leading candidates for the next generation experiments, one using Ge-diodes to detect the energy as ionization (Majorana), and another using $\mathrm{TeO}$ cryogenic crystals to detect the energy as heat (Cuoricino/CUORE). Because of the exceedingly long lifetimes for 0vDBD, radioactive backgrounds are the major obstacle for these experiments. The Ge experiment draws on nearly four decades of experience operating Ge-diodes. The Te cryogenic technique has made great progress in the past decade in developing and operating small-scale arrays of $\mathrm{TeO}$ crystals. However, controlling and identifying backgrounds remain the greatest challenge. A variety of methods have been explored to reduce the sensitivity of the experiments to backgrounds through the physical reduction of the radioactive backgrounds, by investigating novel detector designs and operating procedures, and by developing comprehensive simulation packages. We are developing data analysis strategies and tools to reduce and interpret data from the experiments.

\section{Accomplishments}

For the Majorana collaboration we are developing and maintaining the comprehensive Monte Carlo simulation package MJSIM. Our efforts have predominantly focused on using modern simulation tools from the high energy and nuclear physics communities to assemble an experimental simulation package that can be shared with the entire collaboration. This simulation package handles the detector geometries, media properties, shielding characteristics, and important physics interactions. We are developing novel detector segmentation schemes and data analysis capabilities to reduce the sensitivity of the experiment to internal and external backgrounds. To better understand the irreducible backgrounds, we have initiated studies into the feasibility of building Ge crystals in underground environments.

For the Cuoricino project we have divided our efforts between 1) data analysis techniques, 2) research in producing thermistors, and 3) investigating TeO crystal production and contamination. Thermistor production saw significant progress in measuring the neutron dose needed to create the thermistors. 


\section{Research on a Next Generation Vertex Detector \\ Principal Investigators: Howard Wieman and Howard Matis}

\section{Project Description}

The purpose of this work is to develop an improved vertex detector technology suitable for the next generation experiments at RHIC, heavy ions at LHC and NLC. Upon completion of the LDRD, we will have enough information to write a proposal to build a full-size detector.

Accomplishments

We completed a study on the effect of varying the pixel pitch. We built and tested a sensor with 5, 10, 20 and $30 \mu \mathrm{m}$ spacing. Our tests show that to first order the charge collection was identical when comparing the central pixel to the charge collected on its neighbors. As the pixel spacing decreases, more total charge is collected. This observation can be explained by the fact that, as pixel spacing becomes smaller, the charge is collected by the diode faster, so there is less time for it to recombine. This result implies that we can easily extrapolate our measurements at $20 \mu \mathrm{m}$ spacing to a detector of different size.

To explore how charge is collected on the position, we tested a sensor at LBNL's National Center for Electron Microscopy. Using the scanning electron microscope, we have been able to position the beam on a pixel with a precision of about $1 \mu \mathrm{m}$. This allows us to explore algorithms for determining the position of an incoming particle by relating how charge is shared on neighboring pixels.

We have fabricated and analyzed photogate technology as a possible way to simplify correlated double sample noise reduction and reduce charge spreading and found that the undesirable signal delay is probably consistent with surface traps at the $\mathrm{SiO}_{2}$ silicon boundary.

Two generations of "active reset chips" have been fabricated and partially tested. In this approach the pixel voltage is reset to the empty level with a feedback amplifier, potentially reducing fixed pattern noise and the KTC noise associated with a passive reset switch. Preliminary testing shows some noise reduction, but not the full potential improvement.

We have also been collaborating with colleagues at UC San Diego on the suitability of using an APS sensor in an electron microscope. We have been studying the response of electrons from energies of $100 \mathrm{keV}$ to $300 \mathrm{keV}$. Results show that single electrons can be detected with a good signal-to-noise ratio. This technique is well suited for this application, overcoming problems with traditional CCDs that suffer radiation damage.

Part of our R\&D effort this year has been directed toward developing a vertex detector design based on APS pixels developed by the LEPSI/IReS group in Strasburg. Progress has been made on a high-speed, low-noise readout. Mechanical work has demonstrated construction techniques for ultra-thin designs using thinned silicon. 


\section{Physical Biosciences Division}

LB03006

Microscopic Imaging in High-Throughput Screening for Crystals of the Bacterial Ribosome

Principal Investigator: Jamie H. Doudna Cate

Project Description

The determination of macromolecular structures by x-ray crystallography critically depends on obtaining well-diffracting crystals. The explosive growth in bioinformatics has dramatically increased the number of interesting problems in structural biology, especially those that involve supramolecular complexes that reach many millions of Daltons in molecular weight, such as the ribosome, spliceosome, DNA replicase, and RNA polymerase. Recently, structures of ribosomal subunits and the intact ribosome, as well as of RNA polymerase, have been determined at medium or high resolution. However, these monumental achievements highlight the fact that the available biological material for crystallization trials is limited and costly to produce.

The goal of this project is to develop microscopic imaging tools to facilitate screening for crystals of the bacterial ribosome in different functional states in a high-throughput manner. Liquid-handling robotics are increasing the number of crystallization trials we are presently conducting in the laboratory. Yet, it has not been possible to adequately document and quantify the results of crystallization trials in a systematic way. Moreover, as the number of crystallization trials increases, and the volume of each trial shrinks, it will be critical to automate the imaging of each experiment as a function of time. The tools developed in this work will be widely applicable to the structural study of many other supramolecular complexes at the center of key biological processes.

Accomplishments

Our most significant accomplishment has been to obtain well-diffracting crystals of the entire bacterial ribosome. These crystals diffract x-rays to atomic resolution, and will provide the first structure of the intact protein synthesis machinery. Obtaining crystals of this quality was the principle aim of the project, and was to be aided by the development of crystallization automation technologies. We have made significant progress in overcoming the bottleneck of manual experimental setup by means of liquid-handling robotics and rapid protocols for using multi-channel pipettors. However, we discovered a number of issues that must still be overcome to automate microscopic imaging of each crystallization trial at $4^{\circ} \mathrm{C}$, primarily at the level of the robustness of electronic components in cold and humid environments. These issues will be tackled in future experiments. 


\section{High-throughput Production of Proteins and Protein Complexes Principal Investigator: Thomas Earnest}

Project description

This proposal initiated a program in technology development for the production of proteins and protein complexes in the quantities necessary to pursue biological studies on these samples. This includes the expression of single polypeptide chains along with the co-expression of protein-protein complexes when biologically-relevant interacting partners are identified. The automation of this process will be pursued in order to provide a high-throughput platform for systematic biological studies. Integral membrane proteins will be attempted once the technology for soluble proteins is in place and made adaptable to the added difficulty of selecting expression systems and purification methods optimal for automating the production of this challenging set of proteins. We also will explore the use of artificial intelligence methods to analyze and optimally control these experiments, and their information flow.

To attempt a genomic-scale production of proteins and protein complexes, methods and technologies need to be developed that allow for the determination of which constructs and sets of constructs will produce large amounts of protein in the soluble fraction for purification, or can be refolded. Searching for the correct constructs or sets of constructs would benefit greatly from the development of parallelized screening methodologies and their implementation into an automated and intelligently controlled instrumentation environment, as would a multi-tiered approach to the production of each target and target complex. We will implement these methods on laboratory automation as part of this project, such as the TECAN Genesis liquid-handling robot and HIGRO multichamber incubator/shaker. In future effort we will extend this to more optimal instrumentation that can be well integrated into a production pipeline, while maintaining the flexibility required for the range of samples to be produced. Other approaches, such as the modulation of expression through RNA secondary structure, will also be attempted and implemented in a high-throughput condition search robot. Both microbial and eukaryotic systems will be the sources for target selection. Our initial prokaryotic targets will be from Caulobacter crescentus, a microbial system whose asymmetric division offers a model system for cell polarity and that has promise as a bioremediation agent. Drosophila melanogaster will be the eukaryotic organism that we will initially study. Drosophila has been well characterized in particular in regard to developmental pathways. Both organisms have sequenced genomes and are well studied by a variety of genetic and biochemical methods, thus providing a basis for biological and cellular understanding of the roles these proteins and complexes mediate. Human protein complexes, identified from other organisms such as yeast and Drosophila, will ultimately be targets for this project as well.

\section{Accomplishments}

During the past year we completed the installation and upgrade of the TECAN Genesis 200 liquid-handling robot that serves as a critical instrument in several steps in the screening for expression, solubility, and purification of multi-protein complexes. This instrument is currently being utilized in expression and solubility screening tests. We also have been able to isolate podJ, a target from Caulobacter that functions in protein localization for the assembly of polar pili. This protein is being tagged to enable the purification of stable complexes during different points in the cell cycle. Other targets have been selected that are well-studied by genetic, microarray, and imaging methods. 


\section{Project Title. "Structural Genomics Tools for Membrane Proteins" PI(s). Kim, Sung-Hou; Berry, Ed; Downing, Ken; Glaeser, Robert}

\section{Project Description}

The goal of this highly collaborative LDRD project was to develop new approaches for over-expression, protein production and stabilization, and crystallographic structure analysis of membrane proteins.

\section{Accomplishments}

I. Chemotaxis receptors: We have cloned 20 prokaryotic chemotaxis receptors or presumed receptors with an $\mathrm{N}$-terminal $\mathrm{His}_{6}$ tag, a C-terminal $\mathrm{His}_{6}$ tag or double $\mathrm{N}$-and C-terminal $\mathrm{His}_{6}$ tag. Expression of these clones was tested in an E. coli host lacking chemotaxis receptors, HCB721(DE3). Twelve clones expressed at levels suitable for scale-up and purification. Of these twelve, we have purified the chemotaxis receptors from two clones (EC90 and BS82) in large quantities for preliminary crystallization studies. EC90 is the receptor for ribose/galactose and the ligand for BS82 is not known. We have screened several different ways of opening the cells, several different detergents to solubilize the membrane fraction, and several different conditions and columns to purify them. From these multiple screenings, we were able to find the "optimum" process for purification for each. Using the purified proteins we have screened $96 \times 4$ different crystallization conditions at two temperatures. From these screens we found preliminary crystals from each protein. Both crystals at present are small, and the EC90 crystals, which we have several, diffract only to about $20 \AA$ resolution using one freezing condition. The preliminary diffraction studies of several different EC90 crystals show crystal polymorphism as suggested by several space groups and cell parameters. These are, however, not independent, but approximately interconvertible.

II. Phasing Algorithm: In Project I we demonstrated that the phasing algorithm intended for use with electron diffraction data obtained from 2-D crystals, demonstrated previously to work well with synthetic data, requires information about surface undulations as well as the thickness of the minimum, flat slab that contains the 2$\mathrm{D}$ crystal. Still to be determined is whether iterative shrink-wrapping of the compact support is powerful enough to provide the required surface undulations.

III. Membrane protei stability: In Project II we successfully used intrinsic protein fluorescence spectroscopy to demonstrate that membrane proteins are, indeed, more stable in hydrated phosphatidylethanolamine (PE) "cubic phase" gels than they are in hydrated monoglyceride gels. We also successfully demonstrated that fusogenic lipids could be used in low amounts to "catalyze" the facile hydration and formation of such gels under conditions that were not harmful for the membrane proteins that we wished to crystallize. High-throughput methodology was then developed for doing crystallizations and screening in a 96-well format. 
Project Title: Allosteric Mechanisms in Proteins Involved in Cell Signaling Principal Investigator: Kuriyan, John

Project Description:

This project seeks to apply NMR spectroscopy to understand the nature of protein dynamics and conformational changes in protein kinases and other proteins involved in cell signaling. Signaling proteins switch between on and off states during the normal course of their functioning, and the stability and rates of interconversion between these states are modulated by other proteins, small molecules, or covalent modifications. These allosteric effectors alter the dynamics of the protein, but these phenomena are as yet poorly understood in detail.

NMR spectroscopy provides the ultimate experimental probe of protein dynamics, and enables in principle a description of the extent and timescales of molecular motion with information localized to particular residues. Unfortunately, protein kinases present challenging problems for NMR experiments. The kinases that we are interested in have been difficult to express in large yields in bacteria, particularly when using the minimal media that is a requirement for isoptopic labeling of proteins. Even when successfully expressed, the proteins have been unstable and difficult to maintain in the NRM sample tube. Finally, the efficient switching of the purified proteins from one homogeneous state to another has been difficult to accomplish in the test tube. The general aim of the LDRD project has been to overcome these technical hurdles for three classes of protein kinases: the p21-activated kinases (PAK kinases), the Src family of tyrosine kinases and the Abelson Leukemia virus tyrosine kinase $(\mathrm{Abl})$.

The practical importance of this project is illustrated by the cancer drug Gleevec, which selects between very similar protein kinase targets with high specificity. Our previous work has demonstrated that this specificity arises from the ability of the drug to select particular conformations of its target proteins. This knowledge has motivated our interest in understanding the nature of the conformational changes in protein kinases.

Accomplishments:

Building on work done in years 1 and 2 of the project, when successful high yield bacterial expression systems were developed for kinases in all three categories, the previous year has seen substantial advances in the actual NMR spectra that have been obtained. The most progress has been obtained for the PAK kinase, for which conditions for obtaining high quality NMR spectra in the on (phosphorylated) and off (unphosphorylated) states have now been defined, with deuteration of the protein to enhance the signal. Reasonable quality NMR spectra are also now being obtained for the Src and Abl kinases. These studies are being augmented by molecular dynamics simulations on each of the proteins to provide some insight into the nature of the molecular motions as a function of phosphorylation and activation state. 
Project Title: Structure and Functional Characterization of Heme Protein Sensors Principal Investigator: Michael A. Marletta

Project Description: The overall goal of this project is to develop a molecular logic for the design of heme protein sensors. Through our continued characterization of the nitric oxide (NO) sensor from the mammalian soluble guanylate cyclase (sGC) enzyme and the putative NO sensor sGC's in C. elegans, we have found a family of heme-protein sensors in prokaryotes. In facultative aerobes, these predicted proteins were present in histidine kinase-containing operons, suggestive of a two-component-like signaling system. In obligate anaerobes, the predicted heme sensor is fused to a methyl-accepting chemotaxis domain and binds $\mathrm{O}_{2}$, suggestive that the protein is serving as an $\mathrm{O}_{2}$ sensor in a chemotaxis response. The overall questions include: 1) the function of these proteins, 2) the biological ligands that control the activity, and 3) structural determinates for ligand specificity. We have named this family of proteins H-NOX domains (Heme-Nitric oxide OXygen binding domains) to fully encompass their ligand binding properties. The proposal will involve the following specific aims: (i) continue to explore the nature of these predicted cyclases from C. elegans and to determine the function of these proteins, (ii) clone, isolate and characterize H-NOX domains from Caulobacter crescentus, Vibrio cholerae, Shewanella oneidensis and Thermoanaerobacter tengcogensis, (iii) to pursue crystal structures for all constructs that crystallize.

Accomplishments: We obtained the first structure of an H-NOX domain. The H-NOX is from T. tengcogensis and we hypothesize that it is a sensor for $\mathrm{O}_{2}$. The structure, obtained in collaboration with John Kuriyan, was published this past year (Pellicena et al. 2004). It is the first of the sGC-related heme domain structures. We have been able to discern from the structure a molecular explanation for ligand discrimation and further biochemical experiments support our initial conclusions from the structure. We have cloned and continue to characterize H-NOX domains from other prokaryotic sources. VCA0720 from Vibrio cholerae and, as mentioned above, $T t$ Tar4H from $T$. tengcogensis are hemoproteins that are related to the heme domain from sGC and are able to discriminate between $\mathrm{NO}$ and $\mathrm{O}_{2}$. Our further characterization of these proteins was reported this past year (Karow et al. 2004). Briefly, for VCA0720 the ferrous unligated form of VCA0720 is 5-coordinate, high-spin. The CO complex is low-spin, 6coordinate and the NO complex is high-spin and 5-coordinate. These ligand-binding properties are very similar to sGC. TtTar4H forms a low-spin, 6-coordinate ferrous-oxy complex, the first of this sGC-related family that binds $\mathrm{O}_{2}$. TtTar4H has ligand binding properties similar to hemecontaining, $\mathrm{O}_{2}$-sensors. Lastly we have made a remarkable discovery regarding the putative sGC's in C. elegans, also published this past year (Gray et al. 2004). We showed that the $C$. elegans exhibits a strong behavioral preference for 5-12\% oxygen in oxygen gradients, avoiding higher and lower levels. Many C. elegans sensory neurons use cGMP in sensory transduction; avoidance of high oxygen requires the soluble guanylate cyclase homolog gcy-35 and the cGMPgated channel tax-2/tax-4, implicating cGMP in oxygen sensation. The GCY-35 heme domain binds molecular oxygen, unlike the heme domains of classical NO-regulated guanylate cyclases. GCY-35 and TAX-4 mediate oxygen sensation in URX, AQR, and PQR sensory neurons, which control a naturally polymorphic social feeding behavior in $C$. elegans. Social feeding and related behaviors occur only when oxygen exceeds C. elegans' preferred level, and require gcy-35 activity. Our results suggest that GCY-35 is regulated by molecular oxygen, and that social feeding can be a behavioral strategy for responding to hyperoxic environments. 
Development of techniques for the study of large macromolecular complexes using X-ray crystallography

Co-Principle Investigators: Gerry McDermott and Robert Tjian

Project Description

As our understanding of biological mechanisms becomes more sophisticated, it has become evident that such structural studies are necessary to understand dynamically regulated processes such as DNA replication, transcription, RNA processing, and translation - all of which require the coordinated action of very large multi-subunit enzymes and protein complexes. While our previous studies have focused on low resolution structures of various transcription complexes using electron microscopy, it has become apparent that higher resolution structures are necessary to understand the detailed mechanisms of transcriptional regulation. The purpose of this research is to implement novel strategies for crystallographic analysis of large macromolecular assemblies.

To date, it has been extremely difficult to over-express and assemble large multi-subunit complexes in quantities sufficient for crystallographic analysis, consequently this work will largely depend on endogenous complexes isolated directly from cells. First, we will be initiating the large scale immunopurification of human transcription complexes from HeLa cells. Second, we will be biochemically characterizing the complexes to obtain information on the heterogeneity and subunit organization of these large macromolecular assemblies. Purification protocols will be modified based on our biochemical studies to obtain samples that are both structurally and functionally homogenous. Finally, we will be using a combination of robotic and microfluidic devices to crystallographically screen nanoliter amounts of our endogenously purified protein complexes. Once potential crystallization conditions are obtained we will scale up the crystallization conditions and assess the quality of the crystals using the ALS synchrotron.

\section{Accomplishments}

We have recently succeeded in optimizing the large scale purification of the human TFIID(1MDa) and the RNA polymerase II(0.7MDa) complexes from HeLa cells. Using a combination of techniques including SDS-PAGE, mass spectrometry, and gel filtration, we are currently assessing the macroheterogeneity and microheterogeneity resulting from the substoichiometric association and post-translational modifications of subunits. In addition, we are using chemical crosslinking and functional interaction assays to address the subunit organization of these complexes along with potential conformational changes within these complexes. Interestingly, we see a number of conformational changes occurring within the TFIID complex upon addition of various transcription factors. We are currently trying to correlate these structural changes with functional steps in transcription pre-initiation complex formation.

In addition, we are trying to determine conditions(salts, detergents, etc.) that will lock TFIID into a single conformation for crystallization trials. Once we are confident in the homogeneity of our complexes, we will subject our TFIID and RNA polymerase II complexes to nanoliter crystallization trials. Initial crystallization of test proteins at the nanoliter scale has been achieved, indicating the feasibility of our approach. 
Conformation and Reaction Dynamics at the Single Molecule Level Principal Investigator: Haw Yang

\section{Project Description:}

This research project is focused on developing single-molecule spectroscopic techniques for advancements in the design and application of single-molecule assays. The emerging picture of inferring biomolecule functionality from its 3-dimensioanl structure is incomplete without a fundamental understanding of the microscopic dynamics. The capability of single-molecule spectroscopy offers a unique means to bridge the gap between the static structure and the dynamic functionality. The successful conclusion of this project will complement other ongoing research efforts at LBNL including structural biology, microscopy, and biological dynamics, to influence positively the thinking in the post-genome era. In accordance with the principles of the LDRD program, the proposed project will progress through a number of pilot experiments aimed at technological advancement. The goal of the FY04 proposal is to continue advancing these ideas and to apply the new concepts developed during FY03 to build advanced single-molecule microscopes (in collaboration with UCB Chemistry).

Accomplishments:

Time-resolved single molecule fluorescence measurements may be used to probe the conformational dynamics of biological macromolecules. The best time resolution in such techniques will only be achieved by measuring the arrival times of individual photons at the detector. New analysis methods are needed to take advantage of this capability.

Adenylate kinase (AKe) from $E$. coli maintains the energy balance in a cell and serves as a prototypical system for detailed dynamic studies of structure-function relationship. We have used the newly constructed, server-based single-molecule microscope to examine the conformation dynamics of individual Ake. Experimental data was analyzed photon by photon using the maximum-information and the change-point methods. In the absence of substrates, it was found that AKe exists in two distinct conformations. Potential of mean force corresponding to the said distribution was also deduced from experimental data. The conformational motions was modeled as a fictitious particle moving along the potential energy surface following the Lengevin dynamics. 


\title{
Physics Division
}

LB03033

\author{
Future Experiments in Neutrino Physics \\ Principal Investigators: Stuart Freedman and Jesse Goldman
}

Project Description

Our understanding of the neutrino world has changed dramatically in the past two years. Recent experiments have confirmed earlier evidence that the three types of neutrinos mix; that is, they change into one another. According to the predictions from the Standard Model, neutrinos/antineutrinos are without mass. Contrary to this, over the past two years, solar neutrino experiments at the SNO and Super-K detectors implied that the ghostlike snippets of matter/antimatter do possess enough mass to enable them to oscillate and change flavor over a distance. Kamioka Liquid Scintillator Anti-Neutrino Detector (KamLAND) is the first experiment to observe the neutrino properties responsible for solar neutrino flavor changes from a terrestrial source, the reactors in Japan's nuclear power plants.

We propose to carry out a series of studies to investigate in detail the properties of the neutrinos and define the next generation experiments to better understand the neutrino sector. The proposed work will focus on three promising areas that build on Berkeley Lab's work in KamLAND and underground at Kamioka making use of special experience of postdoc fellow, Jesse Goldman.

- A solar program with KamLAND is not presently funded in either Japan or the U.S. We will help in understanding the technical issues related to a solar 7Be experiment with KamLAND. This experiment should provide better constraints on $\sin 2(2 \mathrm{e})$ than is possible with reactor experiments.

- KamLAND, located in a mine cavern beneath the mountains of Japan's main island of Honshu near the city of Toyama, is the largest low-energy anti-neutrino detector ever built. Data obtained with this detector indicates that the scintillator has very low contamination of uranium, thorium, and potassium. The detector should be capable of detecting low energy solar neutrinos if the levels of radioactive krypton and lead are reduced. The case for a solar phase of the experiment will be based on the feasibility of this purification process.

- We will participate in a study of possible opportunities for small underground experiments at Kamioka or other laboratories. Under present consideration is a measurement of positronium decaying to nothing: a test of theories with extra dimensions. This rather modest experiment should be carried out underground to avoid cosmic ray backgrounds.

\section{Accomplishments}

The work in FY04 was focused on the technical issues related of measuring neutrino mixing at reactors. During the year, Jesse Goldman adapted KamLAND data and Monte Carlo simulation to study issues that would relate to a new experiment to measure the last mixing angle theta 13 at reactors. This work included issues related to calibration of such a detector using contaminating radio-nuclei such as $12 \mathrm{~B}$ and $9 \mathrm{Li}$. His analysis included determinations of residual contaminates that would affect the new experiment. He also contributed to work on the Monte Carlo simulation now being adapted to simulate the new reactor experiment. 


\author{
Designing a Novel Reactor Neutrino Oscillation Experiment \\ for Measuring the Unknown Mixing Angle $\theta_{13}$ \\ Principal Investigators: S.J. Freedman, K.M. Heeger, R.W. Kadel, K.-B. Luk
}

\title{
Project Description
}

The purpose of this project is to develop a design concept and research detector technology for a next-generation reactor neutrino oscillation experiment. Using multiple detectors placed at different distances from a nuclear power plant a reactor antineutrino experiment can search for subdominant oscillation effects in the $v_{\mathrm{e}} \rightarrow v_{\mu, \tau}$ channel and make a precision measurement of the unknown neutrino mixing angle $\theta_{13}$. The discovery of subdominant oscillation and non-zero $\theta_{13}$ would have a profound impact in neutrino physics. The size of $\theta_{13}$ is critical for $\mathrm{CP}$ violation searches in the lepton sector and will define research in neutrino oscillation physics for the next decade and beyond. Neutrino oscillation and CP violation in the lepton sector may lead to leptogenesis and ultimately may explain the observed baryon asymmetry in the Universe.

Our primary goals are to select a suitable experimental site, perform design and engineering studies for future neutrino detectors, prototype detector components, and assess possible backgrounds to this precision neutrino measurement through simulation and environmental measurements. Mechanical and civil engineering studies are needed to demonstrate the technical feasibility of the experiment. An international scientific collaboration will be formed to conduct this challenging experiment. Domestic and overseas locations for the experiment are under consideration and will be evaluated in the light of their scientific potential and the expeditious timescale envisioned for this experiment.

\section{Accomplishments}

In the past year we developed a conceptual design for an underground neutrino experiment at the Diablo Canyon site in California or the Daya Bay nuclear power plant in China, the two best sites worldwide for conducting a reactor $\theta_{13}$ experiment with horizontal access tunnels. Mechanical engineering and civil construction studies were performed including an evaluation of means and methods for the construction of the underground detector halls. A geological investigation of the Diablo Canyon site has been completed and is underway at Daya Bay in China. A preliminary cost estimate to establish the technical scope and feasibility of the project was prepared and reviewed. We performed detector simulations in support of engineering studies to characterize the physics potential and the ultimate sensitivity of the experiment. The timely realization of this experiment are critical to its success, and we have started parallel efforts on the design and engineering as well as the physics studies for this project.

Scientists from Berkeley have played a lead role in establishing the case for a future reactor neutrino project in the international scientific community and in early 2004 Lawrence Berkeley National Laboratory formed a R\&D collaboration with the Institute of High Energy Physics (IHEP) in Beijing for the development of this experiment. Recently, the APS Neutrino Study recommended a reactor $\theta_{13}$ project as a high priority for the US program in neutrino physics. Together with our collaborators we are now investigating the possibility of a timely and cost-effective realization of this project at the Daya Bay nuclear power plant in China. 


\section{Cross-Divisional}

LB03035

Microscopic Theory of Protein Surface

Principal Investigator: Phillip Geissler

\section{Project Description}

This project aims to develop a quantitative understanding of mechanics and fluctuations at the molecular surfaces of proteins. Of particular interest is the way such surfaces have been designed through evolution to facilitate molecular recognition and binding and to control association into larger assemblies. We will develop and analyze models at several levels of detail to reveal the statistical mechanics of conformational changes on the protein exterior. Results of this analysis will aid in systematic redesign of a protein's composition to modify its interactions with ligands, other proteins, and solvent.

We will examine the degree to which steric constraints on the atomic scale couple motions near the surface to larger reorganization within a molecule's interior. For this purpose we will explore possible repackings of amino acid side chains near a protein's native state as a measure of flexibility and correlations among local rearrangements. With more detailed energetic models we will investigate examples of small molecule binding events that generate large-scale structural changes, such as in certain transmembrane ion channels. In this case we will focus on the transmission of stress from the binding pocket to distant regions of molecules and complexes. Finally, using schematic models, we will study the relationship between spatial distributions of structural fluctuations and the evolution of protein composition.

\section{Accomplishments}

Progress had been made over the past year in each component of this project. We have developed a computationally efficient algorithm for sampling sterically allowed side chain arrangements in large molecules. This method combines several Monte Carlo approaches, such as umbrella sampling and thermodynamic integration, to estimate the total number of rearranged structures consistent with geometrical constraints. We are now applying it to a specific protein whose side chain mobilities are suggested by experiments to have unexplained long-range correlations.

Atomistic calculations have been initiated to probe physically plausiable mechanisms for ligand-driven ion channel gating. These calculations will harvest examples of reversible gating events. From such trajectories we will study how stress develops and propagates within and among molecules comprising the channel.

Finally, we have simulated the evolution of a schematic model protein. Random mutations were introduced with the constraint that folding to the native state must remain efficient. Our eventual goal is to determine how constraints of function (related to surface properties) modify this basic evolution, but we have found several unexpected subtleties in the way constraints on folding alone shape mutability in a protein's sequence. We are currently studying genomic data to verify model results. 


\section{Investigation of charge transfer in organic electronics using ultrafast spectroscopy and targeted synthesis \\ PIs: Harris/Arnold(CSD); Kerr/Johnson(EETD)}

Project Description: The purpose of the proposal is to develop an understanding of the dynamics and mechanisms of charge transfer at interfaces in organic electronic devices. Organic light emitting diodes (OLED), photovoltaic cells, and thin film transistors (TFTs) require fundamental understanding of the interfacial charge injection and transport processes to realize their potential. We focus a unique combination of capabilities on this critical area. The three major components of the research are:

1. Application of ultrafast (femtosecond) spectroscopy techniques to organic-cathode interfaces for small molecule and polymer LEDs and to organic photovoltaic systems that utilize fullerenes, photo-sensitizers and conducting polymers.

2. Synthesis of appropriate new molecules to facilitate the interfacial analyses and to provide relevant structure-function information.

3. Construction and testing of devices that incorporate the new interfacial structures based on designs derived from the spectral analysis and structure-function relationships.

Synthesis and Devices: We have synthesized a variety of new molecules, including 9,9-dimethylfluorene monomers and dimers, 9,9-dihexylfluorene monomers, and 9,9-dipropylfluorene monomers. These molecules have allowed us to study trends in the electronic behavior of interfacial electrons as a function of the electronic structure (e.g. the difference in conjugation length between monomers and dimers) as well as side chain substitution (from methyl through hexyl side chains). We have optimized our techniques for building organic light emitting diodes of blue emitting polyfluorenes, which allows us to compare the performance of real devices with the $2 \mathrm{PPE}$ spectroscopic measurements. These devices perform comparably with state of the art devices, and studies of their physical and chemical degradation pathways are underway.

\section{Ultrafast Spectroscopy:}

$C_{60} / A g(111)$ : We have mapped the occupied and unoccupied 3D band structure as a function of film growth (from 1-10 monolayers). From the effective mass, $m^{*}$, of the molecular states, we can estimate the transfer integral, $\beta$, as well as the symmetry of the molecular states that form the bands.

Fluorenes/Ag(111): We have directly measured the energy of the LUMO with respect to the Fermi level, $E_{F}$, in monomer and dimer films; this parameter is critical to charge injection in devices. Experiments are underway to measure the trend in the energy of the LUMO as a function of conjugation length and side chain functionality.

PTCDA/Ag(111): We have studied the effects of film morphology on the band structure and dynamics of interfacial electrons and excitons in films from 1-5 monolayers thick. Using a theory developed in this lab, we can estimate the size of the interfacial Frenkel excitons.

Intraband relaxation: We have developed a theoretical formalism that describes momentum relaxation as a stochastic process. The extracted diffusion constants for the interfacial electrons are remarkably similar to the diffusion of a conduction electron in the bulk substrate. This suggests that the intraband relaxation is dominated by scattering of the surface charge (image charge) in the substrate. This energy loss near the surface may affect recombination currents near device electrodes as well as power consumption. 
"Ex-Situ" and "Remote" Molecular Imaging and Spectroscopy

Principal Investigators: Alex Pines, John Clarke, David Wemmer, Jeff Reimer, Steven Gourlay, Gian Carlo Sabbi, Ernest Majer, Dimitris Budker and Thomas Budinger

\section{Project Description}

Imaging and spectroscopic analysis by magnetic resonance (NMR/MRI) are typically performed by immersing the sample, object or subject into a radiofrequency coil within the bore of a high-field superconducting magnet, thereby limiting the systems and environments for which they may be used. The work conducted under the LDRD sought to develop a new interdisciplinary program to take NMR and MRI into exciting new territory, by implementing novel, seemingly impossible, methods and devices of "ex-situ" and "remote" detection, with applications over distance scales from nanometers to meters. This will allow enhanced, noninvasive, even surreptitious, scanning detection, ranging over the investigation of materials and surfaces, porous systems relevant to earth sciences, well-logging, fluid flow, multiplexed screening and assays of target molecules, functional molecular imaging of metabolites and brain function, and an approach to objects and subjects relevant to counterterrorism.

\section{Accomplishments}

Significant progress was made to develop and implement several of the goals for the project.

Construction and installation of a new generation of one-sided ex-situ magnet as well as a device that allows for the rotation of the magnetic field was completed and initial experiments conducted. New superconducting magnets possessing external tunable "sweet spots" for insideout standoff NMR/MRI will make possible a truly portable incarnation of our high resolution NMR in inhomogenous fields outside the radiofrequency coil.

In the area of hand-held magnetic spectroscopy, imaging and elastrography, our efforts concentrated on imaging and acquiring chemical shift information in a simulated ex situ environment. A set of proof-of-principle imaging and spectroscopy experiments were performed inside a superconducting magnet while an imaging gradient along one dimension, $x$, was constantly applied to simulate an inherent inhomogeneity and while the $y$ and $z$ gradients were controlled. A conical radiofrequency (rf) coil geometry was employed to create an inhomogeneous $\mathrm{rf}$ field along the same direction. These experiments yielded satisfactory results.

Work continued on the development and implementation of magnetic resonance theory, multidimensional pulse sequences, and consoles for high-resolution ex-situ images and spectra in the presence of field gradients and rotation. With small powerful scanning magnets, images and spectra will be resolvable at distances up to centimeters in the initial magnet/spectrometer designs and sequences. Micro- and nanoscale detection of surfaces is necessarily ex-situ and occurs with inhomogeneous magnetic fields. The ex-situ methodology will be scaled down so as to investigate the possibility of producing resolved images and spectra of micro- and nanosystems with high-sensitivity optical detection.

A number of areas of specific applications were also studied. For gas and oil exploration, the first remotely detected 2D Xe-129 NMR image of channel pores ( $1 \mathrm{~mm}$ inner diameter) was obtained. The characterization of xenon binding sites into proteins that change affinity and/or shift in CheY has been completed. One research area to date has pursued the development and implementation of a new generation of room-temperature access mobile SQUID detectors for low-frequency flux detection of samples both ex-situ and for remote reconstruction of images and spectra using thermally prepolarized systems encoded laser-polarized gases. Finally was the completion of the Magic-Angle Rotating Field (BoMAS) Spectrometer: 


\section{Publications List}

AFRD-Dietderich LB03009 Short Period Superconducting Undulator Development

R. D. Schlueter et al., Synchrotron Radiation News, vol. 17, No. 1, Jan/Feb. 2004

S. Prestemon et al, "Design, Fabrication, and Test Results of Undulators Using $\mathrm{Nb}_{3} \mathrm{Sn}$

Superconductor", Presented at ASC2004, Jacksonville, F1. Oct. 3-8, 2004. To be published in IEEE Transactions on Applied Superconductivity, June 2005.

AFRD-Fawley LB04040 Analysis and Modeling of Multicore Induction Cell Voltage Distribution

Richard J. Briggs and William M. Fawley, "A Simple Model for Induction Core Voltage Distributions"; LBNL-55079; also catalogued at LANL as DARHT Technical Note No. 418, 15 July 2004.

Richard Briggs, William Fawley, Lou Reginato, and Will Waldron, "One-Core, RadiallyResolved Voltage Measurements at the LBNL Induction Core Test Stand "; draft to be issued as LBNL report.

AFRD-Leemans LB02001 Novel Coherent THz and IR Source Using a Laser Wakefield Accelerator and Applications

J. Van Tilborg, C.B. Schroeder, E. Esarey and W.P. Leemans, "Pulse shape and spectrum of coherent diffraction-limited transition radiation from electron beams", Lasers and Particle Beams, to be published.

J. van Tilborg, C. G. R. Geddes, C. Tóth, E. Esarey, C. B. Schroeder, M. C. Martin, Z. Hao, and W. P. Leemans, "Coherent Transition Radiation From a Laser Wakefield Accelerator as an Electron Bunch Diagnostic", Proceedings of the Advanced Accelerator Workshop, Stony Brook, June 21-26, 2004, to be published.

J. Faure, J. Van Tilborg, R.A. Kaindl, W.P. Leemans, "Modelling laser-based table-top THz sources: Optical rectification, propagation and electro-optic sampling" [Review]. Optical \& Quantum Electronics 36(8):681-697, June 2004.

W.P. Leemans, J. van Tilborg, J. Faure, C.G.R. Geddes, C. Toth, C. B. Schroeder, E. Esarey, G. Fubiani, G. Dugan, "Terahertz radiation from laser accelerated electron bunches" [Article]. Physics of Plasmas 11(5):2899-2906, May 2004.

C.B. Schroeder, E. Esarey, J. van Tilborg, W.P. Leemans, "Theory of coherent transition radiation generated at a plasma-vacuum interface" - article no. 016501. Physical Review E 6901(1 Part 2):6501, January 2004.

AFRD-Lidia LB04019 Critical Accelerator Technologies for Future Advanced Light Sources

A. Zholents, W. Fawley, "Proposal for Intense Attosecond Radiation from an X-ray FreeElectron Laser," Phys. Rev. Lett. 92, 224801, 4 June 2004.

J. Corlett, W. Barletta, et. al., "LUX - A Design Study for a Linac/Laser-Based Ultrafast Xray Source," Proc. Fourth Generation X-Ray Sources and Optics II, SPIE Proc. Vol. 5534, August 2004.

A. Zholents, W. Fawley, "Toward Attosecond X-ray Pulses from the FEL Proc. Fourth Generation X-Ray Sources and Optics II," SPIE Proc. Vol. 5534, August 2004.

J. Corlett, W Fawley et. al., "Harmonic Cascade FEL Designs for LUX, a Facility for Ultrafast X-ray Science," Proc. $26^{\text {th }}$ International Free Electron Laser Conference, August 2004. 
A. Zholents, W. Fawley et. al., Current-Enhanced SASE Using an Optical Laser and its Application to the LCLS," Proc. $26^{\text {th }}$ International Free Electron Laser Conference, August 2004.

G. Penn, M. Reinsch, J. Wurtele, "Analytic Model of Harmonic Generation in the Low-Gain FEL Regime," Proc. $26^{\text {th }}$ International Free Electron Laser Conference, August 2004

A. Zholents, "Proposal for Current-Enhanced SASE X-ray FEL," LBID-2497, February 2004.

J. Corlett, W. Barletta et. al., "LUX - A Recirculating Linac-Based Facility for Ultrafast Xray Science," Proc. Ninth European Particle Accelerator Conference, July 2004.

A. Zholents, W. Fawley, "Towards Attosecond X-Ray Pulses from the FEL," Proc. Ninth European Particle Accelerator Conference, ” July 2004.

J. Staples, S.Virostek, S. Lidia, "Engineering Design of the LUX Photoinjector," Proc. Ninth European Particle Accelerator Conference, July 2004.

R. Wilcox, J. Staples, L. Doolittle, “A Fiber Optic Synchronization System for LUX," Proc. Ninth European Particle Accelerator Conference, July 2004.

S. Lidia, "Design of Injector Systems for LUX," Proc. Ninth European Particle Accelerator Conference, July 2004.

W. Wan, J. Corlett, W. Fawley, A. Zholents, "Design Study of the Bending Sections between Harmonic Cascade FEL Stages," Proc. Ninth European Particle Accelerator Conference, July 2004.

G. Stover, "A Kicker Design for the Rapid Transfer of the Electron Beam Between Radiator Beamlines in LUX," Proc. Ninth European Particle Accelerator Conference, July 2004.

G. Penn, M. Reinsch et. al., Harmonic Cascade FEL Designs for LUX," Proc. Ninth European Particle Accelerator Conference, July 2004.

F. Banfi et. al., "Photoemission Processes in Metals and Other Photocathodes Materials in the Femtosecond Regime," LUX Tech Note-019, CBP Note-638, September 2004.

S. Lidia, "Emittance Compensation of Intense, Magnetized Beams and Optimization of Photoinjectors for Future Light Sources," LBNL-56558 in preparation.

Y.-E. Sun, S. Lidia et. al., "Generation of Angular-Momentum-Dominated Electron Beams from a Photoinjector," submitted for publication to Phys. Rev. ST Accel. Beams, November 2004.

AFRD-Ryne LB03021 Optimal Solvers for Infinite-Dimensional Hamiltonian Systems

I.V.Pogorelov, J.Qiang, R.D.Ryne, and R.Gluckstern, "Symplectic Discretization of infinitedimensional Hamiltonian systems" in preparation for submission to Journal of Computational Physics.

I.V.Pogorelov and B.Terzic, "Wavelet-based Poisson solver for beam dynamics simulations" in preparation for submission to SIAM Journal on Scientific Computing. Preliminary version to appear in Conference Proceedings of the Henry Kandrup Memorial Workshop in Nonlinear Dynamics (2004, Gainesville, FL).

AFRD-Sabbi LB03022 Superconducting Magnet Systems for Ex-situ NMR Spectroscopy

G. Sabbi, Specifications, Design Issues and Features of the Ex-Situ NMR Superconducting Magnet Prototype," unpublished internal note (September 22, 2004) pending patent application. 
AFRD-Schenkel LB02002 Solid State Quantum Computer Development with Single Ion Implantation

T. Schenkel, I. W. Rangelow, R. Keller, S. J. Park, J. Nilsson, A. Persaud, V. R. Radmilovic, P. Grabiec, D. H. Schneider, J. A. Liddle, and J. Bokor, "Open questions in electronic sputtering of solids by slow highly charged ions with respect to applications in single ion implantation," Nucl. Instr. Meth. B 219-220, 200 (2004).

S.-J. Park, A. Persaud, J. A. Liddle, J. Nilsson, J. Bokor, D. H. Schneider, I. Rangelow and T. Schenkel, "Processing Issues in Top-Down Approaches to Quantum Computer Development in Silicon," Microelectronic Engineering 73-74, 695 (2004).

A. Persaud, S. J. Park, J. A. Liddle, I. W. Rangelow, J. Bokor, R. Keller, F. I. Allen, and D. H. Schneider, "Quantum Computer Development with Single Ion Implantation," Quantum Information Processing 3, 233, (2004).

S. J. Park, A. Liddle, A. Persaud, F. I. Allen, T. Schenkel, "Formation of $15 \mathrm{~nm}$ scale Coulomb blockade structures in silicon by Electron Beam Lithography with a bi-layer resist process," J. Vac. Sci. Technol. B, Nov./Dec. issue (2004).

A. Persaud, F. I. Allen, F. Gicquel, S. J. Park, J. A. Liddle, J. Bokor, Tzv. Ivanov, K. Ivanova, I. W. Rangelow, and T. Schenkel, "Single Ion Implantation with Scanning Probe Alignment," J. Vac. Sci. Technol. B, Nov./Dec. issue (2004).

AFRD-Vay/Furman LB03028 Electron Production and Collective Field Generation in Intense Particle Beams

R. H. Cohen, A. Azevedo, A. Friedman, M. A. Furman, S. M. Lund, A. W. Molvik, P. H. Stoltz, J.-L. Vay, S. Veitzer, "Modeling Electron Cloud Effects in Heavy-Ion Accelerators," Proc. 31st ICFA Advanced Beam Dynamics Workshop on Electron-Cloud Effects "ECLOUD04", Napa (California), April 19-23, 2004 (invited paper). Url: http://mafurman.lbl.gov/ECLOUD04 proceedings/cohen_ms-ECLOUD04-v3.pdf

A. W. Molvik, F. M. Bieniosek, R. H. Cohen, S. Eylon, A. Faltens, A. Friedman, E. Henestroza, M. Kireeff-Covo, J. W. Kwan, S. M. Lund, L. Prost, P. K. Roy, P. A. SeidL, J.-L. Vay, G. Westenskow, S. Yu, "Experimental studies of electron and gas sources in a heavy-ion beam," Proc. 31st ICFA Advanced Beam Dynamics Workshop on ElectronCloud Effects "ECLOUD04", Napa (California), April 19-23, 2004 (invited paper). Url: http://mafurman.lbl.gov/ECLOUD04 proceedings/Molvik_ECloud04_JACoW-v2.pdf

A. W. Molvik, M. Kireeff-Covo, F. M. Bieniosek, L. Prost, P. A. Seidl, D. Baca, A. Coorey, and A. Sakumi, "Gas desorption and electron emission from $1 \mathrm{MeV}$ potassium ion bombardment of stainless steel," submitted to Physical Review Special Topics Accelerators and Beams, April 2004. Url: http://prstab.aps.org/pdf/PRSTAB/v7/i9/e093202

Peter Stoltz, Seth Veitzer, Ron Cohen, Art Molvik, Miguel Furman, Jean-Luc Vay, “The CMEE Library for Numerical Modeling of Electron Effects", Proc. 31st ICFA Advanced Beam Dynamics Workshop on Electron-Cloud Effects "ECLOUD04," Napa (California), April 19-23, 2004. Url: http://mafurman.lbl.gov/ECLOUD04 proceedings/stoltz ecloud04-v2.pdf

J.-L. Vay, M. A. Furman, A. W. Azevedo, R. H. Cohen, A. Friedman, D. P. Grote, P. H. Stoltz, S. A. Veitzer, "Status report on the merging of electron-cloud code POSINST with 3-D accelerator PIC code WARP", Proc. 31st ICFA Advanced Beam Dynamics Workshop on Electron-Cloud Effects "ECLOUD04," Napa (California), April 19-23, 2004. Url: http://mafurman.lbl.gov/ECLOUD04_proceedings/vay_il_ECLOUD04_2.pdf 
ALS-Howells LB04013 Coherent X-ray Diffraction Imaging (CXDI)

Beetz, T., M. R. Howells, C. Jacobsen, C.-C. Kao, J. Kirz, E. Lima, T. O. Mentes, H. Miao a, C. Sanchez-Hanke, D. Sayre, D. Shapiro, "Apparatus for X-ray diffraction microscopy and tomography of cryo specimens," Accepted by Nucl. Instrum. Meth. A, (2004).

Carrozini, B., G. Cascarano, L. D. Caro, C. Giacovazzo, S. Marchesini, H. N. Chapman, H. He, M. Howells, J. S. Wu, U. Weierstall, J. C. H. Spence, "Phasing diffuse scattering. Application of the SIR2002 algorithm to the non-crystallographic phase problem," Acta Cryst., A60, 331-338 (2004).

Chapman, H. N., A. Barty, T. Beetz, C. Cui, H. He, M. R. Howells, S. Marchesini, A. Noy, J. C. H. Spence, U. Weierstall, "High-Resolution Three-Dimensional X-ray Diffraction Microscopy," http://electron.lbl.gov/marchesini/pyramid (2004).

Chapman, H. N., S. P. Hau-Riege, R. A. London, S. Marchesini, A. Noy, A. Szoke, H. Szoke, E. Ingerman, J. Hajdu, G. Huldt, M. R. Howells, H. He, J. C. H. Spence, U. Weierstall, "Prospects for single-particle imaging at XFELs," in Proceedings of the LEOS Summer Topical Meetings, 2004, Vol. 04TH8728C, IEEE.

He, H., U. Weierstall, J. C. H. Spence, S. Marchesini, M. Howells, H. A. Padmore, H. Chapman, "Use of extended and prepared reference objects in experimental Fourier transform x-ray holography," Appl. Phys. Lett., 85, 2454-2456 (2004).

Howells, M. R., T. Beetz, H. N. Chapman, C. Cui, J. M. Holton, C. J. Jacobsen, J. Kirz, E. Lima, S. Marchesini, H. Miao, D. Sayre, D. A. Shapiro, J. C. H. Spence, "An assessment of the resolution limitation due to radiation-damage in X-ray diffraction microscopy," submitted to Journal of Electron Spectroscopy and Related Phenomena, (2004).

Shapiro, D., P. Thibault, T. Beetz, V. Elser, M. Howells, C. Jacobsen, J. Kirz, E. Lima, H. Miao, A. Neiman, D. Sayre, "Biological Imaging by Soft X-ray Diffraction Microscopy," submitted to Nature, (2004).

Spence, J. C. H., R. B. Doak, "Single Molecule Diffraction," Phys. Rev. Lett., 92, 198102 (2004).

Spence, J. C. H., K. Schmidt, J. Wu, G. Hembree, U. Weierstall, R. B. Doak, P. Fromme, "Diffraction from a beam of laser-aligned proteins: resolution limits," Submitted to Acta Cryst. A, (2004).

Spence, J. C. H., U. Weierstall, M. R. Howells, "Coherence and sampling requirements in diffractive imaging," Ultramicroscopy, 101, 149-152 (2004).

Thibault, P., "Yeast cell x-ray tomography movie," http://xray1.physics.sunysb.edu/movies/yeast_dec2004.mov (2004).

Marchesini, S., H. N. Chapman, S. P. Hau-Riege, R. A. London, A. Szoke, H. He, M. R. Howells, H. Padmore, R. Rosen, J. C. H. Spence, U. Weierstall, "Coherent X-ray diffractive imaging: applications and limitations," Optics Express, 11, 2344-2353 (2003) http://www.opticsexpress.org/abstract.cfm?URI=OPEX-11-19-2344.

Marchesini, S., H. He, H. N. Chapman, A. Noy, S. P. Hau-Riege, M. R. Howells, U. Weierstall, J. C. H. Spence, "X-ray Image reconstruction from a from a diffraction pattern alone," Phys. Rev. B, 68, 140101(R) (2003).

Spence, J. C. H., M. R. Howells, "Coherent diffractive imaging with x-rays and electrons," in Proceedings of the Eighth International Conference on Synchrotron Radiation Instrumentation, San Francisco, 2003, Vol. CP705, American Institute of Physics. 
ALS-Hussain LB03013 A Novel Ultra-high Resolution $(<10 \mathrm{meV})$ Inelastic Scattering Spectrograph to Study Coupled Electron-Orbiton-Phonon Interactions

Yi-De Chuang, Brooke Mesler et al., "High-Resolution Soft X-Ray Inelastic Scattering Spectrographs at the Advanced Light Source," to be submitted to Journal of Physics and Chemistry of Solids. Also to be published as part of Proceedings of the International Conference on Inelastic Scattering 2004, Argonne.

Yi-De Chuang, D. Qian et al., "Resonant Soft S-Ray Scattering on Half-Doped Bilayer Manganite: Observation of Orbital Ordering Superlattice Reflection," draft to be submitted to Physical Review Letters.

\section{ALS-Kirz LB04044 Lensless Imaging of Yeast Cells}

T. Beetz, M. R. Howells, C. Jacobsen, C.-C. Kao, J. Kirz, E. Lima, T. O. Mentes, H. Miao, C. Sanchez_Hanke, D. Sayre, D. Shapiro; “Apparatus for X-ray diffraction microscopy and tomography of cryo specimens," accepted for publication in Nuclear Instruments and Methods A.

M. R. Howells, T. Beetz, H. N. Chapman, C. Cui, J. M. Holton, C. J. Jacobsen, J. Kirz, E. Lima, S. Marchesini, H. Miao, D. Sayre, D. A. Shapiro, J. C. H. Spence; "An assessment of the resolution limitation due to radiation-damage in X-ray diffraction microscopy, " accepted for publication in Journal of Electron Spectroscopy and Related Phenomena.

ALS-Martin LB02004 Development of a Coherent Far-IR Synchrotron Source

J. M. Byrd, Z. Hao, M. C. Martin, D.S. Robin, F. Sannibale, R.W. Schoenlein, A.A. Zholents, M.S. Zolotorev; "Tailored terahertz pulses from a laser-modulated electron beam," draft to be submitted to Phys. Rev. Lett.

F. Sannibale, J. M. Byrd, Á. Loftsdóttir, M. Venturini, M. Abo-Bakr, J. Feikes, K. Holldack, P. Kuske, G. Wüstefeld, H.-W. Hübers, and R. Warnock, "CSR in storage rings: a model," ICFA (International Committee for Future Accelerators) Beam Dynamics Newsletter, December 2004.

D.N. Basov, J. Feikes, D. Fried, K. Holldack, H.W. Hübers, P. Kuske, M.C. Martin, S. G. Pavlov, U. Schade, E.J. Singley, G. Wüstefeld, "Initial scientific uses of coherent synchrotron radiation in electron storage rings," ICFA (International Committee for Future Accelerators) Beam Dynamics Newsletter, December 2004.

F. Sannibale, J.M. Byrd, Á. Loftsdóttir, M. Venturini, M. Abo-Bakr, J. Feikes, K. Holldack, P. Kuske, and G.Wüstefeld, H.-W. Hübers, and R.Warnock, "A Model Describing Stable Coherent Synchrotron Radiation in Storage Rings," Phys. Rev. Lett. 93094801 (2004). Also selected for the Virtual Journal of Ultrafast Science 3 (9) 2004. LBNL/PUB-890.

J. M. Byrd, S. De Santis, J-Y Jung, D. Li, Michael C. Martin, W. McKinney, D. Munson, H. Nishimura, D. S. Robin, F. Sannibale, R. Schlueter, M. Venturini, W. Wan, M. Zolotorev; "CIRCE, The Coherent Infrared Center at the ALS," European Particle Accelerator Conference, July 2004. LBNL-55603

J.M. Byrd, Z. Hao, M.C. Martin, D.S. Robin, F. Sannibale, R.W. Schoenlein, M. Venturini, A.A. Zholents, M.S. Zolotorev; "Coherent infrared radiation from the als generated via femtosecond laser modulation of the electron beam," European Particle Accelerator Conference, July 2004. LBNL-55688

H. Nishimura, D. Robin, F. Sannibale, W. Wan, "Lattice Studies for CIRCE (Coherent InfraRed CEnter) at the ALS," European Particle Accelerator Conference, July 2004. LBNL-55647 
C. Biscari, J. M. Byrd, M. Castellano, M. Cestelli Guidi, A. Gallo, A. Ghigo, A. Marcelli, F. Marcellini, M.C. Martin, G. Mazzitelli, C. Milardi, P. Morini, M. Piccinini, M. Preger, P. Raimondi, D. Sali, F. Sannibale, M. Serio, M. Zobov; "Terahertz Coherent Synchrotron Radiation at DAFNE," DAFNE Technical Note G-61, July 28, 2004.

E.J. Singley, M. Abo-Bakr, D.N. Basov, J. Feikes, P. Guptasarma, K. Holldack, H.W. Hubers, P. Kuske, Michael C. Martin, W.B. Peatman, U. Schade, G. Wustefeld; "Measuring the Josephson plasma resonance in $\mathrm{Bi} 2 \mathrm{Sr} 2 \mathrm{CaCu} 2 \mathrm{O} 8$ using intense coherent THz synchrotron radiation," Phys. Rev. B 69 (9), 092512 (2004).

J.M. Byrd, Michael C. Martin, W.R. McKinney, D.V. Munson, H. Nishimura, D.S. Robin, F. Sannibale, R.D. Schlueter, W.G. Thur, J.Y. Jung, and W. Wan, "CIRCE: A dedicated storage ring for coherent THz synchrotron radiation," Infrared Physics and Technology 45(5-6), 325-330 (2004). LBNL-53699

ALS-Padmore LB03019 Aberration Correction of Electron Microscopes

H. Rose, "Five-Dimensional Hamilton-Jacobi Approach to Relativitistic Quantum Mechanics," Advances in Imaging and Electron Physics, Vol. 132 (2004).

P. Schmid, et. al., "Alignment Strategies for the Mirror Corrector for the PEEM-3 Microscope," accepted for publication in Reviews of Scientific Instruments.

Weishi Wan et. al., "Design Study on a New Separator for PEEM3," in preparation.

ALS-Schoenlein LB02006 Simulations of Femtosecond X-ray Spectra of Photoexcited Molecules

L. Campbell and S. Mukamel, "Simulation of x-ray absorption near edge spectra of electronically excited ruthenium tris-2,2'-bipyridine," Journal of Chemical Physics, in press (2004).

ALS-Wilcox LB04037 High Average Power Laser Amplifier

R. Wilcox, R. Schoenlein, "A High Average Power, High Repetition Rate, Femtosecond Laser System Using Cryogenically Cooled Ti:sapphire," Conference on Lasers and Electro Optics, abstract submitted.

CH-Ahmed LB04038 Gas Phase Studies of the Building Blocks of Life

C. Nicolas, L. Belau, X. Tang, S. R. Leone \& M. Ahmed, "Single photon ionization of guanine with synchrotron radiation," draft to be submitted to Journal of American Chemical Society.

K. R. Wilson, C.Nicolas, M. Jimenez-Cruz, D. S. Peterka, S. R. Leone \& M. Ahmed, "VUV photoionization mass spectrometry of amino acids with particle impact flash thermal desorption," draft to be submitted to Analytical Chemistry.

K. R. Wilson, C. Nicolas, M. Jimenez-Cruz, S. R. Leone \& M. Ahmed, "Ionization energies of some amino acids," draft to be submitted to Journal of American Chemical Society.

CH-Booth LB02009 Disorder and Multiple Length Scales in Non-Fermi Liquid f-Electron Intermetallics

S.-W. Han, J. S. Gardner, and C. H. Booth, "Structural properties of the geometrically frustrated pyrochlore $\mathrm{Tb}_{2} \mathrm{Ti}_{2} \mathrm{O}_{7}$," Phys. Rev. B 69, 024416, 2004. LBNL-54103.

S.-W. Han, C.H. Booth, E.D. Bauer, J.M. Lawrence, Huang P. H., and Y.Y. Chen, "Disorderinduced Kondo behavior in nanoparticle $\mathrm{CeAl}_{2}$," Proceedings from the International 
Conference on Magnetism; Rome, Italy; July 22 - August 1, 2003. J. Magn. Magn. Mater., 272-276, E101 (2004). LBNL-52531.

M. Daniel, S.-W. Han, C. H. Booth, A. L. Cornelius, E. D. Bauer, and J. L. Sarrao, "Local structure around $\mathrm{Sn}$ in $\mathrm{CeCoIn}_{5-\mathrm{x}} \mathrm{Sn}_{\mathrm{x}}$," submitted to Physica B. LBNL-55461.

C. H. Booth, S.-W. Han, S. Skanthakumar, and J. L. Sarrao, "Lattice disorder and magnetism in $\mathrm{f}$-electron intermetallics (Invited)," Proceedings from At Frontiers of Condensed Matter II workshop, Buenos Aires, Argentina, June 22-26, 2004, Physica B, in press. LBNL-55996.

M. Daniel, E. D. Bauer, S.-W. Han, C. H. Booth, A. L. Cornelius, and J. L. Sarrao, "Perturbing the superconducting planes in $\mathrm{CeCoIn}_{5}$ by Sn substitution," draft to be submitted to Phys. Rev. Lett. LBNL-56725.

S.-W. Han, C.H. Booth, E.D. Bauer, J.M. Lawrence, Huang P. H., and Y.Y. Chen, "Lattice Disorder and Size-Induced Kondo Behavior in $\mathrm{CeAl}_{2}$ and $\mathrm{CePt}_{2+x}$," draft to be submitted to Phys. Rev. Lett.

S.-W. Han, C. H. Booth, J. L. Sarrao, J. D Thompson, "Local order and disorder in nonFermi liquid CeRhRuSi 2 ," draft to be submitted to Phys. Rev. B.

CH-Leone LB02043 Coherent Control and Quantum Information in Polyatomic Molecules

V.G. Stavros, E. Harel and S.R. Leone, "The influence of intense control laser pulses on homodyne-detected rotational wavepacket dynamics in $\mathrm{O}_{2}$ by degenerate four-wave mixing," J. Chem. Phys. in press.

V.G. Stavros and S.R. Leone, "The influence of ion-gratings on rotational wavepacket dynamics observed in $\mathrm{H}_{2}$," draft to be submitted to Chem. Phys. Lett.

CH-Saykally LB03023 Soft X-ray Spectroscopy of Liquid Surfaces

K.R. Wilson, B.S. Rude, J.D. Smith, C.D. Cappa, D.T. Co, R.D. Schaller, M. Larsson, T. Catalano, and R.J. Saykally, "Investigation of volatile liquid surfaces by synchrotron xray spectroscopy of liquid microjets," Review of Scientific Instruments 75, 725-736 (2004).

J.D. Smith, C.D. Cappa, K.R. Wilson, B.M. Messer, R.C. Cohen, and R.J. Saykally, "Energetics of Hydrogen Bond Network Rearrangements in Liquid Water," Science 306, 851-853 (2004).

K.R. Wilson, B.S. Rude, R.D. Schaller, T. Catalano, M. Cavalleri, A. Nilsson, L.G.M. Pettersson, and R..J. Saykally, "X-ray absorption of methanol liquid microjets: surface vs. bulk electronic structure and hydrogen bonding," J. Phys. Chem. B in-press.

B.M. Messer, C.D. Cappa, J.D. Smith, K.R. Wilson, M.K. Gilles, R.C. Cohen and R.J Saykally, "pH dependence of the electronic structure of glycine," J. Phys. Chem. B in press.

C.D. Cappa, J.D. Smith, K.R. Wilson, B.M. Messer, R.C. Cohen, and R.J. Saykally, "Effects of alkali halide salts on the hydrogen bond network of liquid water," submitted to $J$. Phys. Chem. B.

CH-Shuh LB02044 Scientific Investigations and Technique Development of Wet Spectroscopy, High Pressure Photoelectron Spectroscopy, and Scanning Transmission X-ray Microscopy for Molecular Environmental Science

L. Muntean, R. Planques, A. L. D. Kilcoyne, W. Hinsberg, S. R. Leone, and M. K. Gilles, "Soft x-ray imaging of polymer photoresists," to be submitted to J. Vac. Sci. Technol. B, December (2004). 
Steven R. Maria, Lynn M. Russell, Mary K. Gilles, Satish C.B. Myneni, "Organic aerosol growth mechanisms and the climate forcing implications," Science, accepted November (2004).

G. DeStasio, D. Rajesh, P. Casalbore, M. J. Daniels, R. J. Erhardt, B. H. Frazer, L. M. Wiese, K. L. Richter, B. R. Sonderegger, B. Gilbert, S. Schaub, R. J. Cannara, J. F. Crawford, M. K. Gilles, T. Tyliszczak, J. F. Fowler, L. M. Larocca, S. P. Howard, D. Mercanti, M. P. Mehta, and R. Pallini, "Are gadolinium contrast agents suitable for gadolinium neutron capture therapy?" accepted for publication in Neurological Research, September (2004).

I. J. Drake, T. C. N. Liu, M. K. Gilles, T. Tyliszczak, A. L. D. Kilcoyne, D. K. Shuh, Richard A. Mathies, and A. T. Bell, "An in situ cell for characterization of solids by soft X-ray absorption," Rev. Sci. Instrum. 75, 3242-3247 (2004).

CS-Bailey LB02012 Experimental Mathematician's Toolkit

J. M. Borwein and D. H. Bailey, Mathematics by Experiment: Plausible Reasoning in the 21st Century, A K Peters, Natick, MA, 2004.

J. M. Borwein, D. H. Bailey and Roland Girgensohn, Experimentation in Mathematics: Computational Paths to Discovery, A K Peters, Natick, MA, 2004.

D. H. Bailey, "Java Meets Numerical Analysis: Review of 'Java Number Cruncher' by Ronald Mak," Scientific Programming, accepted for publication. Url: http://crd.lbl.gov/ dhbailey/dhbpapers/dhb-java.pdf.

D. H. Bailey, "Highly Parallel, High-Precision Numerical Integration," manuscript, Aug. 2004. Url: http://crd.lbl.gov/ dhbailey/dhbpapers/quadparallel.pdf.

D. H. Bailey, J. M. Borwein, V. Kapoor and E. Weisstein, "Ten Problems in Experimental Mathematics," manuscript, Sep. 2004. Url: http://crd.lbl.gov/ dhbailey/dhbpapers/tenproblems.pdf.

CS-Crivelli LB02013 Infrastructure for Improving Protein Structure Prediction in Computational Biology

S. Crivelli, O. Kreylos, B. Hamann, N. Max, W. Bethel; "ProteinShop: A Tool for Interactive Protein Manipulation and Steering," J. Computer-Aided Molecular Design, Vol. 18 (2004) pp. 271-285.

S. Crivelli, Ting-Cheng Lu, O. Kreylos, N. Max, W. Bethel; "ProteinShop: A Tool for Protein Manipulation," Proc. $12^{\text {th }}$ Intl. Conf. On Intelligent Systems for Molecular Biology (ISMB 2004), Special Interest Group (3DSIG), 2004. Url: http://3dsig.weizmann.ac.il/3dsig/2004/abstracts/allabs.html

J. Ding, E. Eskow, Ting-Cheng Lu, W. Liu, L. Jiang, R. Byrd, R. Schnabel, and S. Crivelli; "Protein Structure Prediction Using Physics-Based Global Optimization with Knowledge-Guided Fragment Packing," accepted for publication in J. Moult, K. Fidelis, A. Zemla, and T. Hubbard, eds., Proceedings of CASP6 - Sixth Meeting on the Critical Assessment of Techniques for Protein Structure Prediction, Gaeta, Italy, December 2004. Url: http://www.forcasp.org/modules.php?name=Papers\&file=article\&sid $=2187$

C. Crawford, W. Bethel, B. Hamann, O. Kreylos, N. Max, R. Oliva, S. Crivelli; "Visualization of Energy Optimization," (poster). SIAM Conference on the Life Sciences, 2004. 
CS-Ding LB02014 New Machine Learning and Data Mining Methods for Genomics and Climate Data Analysis

Chris Ding, Xiaofeng He, Richard Meraz, Steve Holbrook, "A Unified Representation for Multi-Protein Complex Data for Modeling Protein Interaction Networks," Proteins: Structure, Function, and Bioinformatics, 57:99-108, June 2004.

Richard Meraz, Xiaofeng He, Chris Ding, Steve Holbrook, "Positive Sample Only Learning (PSOL) for Predicting RNA Genes in E. coli.," Proc. IEEE Computer Society Bioinformatics Conference, pp.535-538, August 2004. Stanford, CA.

Chris Ding and Xiaofeng He, "K-means Clustering via Principal Component Analysis," Proc. of Int'l Conf. Machine Learning (ICML 2004), pp 225-232. July 2004.

Chris Ding and Xiaofeng He, "Linearized Cluster Assignment via Spectral Ordering," Proc. of Int'l Conf. Machine Learning (ICML 2004), pp.233-240. July 2004.

Chris Ding, Hongyuan Zha, Xiaofeng He, Parry Husbands and Horst D. Simon, "Link Analysis: Hubs and Authorities on the World Wide Web," SIAM Review v.46, pp.256268, June 2004.

Hanchuan Peng and Chris Ding, "Structure Search and Stability Enhancement of Bayesian Networks," Proc. IEEE Int'l Conf. Data Mining, pp.621-624, Melbourne, Florida, Nov 2003.

Chris Ding and Jieping Ye, "Two-dimensional Singular Value Decomposition for 2D maps and images," accepted for publication in SIAM Data Mining Conference 2005.

CS-Hamann LB03012 Interactive Visualization Methods for Exploration and Comparison of Multi-Billion Base Pair Sequence Data

N. Shah, A. Poliakov, D. Ryaboy, M. Teplitsky, B. Hamann, E. Rubin, and I. Dubchak, "VISTA tools for interactive visualization and analysis of multiple alignments of data sequences and whole genomes, poster presentation," in: Hudson, T.J., Paabo, S., Rogers, J. and Rubin, E.M., eds., Proceedings of The Biology of Genomes, Cold Spring Harbor Laboratory Press, Woodbury, New York, 2004.

N. Shah, S. Dillard, G. Weber, B. Hamann, "Volume visualization of multiple alignment of large genomic DNA," submitted to IEEE Trans. on Visualization and Computer Graphics (TVCG).

CS-Meza LB02045 Parallel Methods for Robust Optimization and Uncertainty Quantification

J.C. Meza, R.A. Oliva, P.D. Hough, P.J. Williams, "OPT++: An object-oriented nonlinear optimization framework," submitted to ACM Transactions on Mathematical Software.

J.C. Meza, R.A. Oliva, Best Poster Award, SIAM Conference on Parallel Processing for Scientific Computing, San Francisco, CA, Feb. 25-27, 2004.

J.C. Meza, R.A. Oliva, "A C++ Class for Managing Schittkowski 's Collection of Nonlinear Optimization Test Problems," in Proceedings of Tapia Celebration of Diversity in Computing Conference, October 2003.

CS-Pinar LB02018 Combinatorial Algorithms in Scientific Computing

A. Pinar, T. Tao, H. Ferhatosmanoglu, "Efficient Indexing for Scientific Databases," accepted for publication in the Proc. IEEE International Conference on Data Engineering, Japan, 2005.

A. Pinar, E. Chow, A. Pothen, "Combinatorial Techniques for Constructing Sparse Nullspace Bases," submitted to Electronic Transactions on Numerical Algorithms, special issue on Saddle-point Problems. 
A. Pinar, B. Hendrickson, "Combinatorial Parallel and Scientific Computing," chapter to appear in Frontiers on Scientific Computing, SIAM.

A. Pinar, V. Vassilevska, "Finding Dense Blocks of a Sparse Matrix," submitted to Electronic Transactions on Numerical Algorithms, special issue on Combinatorial Scientific Computing.

A. Pinar, and A. Pothen, "The Nice Basis Problem," in preparation.

A. Pinar and B. Hendrickson, "Improving Load Balance by Exploiting Flexible Assignable Work," submitted to IEEE Transactions of Parallel and Distributed Computing.

A. Pinar and B. Hendrickson, "Interprocessor Communication with Limited Memory," IEEE Transactions on Parallel and Distributed Systems, 15,7: 606--616, 2004.

A. Pinar and C. Aykanat, "Fast Optimal Load Balancing Algorithms for 1D Partitioning," Journal of Parallel and Distributed Computing, 64: 974--996, 2004.

A. Pinar L. Fleischer, B. Hendrickson, "A Divide-and-Conquer Algorithm for Identifying Strongly Connected Components," submitted to Networks.

A. Pinar and B. Hendrickson, "Exploiting Flexibly Assignable Work to Improve Load Balance," Proc. ACM 14th Symp. Parallel Algorithms and Architectures (SPAA) 2002, pages: $155-163$.

CS-Rescigno LB04029 Advanced Computational Methods for Photon-Molecule Collision Processes

C. W. McCurdy and F. Martin, "Implementation of Exterior Complex Scaling in B-Splines to Solve Atomic and Molecular Collision Problems," J. Phys. B 37, 917 (2004).

D. A. Horner, J. Colgan, F. Martin, C. W. McCurdy, M. S. Pindzola and T. N. Rescigno, "Symmetrized Complex Amplitudes for He Double Photoionization from the TimeDependent Close Coupling and Exterior Complex Scaling Methods, " accepted for publication in Phys. Rev. A.

W. Vanroose, F. Martin, T. N. Rescigno and C. W. McCurdy, "Nonperturbative Theory of Double Photoionization of the Hydrogen Molecule," submitted for publication in Phys. Rev. A.

CS-Yelick LB03030 Evaluation of Computer Architecture Alternatives

M. Narayanan, "Compiling Communication Access Patterns for a Vector Processor," Master's Report, Computer Science Division, University of California, Berkeley. 2004.

G. Griem, L. Oliker, J. Shalf and K. Yelick, "Identifying Performance Bottlenecks on Modern Microarchitectures Using an Adaptable Probe," Third International Workshop on Performance Modeling, Evaluation, and Optimization of Parallel and Distributed Systems (PMEO-PDS) 2004. Url: http://crd.lbl.gov/ oliker/papers/pmeo_2004.pdf

L.Oliker, P.Husbands, G.Griem, J.C.Lawrence, "Evaluation of Architectural Paradigms for Addressing the Processor-Memory Gap," submitted to HPCA-10.

M. Narayan and K. Yelick, "Generating Permutation Instructions from a High-Level Description," 6th Workshop on Media and Streaming Processors, December 2004

S.Kamil, J. Shalf, P. Husbands, L. Oliker, K. Datta, P Hargrove, K. Yelick, "Optimizing Stencil Computatioins on Modern Architectures," submitted to IEEE International Symposium on Performance Analysis of Systems and Software (ISPASS) 2005. Url: http://www.cs.berkeley.edu/ skamil/ISPASS05.pdf

S. Kamil, J. Shalf, K. Yelick et al., "Simulating the HPCC Benchmarks Using an Adaptable Probe," in preparation. 
ESD-Alvarez-Cohen LB03008 Application of Real-time PCR with Reverse Transcription for Quantification of Specific Microbial Activity in Complex Communities

D. R. Johnson, P. K. H Lee., V. F. Holmes and L. Alvarez-Cohen, "An internal reference technique for quantifying specific mRNAs by real-time PCR with application to the tceA reductive dechlorination gene," submitted to Applied and Environmental Microbiology.

B. G. Rahm, V. F. Holmes, Chauhan, S., T. W. Macbeth, K. S. Sorenson and L. AlvarezCohen, "Molecular Characterization of Microbial Populations at Two Sites with Differing Reductive Dechlorination Abilities," submitted to Environmental Science and Technology.

D. R. Johnson, P. K. H. Lee, V. F. Holmes and L. Alvarez-Cohen, "Environmental factors affecting the expression of the tceA reductive dechlorination gene in an anaerobic microbial enrichment culture," in preparation.

V. F. Holmes, J. Z. He, D. R. Johnson and L. Alvarez-Cohen, "Assessing Reductive Dechlorination Potential by Quantifying Dehalococcoides Reductase Genes," Superfund Basic Research Program Annual Meeting, Seattle, WA. 2004.

V. F. Holmes and L. Alvarez-Cohen, "Growth and Dechlorination Activity of Dehalococcoides ethenogenes 195 within Assembled Co- and Tri-Cultures," $104^{\text {th }}$ general meeting of American Society for Microbiology, New Orleans, LA. 2004.

D. R. Johnson, V. F. Holmes and L. Alvarez-Cohen, "Development of an Internal Reference Technique for the Quantification of mRNA by qPCR with Application to the tceA Reductive Dechlorination Gene," 104th general meeting of American Society for Microbiology, New Orleans, LA. 2004.

ESD-Banfield LB02020 Microbial Controls on Metals in the Environment

G.K. Druschel, B.J. Baker, T.H. Gihring, J.F. Banfield, "Acid mine drainage biogeochemistry at Iron Mountain, California," Geochemical Transactions 5, 13-32. (2004)

B.J. Baker, M.A. Lutz, M.A., S.C. Dawson, P.L Bond, and J.F. Banfield, "Metabolically active eukaryotes in extremely acidic mine drainage," Appl. Environ. Microbiol. 70, 6264-6271. (2004).

G.W. Tyson, J. Chapman, P. Hugenholtz, E. Allen, R.J. Ram, R.J., P. Richardson, V. Solovyev, Rubin, D. Rokhsar, and J.F. Banfield, "Insights into microbial community structure and metabolism by reconstruction of genomes from a natural environment," Nature, 428, 37 - 43 (2004).

Lo, G.W. Tyson, P. Hugenholtz, and J.F. Banfield, "Population structure of Leptospirillum in an acid mine drainage microbial community," draft to be submitted to Applied and Environmental Microbiology.

G.W. Tyson, I, Lo, B.J. Baker, E.E. Allen, P.Hugenholtz, and J.F. Banfield, "GenomeDirected Isolation of the Key Nitrogen Fixer, Leptospirillum ferrodiazotrophum sp. nov., from an Acidophilic Microbial Community," submitted to Applied Env. Microbiol.

J. Flanagan, B. Baker, J.F. Banfield, "Characterization of Arsenate Reductase from an acidophilic ultra-small archaeon," in preparation.

ESD-Bodvarsson LB03004 Comparative Studies Between Earth and Planetary Science

G. Brimhall, "Resource Perspective on Potentially Cost-effective Unconventional Solar Cells," draft to be submitted to Progress in Photovoltaics: Research and Applications. 
ESD-Borglin LB04005 Development of Biosensors for Endocrine Disrupting Compounds in Agricultural Watersheds

C. Campbell, S. Borglin, W. Stringfellow, F.B.Green, A. Grayson, "Review of bioassays for monitoring fate and transport of estrogenic endocrine disrupting compounds in water," submitted to Rev. in Env. Sci. and Tech. Url: http://wwwlibrary.lbl.gov/docs/LBNL/561/37/PDF/LBNL-56137.pdf

ESD-Miller LB04025 California Water and Energy System: An Approach for Addressing Future Crises

L.D. Brekke, N.L. Miller, K.E. Bashford, N.W.T. Quinn, and J.A. Dracup, "Climate change impacts uncertainty for water resources in the San Joaquin River Basin, California," $J$. Amer. Water Resources Assoc., No. 02103, Feb. 2004, LBNL 51393.

L. Dale, C. Dunham Whitehead, A. Fargeix, "Electricity Price and Southern California's Water Supply Options," Resources, Conservation, and Recycling. LBNL-53056.

J. Jin and N.L. Miller, "An analysis of climate variability in mountainous regions," submitted to J. Hydrometeorology. LNBL-53845.

J. Jin, N.L. Miller, S. Sorooshian, and X. Gao, "Relationship between atmospheric circulation and snowpack in the western United States," submitted to $J$. Hydrometeorology. LBNL-55404.

K. Hayhoe, D.C. Cayan, C.B. Field, P.C. Frumhoff, E.P. Mauer, N.L. Miller, S.C. Moser, S.H. Schneider, K. Nicholas Cahill, E.E. Cleland, L.D. Dale, F. Davis, R. Drapek, R.M. Hanemann, L.S. Kalkstein, J. Lenihan, C.K. Lunch, R.P. Neilson, S.C. Sheridan, J.H. Verville, "Emissions pathways, climate change, and impacts on California," Proceedings of the National Acad. Sciences, 101, 12422-12427, Aug. 2004. LBNL-56119.

P.C. Kyriakidis, N.L. Miller, and J. Kim, "A Spatial Time Series Framework for Modeling Daily Precipitation at Regional Scales," Journal of Hydrology. 297(1-4):236-255, September 2004. LBNL-49156.

R.M. Maxwell and N.L. Miller, "On the development of a coupled land surface and groundwater model for use in watershed management," in press J. Hydrometeorology. LBNL-55029.

N.L. Miller, "California Climate Change, Hydrologic Response, and Flood Forecasting," Chap. 10. in Urban Flood Management, Ed. A. Szollosi-Nagy and C. Zevenbergen, Cheriton House Pub., November 2004. LBNL-54041.

N.L. Miller, A.W. King, M.A. Miller, E.P. Springer, M.L. Wesely, K.E. Bashford, M.E. Conrad, K. Costigan, P.N. Foster, H.K. Gibbs, J. Jin, J. Klazura, B.M. Lesht, M.V. Machavaram, F. Pan, J. Song, D. Troyan, R.A. Washington-Allen; "The DOE Water Cycle Pilot Study," in press Bulletin of the Amer. Meteorological Soc. LBNL-53826.

N.W.T. Quinn, L.D. Brekke, N.L. Miller, T. Heinzer, H. Hildago, J.A. Dracup, "Model integration for assessing future hydroclimate impacts on water resources, agricultural production and environmental quality in the San Joaquin Basin, California," Environ. Modelling and Software, 19, 305-316, 2004.

ESD-Sposito LB04034 Advancing the Next Generation of Rock-Fluid Imaging and Stimulation Technologies

W.-C. Lo, G. Sposito, E. Majer, "Wave propagation through porous media containing two immiscible fluids," American Geophysical Union Annual Fall Meeting (December 2003). 
W.-C. Lo, G. Sposito, E. Majer, "Low-frequency dilatational wave propagation through unsaturated porous media containing two immiscible fluids," American Geophysical Union Annual Fall Meeting (December 2004).

W.-C. Lo, G. Sposito, E. Majer, "Wave propagation through an elastic porous medium containing two immiscible fluids," Water Resources Research in press.

W.-C. Lo, G. Sposito, E. Majer, "Low-frequency dilatational wave propagation through unsaturated porous media containing two immiscible fluids," submitted to Transport in Porous Media.

W.-C. Lo, G. Sposito, E. Majer, "Dynamic response of a saturated porous medium to pore pressure pulsing at seismic frequencies," draft to be submitted to Water Resources Research.

ESD-Waychunas LB02026 Reactivity of Nanoparticles in Natural Environments

B Gilbert, F Huang, H Zhang, G Waychunas, and J Banfield, "Nanoparticles: strained and stiff," Science, 305, 651-654 (2004).

B Gilbert, H Zhang, F Huang, Y Ren, D Haskel, J Lang, G Srajer, A Jürgensen, G Waychunas, J Banfield, "Analysis and simulation of nanoparticle structures observed in a surface-driven structural transformation," Journal of Chemical Physics 120, 1178511795 (2004).

C Kim, G Waychunas, J Banfield, "Effects of aggregation-based growth on heavy metal sorption and precipitation onto nanoscale iron oxyhydroxides," invited paper to be submitted to Journal Colloid Interface Science.

G Waychunas, C Kim, J Banfield, "Nanoparticulate oxide minerals in soils and sediments: unique properties and contaminant scavenging mechanisms," invited paper to be submitted to Journal of Nanoparticle Research.

$\mathrm{H}$ Zhang, J Banfield, "Aggregation, coarsening, and phase transformation in $\mathrm{ZnS}$ nanoparticles studied by molecular dynamics simulations," Nanoletters, 4, 713-718 (2004).

H Zhang, F Huang, B Gilbert, J Banfield, "Reversible, surface-controlled structure transformation in nanoparticles induced by aggregation-disaggregation," Physical Review Letters, 92, (15) electronic identifier 155501 (2004).

H Zhang, F Huang, B Gilbert, J. Banfield, "Molecular dynamics simulations, thermodynamic analysis and experimental study of phase stability of zinc sulfide nanoparticles," Journal of Physical Chemistry B, 107 (47), 13051 -13060 (2003).

EETD-Apte LB02028 Miniaturized Systems for Particle Exposure Assessment

MG Apte, DR Black, LA Gundel, and AD Hansen, 2002. Miniaturized System for Particle Exposure Assessment. Provisional Patent Application 1850P, Submitted to US. Patent Office, April, 2003.

MG Apte, LA Gundel, DR Black, and AD Hansen, 2004. Apparatus for Particulate Matter Analysis. Patent Application, Attorney Docket No.: IB-1850. Assignee: The Regents of the University of California. Filed with US. Patent Office, May 14, 2004.

EETD-Kirchstetter LB03015 Determining the Light-Absorbing Properties of Aerosol Particles

T. Kirchstetter, T. Novakov, P. Hobbs, "Evidence that Spectral Light Absorption by Aerosols Emitted from Biomass Burning and Motor Vehicles is Different due to Organic Carbon," Journal of Geophysical Research 109, November 2004. Url: http://www.agu.org/pubs/crossref/2004/2004JD004999.shtml 
EETD-Mao LB04021 Nanostructured Cathodes for Efficient Organic LEDs

S. S. Mao, G. Liu, R. E. Russo, and S. Johnson, "Threshold Current Reduction of Organic LEDs Using Nanostructured Electrodes," draft to be submitted to Applied Physics Letters.

EETD-Menon LB04023 Evaluating Aerosol Effects on Regional and Global Energy and Water Budgets

N. Chmura and S. Menon, "Analyzing Signatures of Aerosol-Cloud Interactions detected by MODIS," submitted to Geophys. Res. Lettr.

S. Menon and A.D. Del Genio, "Evaluating the impacts of carbonaceous aerosols on clouds and climate," in review for Human-induced climate change: An interdisciplinary Assessment, edited by Schlesinger et al.

S. Menon, "Current uncertainties in aerosol climate effects," Annual Reviews 29, 1-30.

Y. Qian, Q. Song, S. Menon, S. Yu, S. Liu, G. Shi, R. Leung, and Y. Luo, “Impacts of atmospheric sulfate and black carbon aerosols on the regional climate of East Asia," in press for Global Environmental Change Research in East Asia.

EETD-Sathaye LB04043 Long Term Global Energy Demand and Carbon Emissions Scenarios

J. Sathaye, "Bottom-up Modeling of Energy and Greenhouse Gas Emissions: Approaches, Key Results and Challenges," submitted for book chapter in Integrated Assessment of Human Induced Climate Change, Cambridge University Press. 2005.

J. Sathaye, "Bottom-up Modeling of Energy and Greenhouse Gas Emissions: Approaches, Key Results and Challenges," paper presented to Energy Modeling Forum, Snowmass, Colorado, August 4, 2004.

J. Sathaye, "Energy and Greenhouse Gas Emissions Modeling: Scenarios and Mitigation Costs," paper presented at the APERC Energy Outlook Workshop, Tokyo, Japan. September 15, 2004.

EETD-Van Buskirk LB03027 Evaluation of Dynamic Air Quality Impacts of Distributed Generation

T. Ghidey, "MM5 simulations of mesoscale transport from the SFBA to Sacramento and the SJV during CCOS, December 2004," accepted in partial fulfillment of the requirements of the degree Masters of Science.

GN-Myers LB04026 Making the Most of Sequencing: Improved Assembly and Comparative Annotation

K. Rasmussen, J. Stoye, G. Myers, "Efficient q-gram filters for finding all $\varepsilon$-matches over a given length," submitted to RECOMB conference, would appear April 2005.

R. Edgar, C. Smith, S. Celniker, G. Myers, "PILER: computational identification of novel interspersed repeats," to be submitted to Genome Research.

G. Myers, "A string graph approach to fragment assembly," in preparation.

GN-Rubin LB03034 Identification and Characterization of Conserved Noncoding Sequences Using Comparative Genomics and Transgenic Technology

L.A. Pennacchio, N. Baroukh, E.M. Rubin, "Human-Mouse Comparative Genomics: Successes and Failures to Reveal Functional Regions of the Human Genome," Cold Spring Harb Symp Quant Biol. 2003. 
F. Poulin, M. Nobrega, I. Plajzer-Frick, A. Holt, V. Afzal, E.M. Rubin, and L.A. Pennacchio, "In Vivo Characterization of a Vertebrate Ultra-conserved Enhancer," submitted to Genome Research.

N. Baroukh, E. Bauge, J. Akiyama, J. Chang, V. Afzal, J.C. Fruchart, E.M. Rubin, J. Fruchart-Najib, L.A. Pennacchio, "Analysis of Apolipoprotein A5, c3, and Plasma Triglyceride Concentrations in Genetically Engineered Mice," Arterioscler Thromb Vasc Biol. (2004).

M.A. Nobrega, Y. Zhu, I. Plajzer-Frick, V. Afzal, E.M. Rubin, "Megabase Deletions of Gene Deserts Result in Viable Mice," Nature 431: 988-993 (2004).

LSD-Auer LB04010 Molecular Microscopy and Tomography

Manfred Auer, Bram Koster, Ulrike Zlese, Chandajit Bajaj, Niels Volkmann, Da Neng Wang \& A. James Hudspeth, "Architecture Of Hair Cell Ankle, Kinociliar And Tip Links, As Revealed By Electron Tomography," draft to be submitted to Journal of Neuroscience.

M. Auer, A. J. Hudspeth, "Visualizing Hair Bundles In Three Dimensions At Molecular Resolution: Extracellular Links, The Adaptation Machinery, And Lipid Rafts," submitted to Association for Research in Otolaryngology - Midwinter Meeting 2/2005.

Hildur Palsdottir, Manfred Auer, "3D Electron Microscopy Tomographic Visualization Of Lipid Rafts," accepted for oral presentation at Biophysical Society Meeting 2/2005.

LSD-Cooper LB04006 Analysis of Complex Phosphorylation Patterns in a Key DNA Repair Protein by Coupling Surface Plasmon Resonance ...

One article to be prepared.

LSD-Dernburg LB01024 Dynamic Reorganization of Chromosome Architecture During Meiosis

A. MacQueen, A. Villeneuve, N. Bhalla, A. Dernburg, "Cis-acting sites required for synaptic initiation in C. elegans," draft to be submitted to Cell.

C. Phillips, C. Wong, N. Bhalla, P. Meneely, and A. Dernburg, "A chromosome-specific homolog pairing factor in C. elegans meiosis," draft to be submitted to Cell.

Needhi Bhalla and Abby F. Dernburg, "Chromosome synapsis is monitored by a doublestrand-break-independent checkpoint during meiosis in C. elegans," draft to be submitted to Developmental Cell.

Peter M. Carlton and Abby F. Dernburg, "A link between meiotic nuclear architecture and crossover control," draft to be submitted to Current Biology.

LSD-Karpen LB03032 Identification and Analysis of Determinants of Centromere Identify in Drosophila

B.A. Sullivan and G.H. Karpen, "Centromeric chromatin displays a histone modification pattern that is distinct from euchromatin and heterochromatin," Nature Structural and Molecular Biology 11, 1076-1083.

P. Heun, S.E. Erhardt, M.D. Blower, A. Skora, and G.H. Karpen, "Drosophila CENP-A / CID is sufficient for kinetochore formation at ectopic sites," submitted.

M.D. Blower, T. Daigle, T. Kaufman, and G.H. Karpen, "CENP-A mutations in Drosophila cause an early mitotic delay that involves a component of the Spindle Assembly Checkpoint," submitted. 
LSD-Martin LB04022 Imaging Three-dimensional Signaling Networks in Normal and Malignant Tissue

R. Berdeaux, B. Diaz, L.C. Kim, G.S. Martin, "Active Rho is localized to podosomes induced by oncogenic Src and is required for their assembly and function," J. Cell Biol. 166: 317-323 (2004). Url: http://www.jcb.org/cgi/content/full/166/3/317.

\section{LSD-Mian LB02031 Systems Biology: Biological Input-Output Devices}

Calder, R.B., Garza, J.D., Beems, R., van Steeg, H., Hoeijmakers, J., Lohman, P.H.M., Mian, I.S. \& Vijg, J.; "A Mouse Phenotype Analysis System (MPHASYS),” in preparation (2004).

Semeiks, J. and Mian, I.S., "Large complex networks of genes, documents and interacting proteins: application to telomere homeostasis, in preparation (2004).

Semeiks, J., Rizki, A., Bissell, M.J. \& Mian, I.S., "Ensemble attribute profile clustering: discovering and characterizing groups of genes with similar patterns of structural and functional features," in preparation (2004).

Lelievre, S., Weaver, V.M., Abad, P.C., Mian, I.S. \& Bissell, M.J.; "NuMA acts a functional link between integrin adhesion and nuclear structure to regulate survival in breast epithelium," in preparation (2004).

Nakayama, Y., Ogawa, T., Mian, I.S. and Kohwi-Shigematsu, T.; "A novel nuclear localization signal is located in the N-terminus of SATB1," submitted to J. Biol. Chem.

Rizki, A., Weaver, V.M., Chin, K. Moonlee, S.-Y., Rozenberg, G.I., Myers, C.A., Bascom, J.L., Mott, J.D., Semeiks, J.R., Grate, L.R., Mian, I.S., Petersen, O.W., Chen, D.J., Chen, F., Gray, J.W. \& Bissell, M.J.; "Identification of functionally significant changes in transition from premalignant to malignant phenotype," submitted to Cancer Cell.

Wu, W., Xing, E.P., Myers, C.A., Mian, I.S. and Bissell, M.J.; "Evaluation of normalization methods for cDNA microarray data by classification," submitted to Genome Biology.

Eydmann, T., Sommariva, E., Mian, I.S., Klar, A. \& Dalgaard, J.; "A chromo-like domain mediates DNA replication termination function of the novel protein, rtflp," submitted to PNAS.

Blei, D.M., Franks, K., Jordan, M.I. \& Mian, I.S.; "Statistical modelling of biomedical corpora: mining the Caenorhabditis Genetic Center Bibliography," to appear in Mechanisms of Ageing \& Development.

Reed, J.A., Lin, Q., Chen, D., Mian, I.S. \& Medrano, E.E.; "SKI pathways inducing progression in human melanoma," to appear in Cancer \& Metastasis Reviews.

Semeiks, J.R., Grate, L.R. \& Mian, I.S.; "Text-based analysis of genes, proteins, aging, and cancer," to appear in Mechanisms of Ageing \& Development.

Bhattacharyya, C., Grate, L.R., Jordan, M.I., El Ghaoui, L. \& Mian, I.S.; "Robust sparse hyperplane classifiers: application to uncertain molecular profiling data," to appear in $J$. Comp. Biol.

Chen, D., Lin, Q., Mian, I.S., Reed, J. \& Medrano, E.E.; "The multiple roles of the oncogenic protein SKI in human malignant melanoma, " in From Melanocytes to Malignant Melanoma, Eds. Hearing, V. and Leong, S., Humana Press (2004).

Semeiks, J.R., Grate, L.R. \& Mian, I.S.; "Networks of genetic loci and the scientific literature," to appear in International Conference on Complex Systems 2004.

Abad, P.C., Mian, I.S., Plachot, C., Nelpurackal, A., Bator-Kelly, C. \& Lelievre, S.A.; "The C-terminus of the nuclear protein NuMA: phylogenetic distribution and structure," Protein Science 13:2573-2577 (2004). 
Loguinov, A.V, Mian, I.S. \& Vulpe, C.D.; "Exploratory differential gene expression analysis in microarray experiments with no or limited replication," Genome Biology 5:R18 (2004).

Bissell, M.J., Rizki, A. \& Mian, I.S.; "Tissue architecture: the ultimate regulator of breast function," Current Opinions in Cell Biology 15:753-762 (2003).

LSD-Ortiz De Solórzano LB03024 Characterization of Adult Stem Cell Involvement in Mammary Gland Development

R. Fernández-González, T. Deschamps, A. Idica, R. Malladi, C. Ortiz de Solórzano, "Automatic segmentation of histological structures in mammary gland tissue sections," Journal of Biomedical Optics 9(3), 444-453, 2004.

R. Fernández-González, M.H. Barcellos-Hoff, C. Ortiz de Solórzano, "Quantitative image análisis in Mammary gland biology," accepted for publication in Journal of Mammary Gland Biology and Neoplasia.

R. Fernández-González, M.H. Barcellos-Hoff, C. Ortiz de Solórzano, "A tool for the quantitative spatial análisis of complex cellular systems," submitted to IEEE Transactions on Medical Imaging.

O. Laribi, Idica A., M.H. Barcellos-Hoff, C. Ortiz de Solórzano, "Do bone marrow cells transdifferentiate into mouse mammary gland?" draft to be submitted to Breast Cancer Research.

MSD-Bertozzi LB03002 Molecular Control of Interfaces Between Biological and Synthetic Materials

C.M. Klapperich, C.R. Bertozzi, "Global Gene Expression Patterns of Cells Attached to a Tissue Engineering Scaffold," Biomaterials 25, 5631-5641 (2004).

J. Song, J.S. Cisar, C.R. Bertozzi, "Functional Self-Assembling Bolaamphiphilic Polydiacetylenes as Colorimetric Sensor Scaffolds," J. Am. Chem. Soc. 126, 8459-8465 (2004).

J. Song, J. Chen, C.M. Klapperich, V. Eng, C.R. Bertozzi, "Functionalized Glass Slides for in vitro Evaluation of Interactions Between Osteosarcoma TE85 Cells and Mineral-Binding Ligands," J. Mat. Chem 14, 2643-2648 (2004).

R. Parthasarathy, J.T. Groves, "Protein patterns at lipid bilayer junctions," Proc. Natl. Acad. Sci. USA, 101, 2798-803 (2004).

MSD-Dubon LB04008 Directed Assembly of Germanium Island Arrays on Gold-Patterned $\underline{\operatorname{Si}(100)}$

J.T. Robinson, J.A. Liddle, A. Minor, V. Radmilovic, and O.D. Dubon, "Directed assembly of Ge islands grown on Au-patterned Si(100)," accepted for publication in Proceedings of the 27th International Conference on the Physics of Semiconductors, Flagstaff, July 2630, 2004.

MSD-Francis/Trauner LB04036 Development of Light-Switchable Potassium Channels Banghart M., Borges, K.; Isacoff. E., Trauner, D.; Kramer, R.H.; "Light-Activated Potassium Channels for Remote Control of Neuronal Firing," Nature Neurosci. 7, 1381 (2004). 
MSD-Long LB04020 Extending Electron Delocalization in Mixed-Valent Molecular Assemblies

L. Berben, N. Crawford, J. Long, " $\left[(\text { Cyclen })_{3} \mathrm{Ru}_{3}(4,4 \text { '-bpy })_{3}\right]^{7+}$ : A Triangular Complex with a Remarkably Stable $\mathrm{Ru}^{\mathrm{II}}-\mathrm{Ru}^{\text {II }}$-Ru ${ }^{\text {III }}$ Mixed-Valent State," draft to be submitted to $J$. $A m$. Chem. Soc.

MSD-Moore LB03017 Modeling Quantum Coherence and Transport in Nanoscale Spin, Charge, and Flux Devices

J. E. Moore and D. H. Lee, "Geometric effects on T-breaking in $\mathrm{p}+\mathrm{ip}$ and $\mathrm{d}+\mathrm{id}$ superconducting arrays," Phys. Rev. B 69, 104511 (2004).

V. Aji, J.E. Moore, and C.M. Varma, "Higher harmonics of electronic-vibrational coupling in sub-resonant and resonant transport in a single-molecule device," Int. J. Nano. 3, 255 (2004).

C. Xu and J. E. Moore, "Strong-weak coupling self-duality in the two-dimensional quantum phase transition of $\mathrm{p}+\mathrm{ip}$ superconducting arrays," Phys. Rev. Lett. 93, 047003 (2004).

A. Vishwanath, J. E. Moore, and T. Senthil, "Screening and dissipation at the superconductor-insulator transition induced by a metallic ground plane," Phys. Rev. B 69, 054507 (2004).

MSD-Suzuki LB04035 Magnetism at the Nanometer Scale in Spin Polarized Materials

D. Dale, A. Fleet, J.D. Brock, Y. Suzuki, "In-situ X-ray diffraction studies of manganite thin film growth," draft to be submitted to Phys Rev B.

Yayoi Takamura, Jostein K. Grepstad, Rajesh V. Chopdekar, Ann F. Marshal, Hong Zheng, John F. Mitchell, and Yuri Suzuki, "Structural, magnetic and electronic properties of (110)-oriented epitaxial thin films of the bilayer manganite $\mathrm{La}_{1.2} \mathrm{Sr}_{1.8} \mathrm{Mn}_{2} \mathrm{O}_{7}$," draft to be submitted to App Physics Lett.

NSD-Bedaque LB02039 Effective Field Theory and Few-Nucleon Systems

Paulo F. Bedaque, Jiunn-Wei Chen, "Twisted Valence Quarks and Hadron Interactions on the Lattice," LBNL-56738, e-Print Archive: hep-lat/0412023.

Paulo F. Bedaque, Harald W. Griesshammer, Gautam Rupak, "A Nucleon in a Tiny Box," ePrint Archive: hep-lat/0407009.

Paulo F. Bedaque, "Aharonov-Bohm Effect and Nucleon Nucleon Phase Shifts on the Lattice," Phys. Lett. B 593:82-88 (2004).

S.R. Beane, P.F. Bedaque, A. Parreno, M.J. Savage, "Two Nucleons on a Lattice," Phys. Lett. B 585:106-114 (2004).

NSD-Lesko LB04017 Research and Development for Double Beta Decay Experiments

R. Henning for the Majorana Collaboration, "The Simulation of the Majorana Neutrinoless Double-beta Decay Experiment," poster presentation at Neutrino 2004, Paris, France, June, 2004 and Proc. Neutrino 2004 (Paris) in press. Url: http://neutrino2004.in2p3.fr/

R.Henning for the Majorana Collaboration, "Simulation for the Majorana Zero Neutrino Double-Beta Decay Experiment," DNP Fall Meeting Chicago BAPS CG.014, Oct 27-30, 2004. Url: http://www.aps.org/meet/DNP04/baps/index.html

T. Gutierrez, CUORE/Cuoricino Collaboration, "An update on the neutrinoless double beta decay search at Cuoricino," DNP Fall Meeting Chicago BAPS CG.007, Oct 27-30, 2004. Url: http://www.aps.org/meet/DNP04/baps/index.html 
C. Arnaboldi et al., "CUORE: a cryogenic underground observatory for rare events," Nucl. Instrum. \& Meth. A 518: 775 (2004).

C. Arnaboldi et al., "First results on neutrinoless double beta decay of Te-130 with the calorimetric CUORICINO experiment," Phys. Lett. B 584: 260 (2004).

E. B. Norman et al., "Cosmic-ray production of Co-60 in double beta decay source materials," Proc. Neutrino 2004 (Paris) in press. Url: http://neutrino2004.in2p3.fr/

NSD-Wieman LB02048 Research on a Next Generation Vertex Detector

S. Kleinfelder, F. Bieser, Y. Chen, R. Gareus, H.S. Matis, M. Oldenburg, F. Retiere, H.G Ritter, H.H. Wieman, E. Yamamoto, "Novel integrated CMOS sensor circuits," IEEE Transactions on Nuclear Science 51, Issue: 5, pp 2328-2336 (2004).

H.S. Matis, F, Bieser, Y. Chen, R. Gareus, S. Kleinfelder, M. Oldenburg, F. Retiere, H.G. Ritter, H.H. Wieman, S.E. Wurzel, E. Yamamoto, "Using an active pixel sensor in a vertex detector," accepted for publication in Proceedings of the Vertex 2003 Conference, Nuclear Instruments and Methods.

N.-H. Xuong, A.-C. Milazzo, P. LeBlanc, F. Duttweiler, J. Bouwer, S. Peltier, M. Ellisman, P. Denes, F. Bieser, H.S. Matis, H. Wieman, S. Kleinfelder, "First use of a highsensitivity active pixel sensor array as a detector for electron microscopy," Sensors and Camera Systems for Scientific, Industrial, and Digital Photography Applications V 5301, pp 242-249 (2004).

PBD-Cate LB03006 Microscopic Imaging in High-Throughput Screening for Crystals of the Bacterial Ribosome

Vila-Sanjurjo, A., Schuwrith, B.S., Hau,C.W. and Cate, J.H.D, "Structural basis for the control of translation initiation during stress," Nat. Struct. Molec. Biol., 11, 1054-1059 (2004).

The FY2004 funding also contributed to obtaining crystals of the intact bacterial ribosome that diffract $\mathrm{x}$-rays to atomic resolution. The solution of the structure of the bacterial ribosome at atomic resolution will be published in 2005 and 2006.

PBD-Marletta LB02041 Structure and Functional Characterization of Heme Protein Sensors

Pellicena, P., Karow, D.S., Marletta, M.A., Kuriyan, J.; "Crystal structure of an oxygenbinding heme domain related to soluble guanylate cyclases," Proc. Natl. Acad. Sci. USA 101(35):12854-12859 (2004).

Karow, D.S., Pan, D., Pellicena, P., Presley, A., Mathies, R.A. and Marletta, M.A.; "Spectroscopic characterization of the sGC-like heme domains from Vibrio cholerae and Thermoanaerobacter tengcongensis," Biochemistry 43: 10203-10211 (2004).

Gray, J.M., Karow, D.S., Lu, H., Chang, J.S., Marletta, M.A., Bargmann, C.I.; “Oxygen sensation and social feeding mediated by a C. elegans guanylate cyclase homologue," Nature 430: 317-322 (2004).

PBD-Yang LB02049 Conformation and Reaction Dynamics at the Single Molecule Level

L. P. Watkins and H. Yang, "Information Bounds and Optimal Analysis of Dynamic Single Molecule Measurements," Biophys. J. 86, 4015-4029 (2004).

A. Fu, C. M. Micheel, J. Cha, H. Chang, H. Yang, and A. P. Alivisatos, "Discrete Nanostructures of Quantum Dots/Au with DNA," J. Am. Chem. Soc., 126, 10832-10833 (2004). 
L. P. Watkins and H. Yang, "Detection of Intensity Change Points in Time-Resolved SingleMolecule Measurements," accepted by J. Phys. Chem. A (2005).

PD-Heeger LB04012 Designing a Novel Reactor Neutrino Experiment for Measuring the Mixing Angle Theta13

E. Abouzaid et al., "Report of the APS Neutrino Study Reactor Working Group, October 2004,” LBNL- 56599. Url: http://www.aps.org/neutrino/

K.M. Heeger, "Towards a Precision Measurement of $\theta_{13}$ with Reactor Neutrinos: Initiatives in the United States," Proceedings to 5th Workshop on "Neutrino Oscillations and their Origin" (NOON04), Odaiba, Tokyo, Japan, Feburary 11-15, 2004, September 2004, LBNL-56338.

K.M. Heeger, S.J. Freedman, R.W. Kadel, and K.-B. Luk, "Measuring $\theta_{13}$ with Reactor Neutrinos," Proceedings to 8th International Workshop on Topics in Astroparticle and Undergsround Physics (TAUP 2003), Seattle, Washington, 5-9 Sep 2003, July 2004, LBNL-55935.

C.T. Onishi. P. Dobson, S. Nakagawa, S. Glaser, D. Garlic, "Geologic Investigation of a Potential Site for a Next-Generation Reactor Neutrino Oscillation Experiment - Diablo Canyon, San Luis Obispo County, CA," LBNL-55692, June 2004.

D. Oshatz, " $\theta_{13}$ Neutrino Experiment at the Diablo Canyon Power Plant, LBNL Engineering Summary LBNL/PUB-5505, March 2004.

$\mathrm{K}$. Anderson et al. (International $\theta_{13}$ Working Group), "White Paper Report on Using Nuclear Reactors to Search for a Value of $\theta_{13}$," e-print arXive:hep-ex/0402041, 2004.

Cross-Div.-Geissler LB03035 Microscopic Theory of Protein Surface

P. Geissler and G. Crooks, "Changing patterns of amino acid sequence mutations", draft for possible submission to Proceedings of the National Academy of Science.

Cross-Div.-Harris et al. LB02010 Investigation of Charge Transfer in Organic Electronics Using Ultrafast Spectroscopy and Targeted Synthesis

S. Garrett-Roe, S. T. Shipman, P. Szymanski, M. L. Strader, A. Yang, and C. B. Harris, "Ultrafast electron dynamics at dielectric/metal interfaces: Intraband relaxation of image state electrons as friction," draft to be submitted to J. Phys. Chem. B.

S. Garrett-Roe, S. T. Shipman, P. Szymanski, M. L. Strader, A. Yang, and C. B. Harris, "Ultrafast electron dynamics at anthracene $/ \operatorname{Ag}(111)$ and xylene $/ \operatorname{Ag}(111)$ interfaces," draft to be submitted to Surface Science.

S. Garrett-Roe, S. T. Shipman, P. Szymanski, M. L. Strader, A. Yang, and C. B. Harris, "Ultrafast electron dynamics at fluorene oligomer/Ag(111) interfaces: Effects of surface structure and conjugation length on LUMO dynamics," draft to be submitted to $J$. Phys. Chem. B.

S. T. Shipman, P. Szymanski, S. Garrett-Roe, A. Yang, M. L. Strader, and C. B. Harris, "Ultrafast electron dynamics at $\mathrm{C} / \mathrm{Ag}(111)$ interfaces: A new method for measuring electronic transfer integrals," draft to be submitted to J. Phys. Chem. B.

A. Yang, S. T. Shipman, M. L. Strader, P. Szymanski, S. Garrett-Roe, and C. B. Harris, "Ultrafast dynamics of molecular excited states in ultrathin film PTCDA/Ag(111)," draft to be submitted to J. Phys. Chem. B. 
M. L. Strader, S. T. Shipman, S. Garrett-Roe, P. Szymanski, A. Yang, and C. B. Harris, "Ultrafast electron dynamics at DMSO/Ag(111): Two photon photoemission at electrochemically relevant interfaces," draft to be submitted to J. Phys. Chem. B.

S. T. Shipman, P. Szymanski, S. Garrett-Roe, A. Yang, M. L. Strader, and C. B. Harris, "Femtosecond two-photon photoemission studies of C/Ag(111)," accepted for publication in Abstr. Pap. Am. Chem. Soc.

M. L. Strader, S. T. Shipman, S. Garrett-Roe, P. Szymanski, A. Yang, and C. B. Harris, "Ultrafast electron dynamics of the image potential state at the DMSO/Ag(111) interface," accepted for publication in Abstr. Pap. Am. Chem. Soc.

A. Yang, S. T. Shipman, S. Garrett-Roe, M. L. Strader, P. Szymanski, and C. B. Harris, "Ultrafast dynamics of molecular excited states in ultrathin film PTCDA/Ag(111)," accepted for publication in Abstr. Pap. Am. Chem. Soc.

C. B. Harris, P. Szymanski, A. D. Miller, S. Garrett-Roe, S. T. Shipman, M. L. Strader, and A. Yang, "Dynamics of electrons at interfaces on the femtosecond timescale," accepted for publication in Abstr. Pap. Am. Chem. Soc.

Cross-Div.-Pines et al. LB03020 Ex-Situ and "Remote" Molecular Imaging and Spectroscopy

Thomas J. Lowery, Seth M. Rubin, E. Janette Ruiz, Alexander Pines, David E. Wemmer , "Design of a conformation-sensitive xenon-binding cavity in the ribose binding protein," Angewandte Chemie, 116, 2-4 (2004).

M. Spence, E.J. Ruiz, S. Rubin, T. Lowery, N. Winssinger, P. Schultz, D. Wemmer and A. Pines, "Development of a Functionalized Xenon Biosensor," JACS, 126, 15287-15294, (2004).

Daniel Topgaard, Rachel W. Martin, Dimitris Sakellariou, Carlos A. Meriles and Alexander Pines, "'Shim pulses' for NMR spectroscopy and imaging," PNAS 101 (51), 1757617581, (2004).

V. V. Yashchuk, J. Granwehr, D. F. Kimball, S. M. Rochester, A. H. Trabesinger, J. T. Urban, D. Budker, and A. Pines, "Hyperpolarized xenon nuclear spins detected by optical atomic magnetometry," Phys. Rev. Lett. 93(16), 160801 (2004).

R. McDermott, N. Kelso, S-K. Lee, M. Mössle, M. Mück, W. Myers, B. ten Haken, H.C. Seton, A.H. Trabesinger, A. Pines, and J. Clarke, "SQUID-Detected Magnetic Resonance Imaging in Microtesla Magnetic Fields," Journal of Low Temperature Physics, 135, 793821, (2004).

Dimitris Sakellariou, Carlos Meriles and Alexander Pines, "Advances in Ex-Situ Nuclear Magnetic Resonance," Comptes Rendus de l'Academie des Sciences Physique, 5, 337 347, (2004).

V. Demas, C. Meriles, D. Sakellariou, S. Han, J. Reimer, A. Pines, "3D Phase-Encoded Chemical Shift MRI in the Presence of Inhomogeneous Fields," Proc. of the Nat. Acad. Sci., 101 (24), 8845-8847, (2004).

R. McDermott, S.K. Lee, B. ten Haken, A.H. Trabesinger, A. Pines and J. Clarke, "Microtesla MRI with a superconducting quantum interference device," Proc. of the Nat. Acad. Sci, 101 (21), 7857-7861, (2004).

C.A. Meriles, D. Sakellariou, A. Moule, M. Goldman, T. Budinger and A. Pines, "Highresolution nuclear magnetic resonance of static samples by rotation of the magnetic field," J. Magnetic Resonance, 169, 13-18,(2004).

T.J. Lowery, S. Rubin, E. J. Ruiz, M. Spence, N. Wissinger, P. Schultz, A. Pines and D. Wemmer " "Applications of Laser-Polarized ${ }^{129}$ Xe NMR to Biomolecular Assays," Magnetic Resonance Imaging, 21, 1235-1239, (2003). 
D. Topgaard and A. Pines,"Self-diffusion Measurements with Chemical Shift Resolution in Inhomogeneous Magnetic Fields," J. Mag. Res., 168, 31-35, (2004).

Juliette A. Seeley, Song-I Han and Alexander Pines, "Remotely detected high-field MRI of porous samples," J. Mag Res., 167, 282-290, (2004).

D. Budker, D. F. Kimball, S. M. Rochester, J. T. Urban, "Alignment-to-orientation conversion and nuclear quadrupole resonance," Chem Phys. Lett., 378(3-4), 440-448 (2003).

\section{Manuscripts in press:}

S. Lee, M. Mößle, W. Myers,, N. Kelso, A.H. Trabesinger, J.T. Urban, D. Budker, A. Pines, "SQUID-Detected MRI at $132 \mu \mathrm{T}$ with $\mathrm{T}_{1}$ contrast weighted at $10 \mu \mathrm{T}-300 \mathrm{mT}$," in press Magnetic Resonance in Medicine.

R.W. Martin, R. Jachmann, D. Sakellariou, U.G. Nielsen, A. Pines, "High-resolution NMR spectroscopy of biological tissues using projected magic angle spinning," in press Magnetic Resonance in Medicine.

C.A. Meriles, D. Sakellariou, A.H. Trabesinger, V. Demas, A. Pines, "Zero to low-field MRI with Averaging of Concomitant Gradient Fields," accepted for publication in PNAS.

D. Sakellariou, C. Meriles, R.W. Martin and A. Pines, "NMR in rotating magnetic fields: Magic angle field spinning," in press Magnetic Resonance Imaging.

\section{Manuscripts submitted or in preparation:}

C. Meriles, D. Sakellariou, A. Moule, M. Goldman, T. Budinger and A. Pines, "HighResolution Nuclear Magnetic Resonance of Static Samples by Rotation of the Magnetic Field," submitted to Physical Review Letters.

J. Granwehr, J.T. Urban, A.H. Trabesinger, A. Pines, "External NMR Detection Using Hyperpolarized Xenon as a Sensor," in preparation.

C. Meriles, D. Sakellariou, A. Trabesinger, J. Clarke and A. Pines, "Tensor Field Magnetic Resonance Imaging," in preparation.

T.J. Lowery, M. Doucleff, E.J. Ruiz, S.M. Rubin, A. Pines and D. Wemmer, "Distinguishing Multiple Chemotaxis Y Protein Conformations with Laser-Polarized 129 Xe NMR," in preparation.

Vasiliki Demas, Carlos A. Meriles, Dimitris Sakellariou, Songi Han, Jeffrey Reimer and Alexander Pines, "Towards Ex-Situ: Chemical Shift MRI in a one-sided NMR probe," in preparation.

C. Meriles, D. Sakellariou, A. Trabesinger, V. Demas, J. Clarke, A. Pines, "Theory of Magnetic Resonance Imaging in Symmetrized Fields," in preparation.

Song-I Han, Kimberley Pierce, Alexander Pines, "Gas Flow Visualization by laser-Polarized ${ }^{129}$ Xe MRI," in preparation.

Josef Granwehr, Juliette Seeley, A. Pines, "Sensitivity Quantification of NMR Remote Detection," in preparation.

\section{Patents Issued:}

Method and Apparatus for High Resolution Ex Situ NMR Spectroscopy (2004) US 6,674,282 B2 (LBNL IB-1717)

Functionalized Active-Nucleus Complex Sensor (2003) US 6,652,883 (LBNL IB 1643)

Enhancement of NMR and MRI in the Presence of Hyperpolarized Noble Gases (2002) US 6,426,058 (LBNL IB-1168) licensed during period by Amersham Health (now GE Health) 
NMR/MRI with Hyperpolarized Gas and High Tc SQUID (2000) US 6,159,444 (LBNL IB1441)

\section{Patents Pending:}

Xenon Affinity Contrast Agents for high to zero-field MRI (2004) Disclosure.

"Shim Pulses" for NMR Spectroscopy and Imaging (2004) Disclosure (LBNL IB 2026P1)

Method and Apparatus for ultra-high resolution NMR Spectroscopy inside magnets (2004) Disclosure (LBNL IB-2026P)

SQUID-detected NMR and MRI at Ultralow Fields (2003) Application filed (LBNL IB1729)

Remote NMR Detection of Laser-Polarized Gases (2002) Provisional, PCT and application filed (LBNL IB-1771)

Method for Detecting Macromolecular Conformational Change and Binding Information (2002) Application filed (LBNL IB-1718)

Amplified Xe NMR/MRI by remote optical detection (2001) Provisional filed (LBNL IB1715) 
